\title{
Composites and Categories of Euclidean Jordan Algebras
}

\author{
Howard Barnum ${ }^{1,2,3}$, Matthew A. Graydon ${ }^{4,5}$, and Alexander Wilce ${ }^{6}$ \\ ${ }^{1}$ Riemann Center for Geometry and Physics, Institute for Theoretical Physics, Leibniz Universität Hannover \\ ${ }^{2}$ University of New Mexico \\ ${ }^{3}$ Currently unaffiliated hnbarnum@aol.com \\ ${ }^{4}$ Department of Applied Mathematics, University of Waterloo \\ ${ }^{5}$ Institute for Quantum Computing, University of Waterloo m3graydo@uwaterloo.ca \\ ${ }^{6}$ Department of Mathematical Sciences, Susquehanna University wilce@susqu.edu
}

We consider possible non-signaling composites of probabilistic models based on euclidean Jordan algebras (EJAs), satisfying some reasonable additional constraints motivated by the desire to construct dagger-compact categories of such models. We show that no such composite has the exceptional Jordan algebra as a direct summand, nor does any such composite exist if one factor has an exceptional summand, unless the other factor is a direct sum of one-dimensional Jordan algebras (representing essentially a classical system). Moreover, we show that any composite of simple, nonexceptional EJAs is a direct summand of their universal tensor product, sharply limiting the possibilities.

These results warrant our focussing on concrete Jordan algebras of hermitian matrices, i.e., euclidean Jordan algebras with a preferred embedding in a complex matrix algebra. We show that these can be organized in a natural way as a symmetric monoidal category, albeit one that is not compact closed. We then construct a related category InvQM of embedded euclidean Jordan algebras, having fewer objects but more morphisms, that is not only compact closed but dagger-compact. This category unifies finite-dimensional real, complex and quaternionic mixed-state quantum mechanics, except that the composite of two complex quantum systems comes with an extra classical bit.

Our notion of composite requires neither tomographic locality, nor preservation of purity under tensor product. The categories we construct include examples in which both of these conditions fail. In such cases, the information capacity (the maximum number of mutually distinguishable states) of a composite is greater than the product of the capacities of its constituents. 


\section{Introduction}

Formally real Jordan algebras were first proposed as models of quantum systems by P. Jordan in 1933 [40]. Abstractly, a Jordan algebra is a real vector space $A$ equipped with a commutative bilinear product $\bullet$ satisfying the Jordan identity $a^{2} \bullet(a \bullet b)=a \bullet\left(a^{2} \bullet b\right)$ for all $a, b \in A$ (where $a^{2}=a \bullet a$ ). We also assume that $A$ has a unit element, which we denote by $u_{A}$. $A$ is formally real if, for $a_{1}, \ldots, a_{n} \in A, \sum_{i=1}^{n} a_{i}^{2}=0$ only when $a_{i}=0$ for all $i$. If $A$ is finite-dimensional, this is equivalent to $A$ 's being euclidean, meaning that it carries an inner product such that $\langle a \bullet b, c\rangle=\langle a, b \bullet c\rangle$ for all $a, b, c \in A$. It will be convenient in what follows to compress the phrase euclidean Jordan algebra, which will occur very frequently, to the acronym EJA.

The standard example is the space $\mathcal{L}(\mathbf{H})$ of self-adjoint operators on a finitedimensional Hilbert space $\mathbf{H}$, with $a \bullet b=(a b+b a) / 2$, and with $\langle a, b\rangle=\operatorname{Tr}(a b)$. In 1934, Jordan, von Neumann and Wigner [43] showed that all finite-dimensional formally real - equivalently, euclidean - Jordan algebras are direct sums of irreducible, or simple, such algebras, and that the latter are of just five kinds: self-adjoint parts of real, complex or quaternionic matrix algebras (which we can regard as real, complex or quaternionic quantum systems) spin factors (which are analogues of qubits in which the "Bloch sphere" can have arbitrary finite dimension), and the exceptional Jordan algebra of $3 \times 3$ self-adjoint octonionic matrices.

A reasonable objection is that the physical meaning of the Jordan product is obscure. (Indeed, it is not obvious why the observables of a physical system should carry any physically meaningful bilinear product at all.) Happily, there are alternative characterizations of euclidean Jordan algebras in terms of ordered vector spaces and related concepts having readier physical, probabilistic, or operational interpretations. The Koecher-Vinberg Theorem ([45, 62]; see also [44], [26], Chapter III or [56], Chapter I, §8) identifies euclidean Jordan algebras with finite-dimensional ordered vector spaces having homogeneous, self-dual cones; work of Alfsen and Shultz [2, 4] characterizes EJAs in terms of certain projections associated with closed faces of the positive cone. ${ }^{1}$ Exploiting these results, several recent papers [15, 14, 53, 63, 64, 66, 65] have shown that physically reasonable postulates force a finite-dimensional physical system to have the structure of a euclidean Jordan algebra. To this extent, euclidean Jordan algebras are a natural class of models for physical systems.

A physical theory, however, is more than a collection of models of physical systems. It must also describe how systems change and how they interact. It is natural, therefore, to represent a physical theory as a category, in which objects represent physical systems and morphisms represent physical processes. To accommodate composite systems, one wants the category to be monoidal, i.e., to be equipped with an associative "tensor product". This point of view has been developed very fruitfully in $[1,8]$ and elsewhere, where it is shown that many features of finite-dimensional quantum mechanics can be

\footnotetext{
${ }^{1}$ Alfsen and Shultz' results apply, more generally, to JB-algebras, which in the context of finite dimension are the formally real Jordan algebras.
}

Accepted in 〈 xuantum 2020-10-07, click title to verify. Published under CC-BY 4.0. 
recovered if the category in question is compact closed, or, better still, dagger-compact (terms we explain in Section 5).

In this paper, building on work of Hanche-Olsen [30] and Jamjoom [38] on tensor products of JC-algebras, we classify the possible composites of euclidean Jordanalgebraic systems, subject to a standard "no-signaling" condition and a few reasonable additional constraints. In particular, we show that no such composite exists if either factor is exceptional, unless the other factor is a direct sum of trivial (i.e. 1-dimensional) Jordan algebras. Furthermore, we show that a composite of simple, nontrivial Jordan algebras is always a special Jordan algebra, and, indeed, a direct summand of the universal tensor product defined by Hanche-Olsen

Restricting attention further to Jordan algebras corresponding to real, complex and quaternionic quantum systems - equivalently, self-adjoint parts of real, complex and quaternionic matrix algebras - we then identify two different monoidal sub-categories extending the category of finite-dimensional complex matrix algebras and CP maps. One of these, which we call RSE, unifies real, complex and quaternionic quantum mechanics, but lacks certain desirable features. In particular, in this category, states are not represented by morphisms; hence, the category is far from being compact closed. The other category, which we call InvQM, and which is compact closed, also embraces real, complex and quaternionic quantum systems and processes (CP maps), except that its rule for composing standard complex quantum systems yields an extra classical bit.

These results, combined with the those of (any of) the papers cited above, in which a euclidean Jordan structure emerges from information-theoretically, physically or operationally natural assumptions, lend support to the idea of unified quantum theory that embraces real, complex and quaternionic quantum systems, and permits the formation of composites of these. Consistent with the results of [17], the composites that arise in these constructions do not in general have the property of "tomographic locality": states on the composite of two Jordan-algebraic systems are not, in general, determined by the joint probabilities they assign to measurement outcomes associated with the two component systems. Equivalently, the Jordan algebra $A B$ corresponding to a composite of two formally real Jordan-algebraic systems $A$ and $B$, will generally be larger than the algebraic (i.e., vector-space) tensor product $A \otimes B$.

Remark: A related proposal is advanced by Baez [7], who points out that one can regard real and quaternionic quantum systems as pairs $(\mathbf{H}, J)$, where $\mathbf{H}$ is a complex Hilbert space and $J$ is an anti-unitary satisfying $J^{2}=\mathbf{1}$ (the real case) or $J^{2}=\mathbf{- 1}$ (the quaternionic case). Such pairs can be organized into a dagger-compact symmetric monoidal category, taking morphisms $\left(\mathbf{H}_{1}, J_{1}\right) \rightarrow\left(\mathbf{H}_{2}, J_{2}\right)$ to be linear mappings intertwining $J_{1}$ and $J_{2}$, and $\left(\mathbf{H}_{1}, J_{1}\right) \otimes\left(\mathbf{H}_{2}, J_{2}\right)=\left(\mathbf{H}_{1} \otimes \mathbf{H}_{2}, J_{1} \otimes J_{2}\right)$. This provides a unification of real and quaternionic quantum mechanics at the level of pure states and linear mappings between the relevant Hilbert spaces, whereas our approach takes in quantum systems over all three of the associative division algebras $\mathbb{R}, \mathbb{C}$ and $\mathbb{H}$, at the level of mixed states, observables and completely positive maps. While the precise connection between Baez' approach and ours is not yet entirely clear, it seems to us 
likely that an application of Selinger's CPM construction [57] to Baez' category will yield a category of the type we consider here.

Our results rest on a mixture of standard facts about ordered vector spaces, the order structure and the representation theory of euclidean Jordan algebras, and the pioneering work of Hanche-Olsen [30] on universal representations and tensor products of JC-algebras. Since much of this will be unfamiliar to many readers, we have included a good amount of purely expository material. Section 2 provides background on order unit spaces and their interpretation as general probabilistic models, including a fairly general notion of composite for such model. This material will be more familiar to many readers, but some of our notation and terminology, and some notions specific to our present purposes, may not be. Section 3 collects background material on euclidean Jordan algebras, their universal representations, and Hanche-Olsen's universal tensor product.

The balance of the paper is organized as follows. In Section 4, we introduce a general definition for the composite of two euclidean Jordan-algebraic probabilistic models, and establish some basic properties of any such composite. Along the way, we see that the composite of two simple, nontrivial EJAs must be embeddable in a complex matrix algebra, i.e. it must be special (Theorem 4.12). From this it follows that no simple, nontrivial EJA has any composite with an exceptional EJA (Corollary 4.14), and that if $A$ and $B$ are simple, special EJAs, then any composite of $A$ and $B$ must be an ideal - that is, a direct summand - in their universal tensor product (Theorem 4.15).

These results warrant our focusing on special EJAs. Section 5 develops a canonical, and naturally associative, tensor product of embedded EJAs, that is, pairs $\left(A, \mathbf{M}_{A}\right)$ where $\mathbf{M}_{A}$ is a finite-dimensional complex *-algebra and $A$ is a Jordan subalgebra of the self-adjoint part of $\mathbf{M}_{A}$. (The universal tensor product is the special case in which $\mathbf{M}_{A}$ and $\mathbf{M}_{B}$ are the universal complex enveloping algebras of $A$ and $B$.) In Section 6 we introduce a class of mappings we call completely Jordan-preserving and use these to construct symmetric monoidal categories of embedded EJAs, some of which we then show are compact closed or, indeed, dagger-compact. Section 7 concludes with further discussion of these categories and their physical and information-processing significance. To avoid obstructing the flow of the main arguments, we have removed some technical details to a series of appendices.

Acknowledgments Some of our results have previously been announced, without proof, in [13]. ${ }^{2} \quad \mathrm{HB}$ and AW wish to thank C. M. Edwards for introducing them to the paper [30] of Hanche-Olsen. HB and MG thank Cozmin Ududec for valuable discussions. AW was supported by a grant from the FQXi foundation (FQXi-RFP3-1348). This research was supported in part by Perimeter Institute for Theoretical Physics. Research at Perimeter Institute is supported by the Government of Canada through the Department of Innovation, Science and Economic Development Canada and by the Province of Ontario through the Ministry of Research, Innovation and Science.

\footnotetext{
${ }^{2}$ There, it is erroneously claimed that RSE is compact closed. This error is corrected in Section 6 of the present paper; see especially Examples 6.3 and 6.11
}

Accepted in 〈 Xuantum 2020-10-07, click title to verify. Published under CC-BY 4.0. 


\section{Ordered vector spaces and probabilistic models}

In this section and the next, we present enough background material to make this paper reasonably self-contained. This section summarizes basic information about ordered vector spaces and the "convex operational" (or "generalized probabilistic theories") framework for discussing probabilistic physical theories.

A good general reference for ordered vector spaces is Chapter 1 of [3] (or the summary in Appendix A of [4]). The use of ordered vector spaces with an order unit as probabilistic models goes back at least to the work of Ludwig [46, 47]; see also $[24,25,35]$. The more recent literature in this tradition (for a survey of which, see [18]) has focussed on finite-dimensional quantum theory, and accordingly makes use only of finite-dimensional ordered spaces. This will also be the case in this paper. Hence, in order to avoid constant repetition of the qualifier, we adopt the convention that all vector spaces are finite dimensional unless otherwise indicated. All topological statements concerning such a space, for example references to the interior of a subset, should be understood as referring to its unique linear topology.

\subsection{Ordered vector spaces}

Let $A$ be a real vector space. A (convex, pointed) cone in $A$ is a convex set $K \subseteq A$ such that $a \in K$ implies $t a \in K$ for all $t \in \mathbb{R}_{+}$, and $K \cap-K=\{0\}$. A cone $K$ is generating iff it spans $A$, i.e., if every $a \in A$ can be expressed as a difference of elements of $K$. Any cone (generating or not) induces a partial ordering of $A$, given by $a \leq b$ iff $b-a \in K$; this is translation-invariant, i.e, $a \leq b$ implies $a+c \leq b+c$ for all $a, b, c \in A$, and homogeneous, i.e., $a \leq b$ implies $t a \leq t b$ for all $t \in \mathbb{R}_{+}$. Conversely, such an ordering determines a cone, namely $K=\{a \mid a \geq 0\}$. Accordingly, an ordered vector space is a real vector space $A$ equipped with a designated positive cone cone $A_{+}$. It is common to assume, and we shall assume here, that $A_{+}$is closed and generating.

If $A$ and $B$ are ordered vector spaces, a linear mapping $f: A \rightarrow B$ is positive iff $f\left(A_{+}\right) \subseteq B_{+}$. If $f$ is bijective and $f\left(A_{+}\right)=B_{+}$, then $f^{-1}\left(B_{+}\right)=A_{+}$, so that $f^{-1}$ is also positive. In this case, we say that $f$ is an order isomorphism. An order automorphism of $A$ is an order isomorphism from $A$ to itself.

Denoting the dual space of $A$ by $A^{*}$, the dual cone, $A_{+}^{*}$, is the set of positive linear functionals on $A$. Since we are assuming that $A_{+}$is generating, it is easy to see that $A_{+}^{*} \cap-A_{+}^{*}=\{0\}$. In our finite-dimensional setting, $A_{+}^{*}$ is also generating. Note that if $B$ is another ordered vector space and $\phi: A \rightarrow B$ is a positive linear mapping, then the dual mapping $\phi^{*}: B^{*} \rightarrow A^{*}$ is also positive.

\subsection{Order units and probabilistic models}

An order unit in an ordered vector space $A$ is an element $u \in A_{+}$such that, for all $a \in A, a \leq t u$ for some $t \in \mathbb{R}_{+}$. In finite dimensions, this is equivalent to $u$ belonging 
to the interior of $A_{+}$(cf. [6], Theorem 2.8). An order unit space is a pair $(A, u)$ where $A$ is an ordered vector space and $u$ is a designated order unit.

An order unit space provides the machinery to discuss probabilistic concepts. A state on $(A, u)$ is a positive linear functional $\alpha \in A^{*}$ with $\alpha(u)=1$. An effect is an element $a \in A_{+}$with $a \leq u$. If $\alpha$ is a state and $a$ is an effect, we have $0 \leq \alpha(a) \leq 1$ : we interpret this as the probability of the given effect on the given state.

A discrete observable on $A$ with values $\lambda \in \Lambda$ is represented by an indexed family $\left\{a_{\lambda} \mid \lambda \in \Lambda\right\}$ of effects summing to $u$, the effect $a_{\lambda}$ representing the event of obtaining value $\lambda$ in a measurement of the observable. Thus, if $\alpha$ is a state, $\lambda \mapsto \alpha\left(a_{\lambda}\right)$ gives a probability weight on $\Lambda$. (One can extend this discussion to include more general observables by considering effect-valued measures (cf. [25]), but we will not need this extra generality here.)

We denote the set of all states of $A$ by $\Omega$; the set of all effects - the interval between 0 and $u$ - is denoted $[0, u]$. In our present finite-dimensional setting, both are compact convex sets. Extreme points of $\Omega$ are called pure states.

\section{Examples}

(1) Discrete Classical Probability Theory: If $S$ is a finite set, regarded as the outcome space of some classical experiment, let $A(S)=\mathbb{R}^{S}$, ordered pointwise. Elements of $A(S)_{+}$are then non-negative random variables, and effects are random variables with values between 0 and 1 . We turn this into an order unit space by taking $u \in A(S)_{+}$to be the constant function with value 1 . It is then easy to show that normalized states on $A(S)$ correspond exactly to probability weights on $S$; discrete observables correspond in a natural way to discrete "fuzzy" random variables. Extreme effects, i.e, extreme points of $[0, u]$, are easily seen to be characteristic functions of subsets of $S$; hence, an observable $\left\{a_{\lambda}\right\}$ with $a_{\lambda}$ extreme for each $\lambda$, corresponds to an ordinary "sharp" random variable.

(2) Discrete Quantum Probability Theory: If $\mathbf{H}$ is a finite-dimensional Hilbert space, let $A(\mathbf{H})=\mathcal{L}(\mathbf{H})$, the space of self-adjoint operators on $\mathbf{H}$, ordered by the cone of positive semidefinite operators; let $u=\mathbf{1}$, the identity operator on $\mathbf{H}$. Then each normalized state $\alpha$ has the form $\alpha(a)=\operatorname{Tr}(\rho a)$ where $\rho$ is a density operator, and, conversely, every density operator determines in this way a normalized state,. Observables correspond to discrete POVMs; thus, we recover orthodox finite-dimensional quantum probability theory.

(3) A class of examples that embraces both (1) and (2) is the following. Let $\mathbf{M}$ be a unital complex *-algebra; define $\mathbf{M}_{+}$to consist of all $a \in \mathbf{M}$ with $a=b^{*} b$ for some $b \in \mathbf{M}$ : then $\left(\mathbf{M}_{s a}, \mathbf{M}_{+}\right)$is an ordered vector space, in which the unit element of $\mathbf{M}$ serves as an order unit. If $\mathbf{M}$ is finite-dimensional and commutative, one essentially recovers example (1); if $\mathbf{M}$ is the algebra $M_{n}(\mathbb{C})$ of $n \times n$ complex matrices, one essentially recovers example (2). More generally, if $\mathbf{M}$ is finite-dimensional, Wedderburn's theorem tells us that $\mathbf{M}$ is a direct sum of matrix algebras, so one has finite-dimensional quantum theory with superselection rules (classical discrete probability theory being the special case in which all superselection sectors - that is, direct summands - are 
one-dimensional).

One might wish to privilege certain states and/or certain effects of a probabilistic model as being "physically possible". One way of doing so is to consider ordered subspaces $V \leq A^{*}$ and $E \leq A$, with $u \in E$ : this picks out the set of states $\alpha \in V \cap A_{+}^{*}$ and the set of effects $a \in E \cap A_{+}, a \leq u$. The pair $(E, V)$ then serves as a probabilistic model for a system having these allowed states and effects. However, in the three examples above, and in those that concern us in the rest of this paper, it is always possible to regard all states in $A^{*}$, and all effects in $A$, as allowed. Henceforth, then, when we speak of a probabilistic model - or, more briefly, a model - we simply mean an order unit space $(A, u)$. It will be convenient to adopt the shorthand $A$ for such a pair, writing $u_{A}$ for the order unit where necessary.

Processes, Symmetries and Dynamics By a process affecting a system represented by a probabilistic model $A$, we mean a positive linear mapping $\phi: A \rightarrow A$, subject to the condition that $\phi\left(u_{A}\right) \leq u_{A}$. The probability of observing an effect $a$ after the system has been prepared in a state $\alpha$ and then subjected to a process $\phi$ is $\alpha(\phi(a))$. One can regard $\alpha(\phi(u))$ as the probability that the system is not destroyed by the process. We can, of course, replace $\phi: A \rightarrow A$ with the adjoint mapping $\phi^{*}: A^{*} \rightarrow A^{*}$ given by $\phi^{*}(\alpha)=\alpha \circ \phi$, so as to think of a process as a mapping from states to possibly sub-normalized states. Thus, we can view processes either as acting on effects (the "Heisenberg picture"), or on states (the "Schrödinger picture").

Any non-zero positive linear mapping $\phi: A \rightarrow A$ is a non-negative scalar multiple of a process in the above sense: since $\Omega(A)$ is compact, the function $\alpha \mapsto \alpha\left(\phi\left(u_{A}\right)\right)$ attains a maximum value $m>0$ on $\Omega(A) ; m^{-1} \phi$ is then a process. For this reason, we make little further distinction here between processes and positive mappings. In particular, if $\phi$ is an order automorphism of $A$, then both $\phi$ and $\phi^{-1}$ are scalar multiples of processes in the above sense: each of these processes "undoes" the other, up to normalization, i.e., with nonzero probability. A process that can be reversed with probability one is represented by an order-automorphism $\phi$ such that $\phi\left(u_{A}\right)=u_{A}$, in which case $\phi^{*}$ takes normalized states to normalized states. Such an order-automorphism is called a symmetry of $A$.

We denote the group of all order-automorphisms of $A$ by $\operatorname{Aut}(A) .^{3}$ This is a Lie group (see e.g. [34], pp. 182-183); its connected identity component (consisting of those processes that can be obtained by continuously deforming the identity map) is denoted $\operatorname{Aut}_{0}(A)$. A possible (probabilistically) reversible dynamics for a system modelled by $A$ is a homomorphism $t \mapsto \phi_{t}$ from $(\mathbb{R},+)$ to $\operatorname{Aut}(A)$, i.e., a one-parameter subgroup of $\operatorname{Aut}(A)$.

One might wish to privilege certain processes as reflecting physically possible motions or evolutions of the system. In that case, one might add to the basic data $(A, u)$ a preferred group $G(A) \leq \operatorname{Aut}(A)$ of order automorphisms. We refer to such a structure

\footnotetext{
${ }^{3}$ Here our usage diverges from that of [4] and [26], who use $\operatorname{Aut}(A)$ to denote the group of Jordan automorphisms of a Jordan algebra $A$.
} 
as a dynamical probabilistic model, since the choice of $G(A)$ constrains the permitted probabilistically reversible dynamics of the model. ${ }^{4}$

When $\operatorname{Aut}(A)$ acts transitively on the interior of $A_{+}$, the cone $A_{+}$(or the ordered space $A$ ) is said to be homogeneous. The positive cone of a euclidean Jordan algebra is always homogeneous, as we will see below. ${ }^{5}$

Self-Duality An inner product $\langle\mid\rangle$ on an ordered vector space $A$ is positive iff the associated mapping $A \rightarrow A^{*}, a \mapsto\langle a|$, is positive, i.e,. if $\langle a, b\rangle \geq 0$ for all $a, b \in A_{+}$. We say that $\langle\mid\rangle$ is self-dualizing if $a \mapsto\langle a|$ maps $A_{+}$onto $A_{+}^{*}$, so that $a \in A_{+}$if and only if $\langle a, b\rangle \geq 0$ for all $b \in B$. We say that $A$ (or its positive cone) is self-dual if $A$ admits a self-dualizing inner product. In this case, we can represent states of $A$ internally: if $\alpha \in A_{+}^{*}$ with $\alpha(u)=1$, there is a unique $a \in A_{+}$with $\langle a \mid b\rangle=\alpha(b)$ for all $b \in A_{+}$. Conversely, if $a \in A_{+}$with $\langle a, u\rangle=1$, then $\langle a|$ is a state. We will also use the notation $\langle$,$\rangle for an inner product.$

The probabilistic models associated with classical and quantum systems, as discussed above, are self-dual. Indeed, in non-relativistic quantum theory, where $A=$ $\mathcal{L}(\mathbf{H})$, the standard trace inner product $\langle a, b\rangle=\operatorname{Tr}(a b)$ is self-dualizing. Here it is usual to identify states internally, i.e., as density operators.

If $A$ and $B$ are both self-dual and $\phi: A \rightarrow B$ is a positive linear mapping, we can use self-dualizing inner products on $A$ and $B$ to represent the mapping $\phi^{*}: B^{*} \rightarrow A^{*}$ as a positive linear mapping $\phi^{\dagger}: B \rightarrow A$, setting $\left\langle a, \phi^{\dagger}(b)\right\rangle=\langle\phi(a), b\rangle$ for all $a \in A$ and $b \in B$. If $\phi: A \rightarrow A$ is an order-automorphism, then so is $\phi^{\dagger}$.

\subsection{Composites of probabilistic models}

If $A$ and $B$ are probabilistic models of two physical systems, one may want to construct a model of the pair of systems considered together. In quantum mechanics, where $A=$ $\mathcal{L}\left(\mathbf{H}_{1}\right)$ and $B=\mathcal{L}\left(\mathbf{H}_{2}\right)$, one would form the model $A B=\mathcal{L}\left(\mathbf{H}_{1} \otimes \mathbf{H}_{2}\right)$ associated with the tensor product of the two Hilbert spaces. In the framework of general probabilistic models, there is no such canonical choice for a model of a composite system. However, one can at least say what one means by a composite of two probabilistic models: at a minimum, one should be able to perform measurements on the two systems separately, and compare the results. More formally, there should be a mapping $\pi: A \times B \rightarrow A B$ taking each pair of effects $(a, b) \in A \times B$ to an effect $\pi(a, b) \in A B$. One would like

\footnotetext{
${ }^{4} \mathrm{~A}$ more general definition of a dynamical model would require only that the set of possible evolutions form a semigroup of positive maps. This level of generality will emerge naturally later in this paper, when we consider categories of systems. The definition above is sufficient for our immediate purposes.

${ }^{5}$ Although we do not need this fact, we note that for a homogeneous cone in an ordered vector space $A$, the connected identity component $\operatorname{Aut}_{o}(A)$ of the $\operatorname{Aut}(A)$ also acts transitively on the interior of $A_{+}$; see e.g. [26], pp. 5-6.
} 
this to be non-signaling, meaning that the probability of obtaining a particular effect on one of the component systems in a state $\omega \in \Omega(A B)$ should be independent of what observable is measured on the other system. One can show that this is equivalent to $\pi$ 's being bilinear, with $\pi\left(u_{A}, u_{B}\right)=u_{A B}$ [18]. Finally, one would like to be able to prepare $A$ and $B$ separately in arbitrary states. Summarizing:

Definition 2.1. A (non-signaling) composite of probabilistic models $A$ and $B$, is a pair $(A B, \pi)$ where $A B$ is a probabilistic model, and $\pi: A \times B \rightarrow A B$ is a bilinear mapping such that

(a) $\pi(a, b) \in(A B)_{+}$for all $a \in A_{+}$and $b \in B_{+}$;

(b) $\pi\left(u_{A}, u_{B}\right)=u_{A B}$;

(c) For every pair of states $\alpha \in \Omega(A)$ and $\beta \in \Omega(B)$, there exists a state $\gamma \in \Omega(A B)$ such that for every pair of effects $a \in A$ and $b \in B, \gamma(\pi(a, b))=\alpha(a) \beta(b) .{ }^{6}$

Since $\pi$ is bilinear, it extends uniquely to a linear mapping $A \otimes B \rightarrow A B$. In what follows, we abuse notation slightly to denote this unique extension also by $\pi$, so that, for instance, $\pi(a \otimes b)=\pi(a, b)$ for $a \in A, b \in B$.

Lemma 2.2. $\pi$ is injective.

Proof: If $\pi(T)=0$ for some $T \in A \otimes B$, then for each pair of states $\alpha \in \Omega(A), \beta \in \Omega(B)$, we have by (c) of Definition 2.1 a state $\gamma \in \Omega(A B)$ with $(\alpha \otimes \beta)(T)=\gamma(\pi(T))=0$. But then $T=0$ since product states span $(A \otimes B)^{*}$.

This warrants our treating $A \otimes B$ as a subspace of $A B$ and writing $a \otimes b$ for $\pi(a, b)$. Note that if $\omega$ is a state on $A B$, then $\pi^{*}(\omega):=\omega \circ \pi$ defines a joint probability assignment on effects of $A$ and $B$ :

$$
\pi^{*}(\omega)(a, b)=\omega(a \otimes b) .
$$

This gives us marginal states $\omega_{A}=\omega\left(u_{A} \otimes-\right)$ and $\omega_{B}=\omega\left(-\otimes u_{B}\right)$. Where these are non-zero, we can also define conditional states $\omega_{1 \mid b}(a):=\omega(a \otimes b) / \omega_{B}(b)$ and $\omega_{2 \mid a}(b)=$ $\omega(a \otimes b) / \omega_{A}(a) .^{7}$

When the mapping $\pi: A \otimes B \rightarrow A B$ is also surjective, we can identify $A B$ with $A \otimes B$. The joint probability assigment $\pi^{*}(\omega)$ then completely determines $\omega$, so that states on $A B$ are such joint probability assignments. In this case, we say that $A B$ is locally tomographic, since states of $A B$ can be determined by comparing the results of "local" measurements, i.e., measurements carried out on $A$ and $B$ alone. In finite dimensions, both classical and complex quantum-mechanical composites have this feature, while composites of real quantum systems are not locally tomographic [5, 33].

\footnotetext{
${ }^{6} \gamma$ is unique if $A B$ is locally tomographic.

${ }^{7}$ In the context of a more general definition of probabilistic models, in which the cone generated by allowed states might not be the full dual cone $A_{+}^{*}$, we would need to modify this definition to enforce that these conditional states belong to the allowed state-space. See [18] for details.
} 
When dealing with dynamical probabilistic models, one needs to supplement conditions (a), (b) and (c) with the further condition that it should be possible for $A$ and $B$ to evolve independently within the composite $A B$. That is:

Definition 2.3. A dynamical composite of dynamical probabilistic models $A$ and $B$ is a composite $A B$, in the sense of Definition 2.1, plus a homomorphism

$$
\otimes: G(A) \times G(B) \rightarrow G(A B)
$$

such that $(g \otimes h)(a \otimes b)=g a \otimes h b$ for all $g \in G(A), h \in G(B), a \in A$ and $b \in B$.

(Note that since $A B$ may be larger than the algebraic tensor product $A \otimes B$, the order automorphism $(g \otimes h)$ need not be uniquely determined by the aforementioned condition.)

\subsection{Probabilistic theories as categories}

A physical theory is more than a collection of models. At a minimum, one also needs the means to describe interactions between physical systems. A natural way of accomplishing this is to treat physical theories as categories, in which objects represent physical systems, and morphisms represent processes. In the setting of this paper, then, it's natural to regard a probabilistic theory as a category $\mathcal{C}$ in which objects are probabilistic models, i.e., order unit spaces, and in which morphisms give rise to positive linear mappings between these.

The reason for this phrasing - morphisms giving rise to, as opposed to simply being, positive linear mappings - is to allow for the possibility that two abstract processes that behave the same way on effects of their source system, may differ in other ways - even in detectable ways, such as their effect on composite systems of which the source and target systems are components. If distinct morphisms between the same two objects always induce distinct positive maps, we say the category, and the set of morphisms, has local process tomography ${ }^{8}$. Notice that invertible morphisms $A \rightarrow A$ that preserve the order unit then induce processes in the sense given above, so that every model $A \in \mathcal{C}$ carries a distinguished group of reversible processes: models in $\mathcal{C}$, in other words, are automatically dynamical models.

In order to allow for the formation of composite systems, it is natural to ask that $\mathcal{C}$ be a symmetric monoidal category. That is, we wish to equip $\mathcal{C}$ with a bifunctorial product $\otimes: \mathcal{C} \times \mathcal{C} \rightarrow \mathcal{C}$ that is naturally associative and commutative, and for which there is a unit object $I$ with $I \otimes A \simeq A \simeq A \otimes I$ for objects $A \in \mathcal{C}$. Of course, we want to take $I=\mathbb{R}$. Moreover, for objects $A, B \in \mathcal{C}$, we want $A \otimes B$ to be a composite in the

\footnotetext{
${ }^{8}$ One way to make this more precise is to require that $\mathcal{C}$ contain $\mathbb{R}$, ordered as usual and with order unit 1 , and that $\mathcal{C}(I, A)$ be the cone of positive linear maps $\mathbb{R} \rightarrow A$, so that $\mathcal{C}(I, A) \simeq A_{+}$. Any morphism $\phi \in \mathcal{C}(A, B)$ then gives rise to a mapping $\widehat{\phi}: \mathcal{C}(I, A) \rightarrow \mathcal{C}(I, B)$ by $\widehat{\phi}(a)=\phi \circ a$ for every $a \in \mathcal{C}(I, A)$; this extends to a positive linear mapping $A \rightarrow B$. We shall not pursue this further here; see [16] for more on these lines.
} 
sense of Definitions 2.1 and 2.3 above. In fact, though, every part of those definitions simply follows from the monoidality of $\mathcal{C}$, except for part (b) of 2.1; we must add "by hand" the requirement that $u_{A} \otimes u_{B}=u_{A \otimes B}$. The category will also pick out, for each object $A$, a preferred group $G(A)$, namely, the group of invertible morphisms in $\mathcal{C}(A, A)$. The monoidal structure then picks out, for $g \in g(A)$ and $h \in G(B)$, a preferred $g \otimes h \in G(A B)$. The bifunctoriality of $\otimes$ guarantees that this will satisfy condition (b) of Definition 2.3; it will also satisfy condition (a) as long as $\mathcal{C}(I, A)=\mathcal{L}_{+}(\mathbb{R}, \mathcal{A}) \simeq \mathcal{A}_{+}$.

\section{Background on Euclidean Jordan algebras}

In this section, we summarize the essential background information on euclidean Jordan algebras and their universal tensor products that will be used in the sequel. General references for this material are the monographs [4] of Alfsen and Shultz and [26] of Faraut and Koranyi and [31] of Hanche-Olsen and Størmer, plus the paper [30] of Hanche-Olsen.

\subsection{Euclidean Jordan algebras}

As we have already mentioned, a euclidean Jordan algebra (hereafter: EJA) is a finitedimensional commutative (but not necessarily associative) real algebra $(A, \bullet)$ with a multiplicative unit element $u$, satisfying the Jordan identity $a^{2} \bullet(a \bullet b)=a \bullet\left(a^{2} \bullet b\right)$ for all $a, b \in A$, and equipped with an inner product satisfying $\langle a \bullet b, c\rangle=\langle b, a \bullet c\rangle$ for all $a, b, c \in A$. Obviously, any commutative, associative real algebra provides an example, but not a very interesting one from the algebraic point of view. The basic nonassociative example is the self-adjoint part $M_{s a}$ of a complex matrix algebra $M$, with $a \bullet b=(a b+b a) / 2$ and with $\langle a, b\rangle=\operatorname{Tr}(a b)$. A Jordan subalgebra of a Jordan algebra $A$ is a subspace $B$ of $A$ that is closed under inclusion of Jordan products, and hence is a Jordan algebra with Jordan product given by the restriction of $A$ 's. Any Jordan subalgebra of an EJA is also an EJA. Since real and quaternionic matrix algebras have representations as subalgebras of complex matrix algebras, their self-adjoint parts are EJAs. So, too, is the spin factor $V_{n}=\mathbb{R} \times \mathbb{R}^{n}$, with an inner product given by the usual vector dot product, and with a Jordan product given by

$$
(t, x) \bullet(s, y)=(t s+\langle x, y\rangle, t y+s x)
$$

this can be embedded in $M_{2^{k}}(\mathbb{C})$ if $n=2 k$ or $2 k+1$, as discussed in more detail in Appendix C.

As we shall see in the next section, each EJA has an associated probabilistic model. In the case of the EJAs $M_{n}(\mathbb{C})_{s a}, M_{n}(\mathbb{R})_{s a}$, and $M_{n}(\mathbb{H})_{s a}$, the associated state spaces $\Omega$ are the positive semidefinite trace- 1 self-adjoint matrices, which can be viewed as the density matrices associated with $n$-dimensional complex, real, or quaternionic Hilbert spaces respectively. In each case, the maximal number of mutually perfectly distinguishable states (a quantity we call the information capacity of a state space) is $n$. The 
state space of $V_{n}$ is an $n$-dimensional Euclidean ball, which has information capacity 2 , and so constitutes a "generalized bit". The associative EJA of dimension $n$ has a simplex with $n$ vertices as its state space, so it can be viewed as a classical system of information capacity $n$.

Classification If $A$ and $B$ are EJAs, their vector-space direct sum $A \oplus B$ is also an EJA under the obvious inner product and slot-wise Jordan product. In this case, $A$ and $B$ can be regarded as subalgebras of $A \oplus B$. More than that, they are Jordan ideals, that is, if $a \in A$ and $x \in A \oplus B$, then $a \bullet x \in A$ as well, and similarly for $B$. Conversely, if $A$ is any EJA and $A_{0} \leq A$ is a Jordan ideal, then so is $A_{1}:=A_{0}^{\perp}:=\left\{b \in A \mid \forall a \in A_{0} a \bullet b=0\right\}$, and $A \simeq A_{0} \oplus A_{1}$. Since $A$ is finite-dimensional, one can repeat this process so as to decompose $A$ into a direct sum $A=\bigoplus_{i} A_{i}$ of finitely many irreducible or simple EJAs $A_{i}$. (See Appendix A for further details.)

The EJAs $M_{n}(\mathbb{D})_{s a}$ for $\mathbb{D}=\mathbb{R}, \mathbb{C}, \mathbb{H}$, and the spin factors $V_{n}$, discussed above, are all simple. ${ }^{9}$ The Jordan-von Neuman-Wigner Classification Theorem [43] provides a near converse: every simple EJA is isomorphic to one of these types, i.e. isomorphic to a spin factor $V_{n}$, or to the self-adjoint part of a matrix algebra $M_{n}(\mathbb{D})$ where $\mathbb{D}$ is one of the classical division algebras $\mathbb{R}, \mathbb{C}$ or $\mathbb{H}$, or, if $n=3$, the octonions, $\mathbb{O}$. This last example, which is not embeddable into a complex matrix algebra, is called the exceptional Jordan algebra, or the Albert algebra.

A Jordan algebra that is embeddable in the self-adjoint part of a complex matrix algebra is said to be special. In addition to $M_{n}(\mathbb{C})_{s a}$, the simple EJAs $M_{n}(\mathbb{R})_{s a}, M_{n}(\mathbb{H})_{s a}$ and $V_{n}$ are all special. It follows from the classification theorem that any EJA decomposes as a direct sum $A_{s p} \oplus A_{e x}$ where $A_{s p}$ is special and $A_{e x}$ is a direct sum of copies of the exceptional Jordan algebra.

Operator commutation For each $a \in A$, define $L_{a}: A \rightarrow A$ to be the operation of Jordan multiplication by $a: L_{a}(x)=a \bullet x$ for all $x \in A$. Elements $a, b \in A$ are said to operator commute iff $L_{a} \circ L_{b}=L_{b} \circ L_{a}$. If $A$ is a Jordan subalgebra of $M_{s a}$, where $M$ is a complex $*$-algebra, then for all $x \in A$,

$$
4 L_{a}\left(L_{b} x\right)=a(b x+x b)+(b x+x b) a=a b x+a x b+b x a+x b a
$$

and similarly

$$
4 L_{b}\left(L_{a} x\right)=b a x+b x a+a x b+x a b .
$$

If $a$ and $b$ operator commute, the left-hand sides are equal. Subtracting, we have

$$
a b x+x b a-b a x-x a b=0
$$

or

$$
[a, b] x+x[b, a]=0
$$

\footnotetext{
${ }^{9}$ The $n$-dimensional associative EJA is not simple, rather it is a direct sum of $n$ copies of the one-dimensional EJA.
} 
which is to say, $[a, b] x-x[a, b]=0$. If $M$ is unital and $A$ is a unital subalgebra, so that $u_{A}=1_{\mathbf{M}}$, then setting $x=1_{M} \in A$ gives us $[a, b]=-[a, b]$, i.e., $a$ and $b$ commute in $M$.

Projections and the Spectral Theorem A projection in an EJA $A$ is an element $a \in A$ with $a^{2}=a$. If $p, q$ are projections with $p \bullet q=0$, we say that $p$ and $q$ are orthogonal. This implies that $\langle p, q\rangle=\langle p, p \bullet q\rangle=0 .{ }^{10}$ In this case, $p+q$ is another projection. A projection not representable as a sum of other projections is said to be minimal or primitive. A Jordan frame is a set $E \subseteq A$ of pairwise orthogonal minimal projections that sum to the Jordan unit. The Spectral Theorem for EJAs (see e.g. [26], Theorem III.1.1, or [4], Theorem 2.20 for an infinite-dimensional version) asserts that every element $a \in A$ can be expanded as a linear combination $a=\sum_{x \in E} t_{x} x$ where $E$ is some Jordan frame and $t_{x}$ is a coefficient in $\mathbb{R}$ for each $x \in E$. If $a$ has spectral decomposition $a=\sum_{x \in E} t_{x} x$, where $E$ is a Jordan frame, then $a \geq 0$ iff $t_{x} \geq 0$ for all $x \in E$. (The "if" direction is trivial; for the converse, notice that since $x, a \in A_{+}$, $\langle x, a\rangle=t_{x}\|x\|^{2} \geq 0$, whence, $t_{x} \geq 0$.

By summing over those $x \in E$ for which $t_{x}$ has a given value, we obtain a decomposition $\sum_{i} t_{i} p_{i}$ where $p_{i}$ are pairwise Jordan-orthogonal projections and the coefficients $t_{i}$ are distinct. In this form, the spectral decomposition is unique [26]. This gives us a functional calculus, as we can now define $f(a)=\sum_{i} f\left(t_{i}\right) p_{i}$ for any real-valued function $f$ defined on the set of coefficients $t_{i}$. In particular, every $a \in A_{+}$has a square root $\sqrt{a}=\sum_{i} \lambda_{i}^{1 / 2} p_{i}$ also in $A_{+}$.

Decomposing $A$ as an orthogonal direct sum $A=\bigoplus_{i} A_{i}$ of simple Jordan ideals $A_{i}$, the Jordan unit $u_{A}$ is the sum of the Jordan units $u_{A_{i}}$ of these ideals. It follows that each Jordan frame $E$ of $A$ is the disjoint union of Jordan frames $E_{i}$ belonging to the various simple ideals $A_{i}$. If $A$ is simple, the group of Jordan automorphisms acts transitively on the set of Jordan frames ([26], Theorem IV.2.5). It follows that all Jordan frames for a given euclidean Jordan algebra $A$ have the same number of elements. This number is called the rank of $A$. By the Classification Theorem, all simple Jordan algebras having rank 4 or higher are special.

\subsection{Euclidean Jordan algebras as probabilistic models}

As remarked earlier, any euclidean Jordan algebra $A$ can be regarded as an ordered real vector space, with positive cone $A_{+}=\left\{a^{2} \mid a \in A\right\}$. (That this is a cone is a non-trivial fact (see [26], Theorem III.2.1, or [3], pp. 36-28).) By the spectral theorem, $a=b^{2}$ for some $b \in A$ iff $a$ has a spectral decomposition $a=\sum_{i} \lambda_{i} x_{i}$ in which all the coefficients $\lambda_{i}$ are non-negative. It can also be shown (see [26], Proposition I.1.4) that $a$ belongs to the interior of $A_{+}$iff $\langle a, b\rangle>0$ for all nonzero $b \in A_{+}$. Using this, it follows that $a$ belongs to the interior of $A_{+}$iff it has a spectral decomposition $a=\sum_{x \in E} t_{x} x$ with all coefficients $t_{x}$ strictly positive. Hence, if $a$ belongs to the interior of $A_{+}$, so does $\sqrt{a}$.

The Jordan unit $u$ is also an order unit. Thus, any EJA can serve as a probabilistic

${ }^{10}$ In fact the converse is also true, cf. Ch. II Exercise 3 or Ch. III Exercise 7 in [26]. 
model, as defined in Section 2: physical states correspond to states qua normalized positive linear functionals on $A$, while measurement outcomes are represented by effects, i.e, elements $a \in A_{+}$with $0 \leq a \leq u$, and (discrete) observables, by sets $\left\{e_{i}\right\}$ of events with $\sum_{i} e_{i}=u$. Note that if $A=\bigoplus_{i=1}^{n} A_{i}$ where each $A_{i}$ is a copy of the one-dimensional Jordan algebra $\mathbb{R}$, then $A \simeq \mathbb{R}^{n}$, regarded as a commutative algebra (in particular, $a \bullet b=a b$ ). As a probabilistic system, this is classical, in the sense that it is simply the space of random variables on a finite sample space. From now on, when we speak of a classical system, this is what we have in mind.

As discussed earlier, the inner product on $A$ allows us to represent states internally, i.e., for every state $\alpha$ there exists a unique $a \in A_{+}$with $\alpha(x)=\langle a \mid x\rangle$ for all $x \in A$; conversely, every vector $a \in A_{+}$with $\langle a \mid u\rangle=1$ defines a state in this way. Now, if $a$ is a projection, i.e., $a^{2}=a$, let $\widehat{a}=\|a\|^{-2} a$ : then

$$
\left\langle\widehat{a} \mid u_{A}\right\rangle=\frac{1}{\|a\|^{2}}\left\langle a \mid u_{A}\right\rangle=\frac{1}{\|a\|^{2}}\left\langle a^{2} \mid u_{A}\right\rangle=\frac{1}{\|a\|^{2}}\langle a \mid a\rangle=1 .
$$

Thus, $\widehat{a}$ represents a state. A similar computation shows that $\langle\widehat{a} \mid a\rangle=1$. Thus, every projection, regarded as an effect, has probability 1 in some state.

Lemma 3.1. Let $A$ be an EJA, and let a be an effect, i.e. $a \in\left[0, u_{A}\right] \subseteq A_{+}$. Then a is a projection iff $\left\langle u_{A}, a\right\rangle=\langle a, a\rangle$.

Proof: If $a$ is a projection, then $\langle a, a\rangle=\left\langle u_{A} \bullet a, a\right\rangle=\left\langle u_{A}, a \bullet a\right\rangle=\left\langle u_{A}, a\right\rangle$. Conversely, suppose $\left\langle u_{A}, a\right\rangle=\langle a, a\rangle$. Let $a$ have spectral decomposition $a=\sum_{x \in E} t_{x} x$ where $E$ is a Jordan frame. We then have $\left\langle u_{A}, a\right\rangle=\sum_{x \in E} t_{x}\|x\|^{2}$, while $\langle a, a\rangle=\sum_{x \in E} t_{x}^{2}\|x\|^{2}$. Since $a$ is an effect, $0 \leq t_{x} \leq 1$ for every $x \in E$, so that $t_{x}^{2}\|x\|^{2}<t_{x}\|x\|^{2}$ unless $t_{x}=0$ or $t_{x}=1$. In order for the two sums above to be equal, therefore, we must have that $t_{x}=0$ or $t_{x}=1$ for every $x$. Setting $B=\left\{x \in E \mid t_{x}>0\right\}$, we have $a=\sum_{x \in B} x$, a projection.

When $A$ is special, i.e., a Jordan subalgebra of a matrix algebra, its order structure is inherited from that of the latter.

Proposition 3.2. Let $A \leq \mathbf{M}_{\text {sa }}$, i.e., $A$ is a Jordan subalgebra of a finite-dimensional complex matrix algebra $\mathbf{M}$. Then $A_{+}=A \cap \mathbf{M}_{+}$.

Proof: $A_{+} \subseteq \mathbf{M}_{+}$because squares in $A$ are squares in $\mathbf{M}_{+}$. For the converse, let $a \in A \cap \mathbf{M}_{+}$. By the spectral theorem for EJAs, we can express $a$ as a sum $a=\sum_{i} \lambda_{i} e_{i}$ where the $e_{i}$ are pairwise Jordan-orthogonal idempotents, i.e, $e_{i} \bullet e_{j}=0$ for $i \neq j$. Jordan-orthogonal idempotents in $A$ are again Jordan-orthogonal idempotents in $\mathbf{M}_{s a}$. Since Jordan-orthogonal idempotents in $\mathbf{M}_{s a}$ are orthogonal in the usual sense and $a \in \mathbf{M}_{+}$, it follows that the coefficients $\lambda_{i}$ are all non-negative, whence, $a \in A_{+}$.

Order-automorphisms The order structure of an EJA $A$, together with its inner product and order unit, entirely determines its Jordan structure, as a consequence of the Koecher-Vinberg theorem [45, 62] (discussed in more detail below). One manifestation 
of this is that a symmetry of $A$ - that is, an order-automorphism preserving the unit $u_{A}$ - is the same thing as a Jordan automorphism ([4], Theorem 2.80).

Another class of order automorphisms is given by the quadratic representations of certain elements of $A$. The quadratic representation of $a \in A$ is the mapping $U_{a}: A \rightarrow A$ given by

$$
U_{a}=2 L_{a}^{2}-L_{a^{2}}
$$

i.e., $U_{a}(x)=2 a \bullet(a \bullet x)-(a \bullet a) \bullet x$. These mappings have direct physical interpretations as filters in the sense of [66]. The following non-trivial facts will be used repeatedly in what follows:

Proposition 3.3. Let $a \in A$. Then

(a) $U_{a}$ is a positive mapping;

(b) If a lies in the interior of $A_{+}, U_{a}$ is invertible, with inverse given by $U_{a^{-1}}$;

(c) $e^{L_{a}}=U_{e^{a / 2}}$

Proof: For (a), see Theorem 1.25 of [4]; for (b), [4] Lemma 1.23 or [26], Proposition II.3.1. Part (c) is Proposition II.3.4 in [26].

Combining (a) and (b), $U_{a}$ is an order automorphism for every $a$ in the interior of $A_{+}$. Regarding (c), note that $e^{L_{a}}$ is the ordinary operator exponential. Therefore if $a$ is in the interior of $A_{+}$, then $\phi_{t}:=e^{t L_{a}}=U_{\frac{t}{2} a}$ is a one-parameter group of order-automorphisms with $\phi^{\prime}(0)=L_{a}$. Therefore, for all $a$ in the interior of $A_{+}, U_{a}$ is in the connected component $\operatorname{Aut}_{0}(A)$ of the identity in the group $\operatorname{Aut}(A)$ of order-automorphisms of $A$.

Notice that $U_{a}\left(u_{A}\right)=2 a^{2}-a^{2}=a^{2}$ If $a$ belongs to the interior of $A_{+}$, then so does $\sqrt{a}$; thus, by the remarks above, $U_{\sqrt{a}} \in \operatorname{Aut}_{0}(A)$ and $U_{\sqrt{a}}\left(u_{A}\right)=a$. It follows that $\operatorname{Aut}_{0}(A)$, and hence also the full group $\operatorname{Aut}(A)$ of order-automorphisms of $A$, act transitively on the interior of $A_{+}$: if $a, b$ belong to the interior of $A_{+}$, then $U_{\sqrt{b}} \circ U_{\sqrt{a}}^{-1}$ maps $a$ to $b$. In other words, $A_{+}$is homogeneous.

EJAs as dynamical models Henceforth, we will write $G(A)$ for the identity component $\operatorname{Aut}_{0}(A)$ of an EJA $A$. As this notation suggests, we henceforth regard an EJA $A$ as a dynamical probabilistic model with $G(A)$ as its dynamical group. This is a reasonable choice. First, elements of $G(A)$ are exactly those automorphisms of $A_{+}$that figure in the system's possible dynamics, as elements of one-parameter groups of automorphisms. This suggests that the "physical" dynamical group of a dynamical model based on $A$ should at least be a subgroup of $G(A)$, so that the latter is the least constrained choice. Moreover, $G(A)$, like the full group of order-automorphisms, acts transitively on the interior of the cone $A_{+}$, and its unit-preserving subgroup acts transitively on the set of Jordan frames, i.e., maximally informative sharp observables or, equivalently, on maximal lists $\left(\alpha_{1}, \ldots, \alpha_{n}\right)$ of sharply-distinguishable states. These transitivity properties, abstracted from the Jordan-algebraic setting, were among the postulates used (in somewhat different ways) in [63, 64] and [15] to derive the Jordan 
structure of probabilistic models, so it is not unreasonable to require that the dynamical group enjoy them.

If $\phi$ is any order-automorphism with $\phi\left(u_{A}\right)=a^{2} \in A_{+}$, then $U_{a}^{-1} \circ \phi$ is a symmetry of $A$. Hence, every order-automorphism of $A$ decomposes as $\phi=U_{a} \circ g$ where $g$ is a symmetry. As we observed earlier, $a$ can be chosen to belong to the interior of $A_{+}$; it can be shown that with this choice, the decomposition is unique ([26], III.5.1)

The Koecher-Vinberg Theorem As we have seen, the positive cone $A_{+}$of an EJA is homogeneous, and also self-dual with respect to $A$ 's inner product. Conversely, let $A$ be an homogenous finite-dimensional ordered vector space with a self-dualizing inner product. Choosing any order-unit $u$ invariant under positive orthogonal transformations, there exists a unique bilinear product on $A$ making $A$, with the given inner product, a euclidean Jordan algebra, and the chosen element $u$, the Jordan unit. This is the content of the Koecher-Vinberg Theorem ([45, 62]; see also [26, 56]). While we make no use of this result here, it is at the center of efforts to provide an operational motivation for euclidean Jordan algebras as models of physical systems, e.g., in [66, 15].

\subsection{Representations of EJAs}

A representation of a Jordan algebra $A$ is a Jordan homomorphism $\pi: A \rightarrow M_{n}(\mathbb{C})_{s a}$ for some $n .^{11}$ So a Jordan algebra is special iff it has an injective, or faithful, representation. Recall that every EJA decomposes as a direct sum $A=A_{s p} \oplus A_{e x}$, where $A_{s p}$ is special and $A_{e x}$ has no nontrivial representations. The latter, in turn, is a direct sum of copies of the exceptional EJA $M_{3}(\mathbb{O})_{s a}$. See [4], Theorem 4.3 for details.

Standard Representations For the non-exceptional simple EJAs, it will be useful to record what we will call their standard representations. It will also prove helpful to adopt the following abbreviations:

$$
R_{n}=M_{n}(\mathbb{R})_{s a} ; C_{n}=M_{n}(\mathbb{C})_{s a}, Q_{n}=M_{n}(\mathbb{H})_{s a}
$$

As above, we write $V_{n}$ for the spin factor $\mathbb{R} \times \mathbb{R}^{n}$. With this notation we have obvious embeddings $R_{n} \leq C_{n}=M_{n}(\mathbb{C})_{s a}$. For $Q_{n}$, note that a quaternion $a+b i+c j+d k$ can be written as $(a+b i)+(c+d i) j$, and so, can be represented by the pair of complex numbers $(a+b i, c+d i)$. Thus, any $n \times n$ matrix of quaternions can be represented as a $2 n \times 2 n$ complex matrix having the form

$$
\left(\begin{array}{cc}
\Gamma_{1} & \Gamma_{2} \\
-\bar{\Gamma}_{2} & \bar{\Gamma}_{1}
\end{array}\right)
$$

where the blocks $\Gamma_{1}$ and $\Gamma_{2}$ are $n \times n$ complex matrices. This gives us a faithful representation of $Q_{n}$ in $M_{2 n}(\mathbb{C})$, known as the symplectic representation [28]. There

\footnotetext{
${ }^{11}$ This is a finite dimensional, concrete representation. For the finite-dimensional algebras we are concerned with, this definition suffices.
}

Accepted in 〈 \uantum 2020-10-07, click title to verify. Published under CC-BY 4.0. 
is also what we will call a standard representation of $V_{n}$ in $M_{2^{k}}(\mathbb{C})$, where $n=2 k$ or $2 k+1$. This is less obvious; the details are given in Appendix C.

Involutions and Reversibility By an involution on a complex *-algebra $M$, we mean a *-anti-automorphism of $M$ of period 2 - in more detail, a linear mapping $\Phi: M \rightarrow M$ such that $\Phi\left(a^{*}\right)=\Phi(a)^{*}, \Phi(a b)=\Phi(b) \Phi(a)$, and $\Phi(\Phi(a))=a$ for all $a \in M$. A real involution is defined similarly, except that the mapping is only required to be reallinear. ${ }^{12}$ It is straightforward that the set $M_{s a}^{\Phi}=\left\{a \in M \mid a=a^{*}=\Phi(a)\right\}$ of all selfadjoint fixed-points of $M$ under $\Phi$ is a Jordan subalgebra of $M_{s a}$. Indeed, if $a, b \in M_{s a}^{\phi}$, then $a \cdot b=\frac{1}{2}(a b+b a) \in M_{s a}$, and

$$
\Phi(a b+b a)=\Phi(b) \Phi(a)+\Phi(a) \Phi(b)=b a+a b=a b+b a
$$

so that $a \bullet b \in M_{s a}^{\Phi}$ as well. In fact, more is true: if $a_{1}, \ldots, a_{n} \in M_{s a}^{\Phi}$, then

$$
\Phi\left(a_{1} \cdots a_{n}+a_{n} \cdots a_{1}\right)=a_{n} \cdots a_{1}+a_{1} \cdots a_{n}
$$

so that $a_{1} \cdots a_{n}+a_{n} \cdots a_{1} \in M_{s a}^{\Phi}$.

Definition 3.4. A Jordan subalgebra $A$ of $M_{s a}$ is said to be reversible if $a_{1}, \ldots, a_{n} \in$ $A \Longrightarrow a_{1} \cdots a_{n}+a_{n} \cdots a_{1} \in A$. An abstract EJA $A$ is reversible iff it has a faithful (that is, injective) representation as a reversible Jordan subalgebra of some $*$-algebra $M$. If all of $A$ 's faithful representations are reversible, then $A$ is said to be universally reversible (hereafter: UR). ${ }^{13}$

In this language, then, all Jordan algebras of the form $M_{s a}^{\Phi}$ are reversible. All selfadjoint parts of real and complex matrix algebras are universally reversible, as are the quaternionic ones of rank 3 and higher (whence, all EJAs of rank $\geq 4$ are UR). The quaternionic bit $M_{2}(\mathbb{H})_{s a}$, which is isomorphic to $V_{5}$, is not universally reversible, but it is reversible, since its standard embedding into $M_{4}(\mathbb{C})$ is reversible: indeed, it's the set fixed points of the involution $\Phi(x)=\left(\sigma_{y} \otimes \mathbf{1}_{2}\right) x^{T}\left(\sigma_{y} \otimes \mathbf{1}_{2}\right)$ where $\sigma_{y}=-\overline{\sigma_{y}}$ is the usual Pauli $y$-matrix. Spin factors $V_{n}$ with $n=4$ or $n \geq 6$ are not reversible at all. For details, see [30]. Thus, the reversible simple EJAs are just those of the forms $M_{n}(\mathbb{R})_{s a}$, $M_{n}(\mathbb{C})_{s a}$ and $M_{n}(\mathbb{H})_{s a}$.

The universal representation In addition to the standard representations discussed above, every special EJA has a universal representation.

Definition 3.5. A universal $C^{*}$ algebra for a euclidean Jordan algebra $A$ is a complex *-algebra $C^{*}(A)$, plus a Jordan homomorphism $\psi_{A}: A \rightarrow C^{*}(A)_{s a}$, such that for any $C^{*}$-algebra $\mathbf{M}$ and any Jordan homomorphism $\phi: A \rightarrow \mathbf{M}_{s a}$, there exists a unique *-homomorphism $\widehat{\phi}: C^{*}(A) \rightarrow \mathbf{M}$ with $\phi=\widehat{\phi} \circ \psi_{A}$.

\footnotetext{
${ }^{12}$ Our usage is slightly nonstandard here: an involution on a complex $*$-algebra is more frequently defined to be a conjugate-linear. The only involution in this sense that will concern us is $a \mapsto a^{*}$.

${ }^{13}$ Some authors, for example Hanche-Olsen in [30], define universal reversibility as reversibility in all representations, not just all faithful representations. The two definitions are equivalent, as will become apparent below.
} 
Note that the uniqueneness of the $*$-homomorphism $\widehat{\phi}: C^{*}(A) \rightarrow \mathbf{M}$ is equivalent to $C^{*}(A)$ being generated by $\psi_{A}(A)$ as a $C^{*}$ algebra, which is how the definition is more usually presented. For the existence of $C^{*}(A)$, see [4] or [31]. Universal $C^{*}$-algebras for a given EJA $A$ are unique up to canonical $*$-isomorphism, warranting our speaking of "the" universal $C^{*}$-algebra of $A .{ }^{14}$ It is easy to see that any Jordan homorphism $A \rightarrow B$ lifts, via $\psi_{A}$ and $\psi_{B}$, to a $C^{*}$-homomorphism $C^{*}(A) \rightarrow C^{*}(B)$, and that this lifting respects composition of Jordan homomorphisms, so that $C^{*}(\cdot)$ defines a functor from the category of EJAs to the category of complex $*$-algebras. It can be shown that this functor is exact ([30], Theorem 4.1). It is an important fact that $A$ is exceptional iff $C^{*}(A)=\{0\}$. Otherwise, the representation $\psi_{A}$ of $A$ in $C^{*}(A)$ is faithful, and takes the unit of $A$ to the unit of $C^{*}(A)$. In this case, we will often identify $A$ with its image $\psi_{A}(A)$ in $C^{*}(A)$, referring to this (and to the homomorphism $\psi_{A}$ ) as the universal embedding of $A$.

We can now see that reversibility in all faithful representations implies reversibility in all representations. It is straightforward that any representation factoring through a reversible representation is also reversible. If $A$ is universally reversible in the sense of Definition 3.4, then the universal representation, which is faithful, is also reversible. Since every representation factors through this one, every representation of $A$ is reversible.

The canonical involution If $A$ is special, $C^{*}(A)$ comes equipped with a unique involution that fixes every point of $A$. To see this, note that that the opposite algebra $C^{*}(A)^{\text {op }}$ (the same vector space, equipped with reversed multiplication) is equally a universal $C^{*}$-algebra for $A$. Hence, there is a unique $*$-isomorphism $\Phi_{A}: C^{*}(A)^{\text {op }} \rightarrow C^{*}(A)$ fixing all points of $A$. We can equally well regard $\Phi$ as a $*$-antiautomorphism of $C^{*}(A)$; so regarded, $\Phi$ is self-inverse, hence an involution.

Definition 3.6. We call the involution $\Phi: C^{*}(A) \rightarrow C^{*}(A)$ just described, the canonical involution, $\Phi_{A}$, on $C^{*}(A)$.

As discussed above, the self-adjoint fixed-points of an involution $\Phi$ on a complex *-algebra $M$ constitute a Jordan subalgebra, $M_{s a}^{\Phi}$, of $M_{s a}$. With $\Phi_{A}$ the canonical involution on $C^{*}(A)_{s a}$, we have a Jordan embedding $A \leq C^{*}(A)_{s a}^{\Phi}$.

Proposition 3.7 ([30], Lemma 4.2 and Theorem 4.4). With notation as above, $A$ is UR iff $A=C^{*}(A)_{s a}^{\Phi}$. More generally, if $A$ is $U R$ and there exist an embedding $A \leq M_{s a}$ for a complex *-algebra $M$, and an involution $\Phi$ on $M$ fixing points of $A$, then the *-subalgebra of $M$ generated by $A$ is isomorphic to $C^{*}(A)$ and $\Phi$, restricted to this subalgebra, is the canonical involution.

This characterization allows the explicit computation of universal $C^{*}$-algebras of UR EJAs [30]. For instance, $R_{n}=M_{n}(\mathbb{R})_{s a}$ generates $M_{n}(\mathbb{C})_{s a}$ as a (complex) *-algebra,

\footnotetext{
${ }^{14}$ In fact, by privileging any particular construction of $C^{*}(A)$, we can take this literally.
} 
in which it sits as the set of symmetric self-adjoint matrices: in other words, the set of fixed points of the involution $\Phi(a)=a^{T}$. Thus, $C^{*}\left(R_{n}\right)=M_{n}(\mathbb{C})$. Similarly, the image of $M_{n}(\mathbb{H})_{s a}$ under the standard (symplectic) embedding into $M_{2 n}(\mathbb{C})=M_{2}\left(M_{n}(\mathbb{C})\right)$ is fixed by the involution $\Phi(a)=-J a^{T} J$ where $J$ is the block matrix

$$
J=\left(\begin{array}{rr}
0 & \mathbf{1} \\
-1 & 0
\end{array}\right)
$$

with 1 the $n \times n$ identity matrix. For a third example, consider the embedding $M_{n}(\mathbb{C})_{s a} \rightarrow\left(M_{n}(\mathbb{C}) \oplus M_{n}(\mathbb{C})\right)_{s a}$ given by $\psi(a)=\left(a, a^{T}\right)$ : the image of this embedding generates $M_{n}(\mathbb{C}) \oplus M_{n}(\mathbb{C})$ as a $*$-algebra, and is exactly the set of fixed-points of the involution $\Phi(a, b)=\left(b^{T}, a^{T}\right)$. Thus, $C^{*}\left(C_{n}\right)=M_{n}(\mathbb{C}) \oplus M_{n}(\mathbb{C})$. Note, in passing, that this also shows that there is no involution on $M_{n}(\mathbb{C})$ fixing points of $C_{n}=M_{n}(\mathbb{C})_{s a}$. (Otherwise, $M_{n}(\mathbb{C})$ would be the universal $*$-algebra for $C_{n}$. )

The universal $C^{*}$-algebras for all simple, non-exceptional EJAs are are summarized in Table 1 below, along with the canonical involutions in the UR cases. For a spin factor $V_{n}$, the universal $C^{*}$-algebra is the complex Clifford algebra $\operatorname{Cliff}_{\mathbb{C}}(n)$ on $n$ generators [30]; these are tabulated separately in Table 1(b).

For contrast, Table 1(c) lists the complex matrix algebras into which $R_{n}, C_{n}$ and $Q_{n}$ are standardly embedded. We also list in Fig. 1 (c) algebras supporting what we are calling standard embeddings of the spin factors $V_{n}$; these agree with the universal ones for $n=2 k$, but for $n=2 k+1$, embed $V_{n}$ in $M_{2^{k}}(\mathbb{C})$ rather than the $M_{2^{k}}(\mathbb{C}) \oplus M_{2^{k}}(\mathbb{C})$ of the universal embedding. Thus, all the targets of embeddings in Fig. 1(c) are simple complex matrix algebras.

\begin{tabular}{l|l|l}
$A$ & $C^{*}(A)$ & $\Phi$ \\
\hline$R_{n}$ & $M_{n}(\mathbb{C})$ & $a \mapsto a^{T}$ \\
$C_{n}$ & $M_{n}(\mathbb{C}) \oplus M_{n}(\mathbb{C})$ & $(a, b) \mapsto\left(b^{T}, a^{T}\right)$ \\
$Q_{n}$ & $M_{2 n}(\mathbb{C})$ if $n>2$ & $a \mapsto-J a^{T} J$ \\
$V_{n}$ & $\operatorname{Cliff}_{\mathbb{C}}(n)$ &
\end{tabular}

(a)

Universal embeddings

\begin{tabular}{l|l}
$n\left(k \in \mathbb{N}_{+}\right)$ & $\operatorname{Cliff}_{\mathbb{C}}(n)$ \\
\hline $2 k$ & $M_{2^{k}}(\mathbb{C})$ \\
$2 k+1$ & $M_{2^{k}}(\mathbb{C}) \oplus M_{2^{k}}(\mathbb{C})$
\end{tabular}

(b)

Clifford algebras

\begin{tabular}{l|l}
$A$ & $\mathbf{M}$ \\
\hline$R_{n}$ & $M_{n}(\mathbb{C})$ \\
$C_{n}$ & $M_{n}(\mathbb{C})$ \\
$Q_{n}$ & $M_{2 n}(\mathbb{C})$ \\
$V_{2 k}$ & $M_{2^{k}}(\mathbb{C})$ \\
$V_{2 k+1}$ & $M_{2^{k}}(\mathbb{C})$
\end{tabular}

(c)

Standard embeddings

Table 1: Universal and standard embeddings 
Note that the spin factors $V_{2}, V_{3}, V_{5}$ correspond to the three types of quantum bits: $V_{2} \simeq R_{2} ; V_{2} \simeq C_{2}$ and $V_{5} \simeq Q_{2}$. This last, together with line 2 of Fig. 1 table (b), gives us the missing item in table (a):

$$
C^{*}\left(Q_{2}\right) \simeq M_{4}(\mathbb{C}) \oplus M_{4}(\mathbb{C}) .
$$

It will be helpful to record here two further facts about universal $C^{*}$ algebras. First, $C^{*}(A \oplus B)=C^{*}(A) \oplus C^{*}(B)$. This follows from the exactness of the $C^{*}()$-functor. Combining this with Proposition 3.7, it follows that if $A$ and $B$ are both UR, so is $A \oplus B$. Details can be found in Appendix A.

\subsection{The universal tensor product}

The universal representation allows one to define a natural tensor product of EJAs, first studied by H. Hanche-Olsen [30]:

Definition 3.8. The universal tensor product of two EJAs $A$ and $B$, denoted $A \widetilde{\otimes} B$, is the Jordan subalgebra of $C^{*}(A) \otimes C^{*}(B)$ (the tensor product of $C^{*}(A)$ and $C^{*}(B)$ as finite-dimensional $*$-algebras) generated by $\psi_{A}(A) \otimes \psi_{B}(B)$.

Since $C^{*}(\mathbb{R})=\mathbb{C}$, trivially we have $\mathbb{R} \widetilde{\otimes} A \simeq A$. Other examples are discussed below. Some important general facts about the universal tensor product are collected in the following:

Proposition 3.9. Let $A, B$ and $C$ denote EJAs.

(a) If $\phi: A \rightarrow C, \psi: B \rightarrow C$ are unital Jordan homomorphisms with operatorcommuting ranges, then there exists a unique Jordan homomorphism $A \widetilde{\otimes} B \rightarrow C$ taking $a \otimes b$ to $\phi(a) \bullet \psi(b)$ for all $a \in A, b \in B$.

(b) $C^{*}(A \widetilde{\otimes} B)=C^{*}(A) \otimes C^{*}(B)$ and $\Phi_{A \widetilde{\otimes} B}=\Phi_{A} \otimes \Phi_{B}$.

(c) $A \widetilde{\otimes} B$ is universally reversible unless one of the factors has a one-dimensional summand and the other has a representation onto a spin factor $V_{n}$ with $n=4$ or $n \geq 6$.

(d) If $A$ is universally reversible, then $A \widetilde{\otimes} M_{n}(\mathbb{C})_{s a}=\left(C^{*}(A) \otimes M_{n}(\mathbb{C})\right)_{s a}$.

(e) $u_{A \widetilde{\otimes} B}=u_{A} \otimes u_{B}=u_{C^{*}(A \widetilde{\otimes} B)}$.

Proof: (a), (c), and (d) are Propositions 5.2, 5.3 and 5.4, respectively, in [30]; (b) is observed in the vicinity of these propositions in [30], while (e) follows easily from the fact that $\psi^{A}\left(u_{A}\right)=u_{C^{*}(A)}$.

Table 1 (c) shows that if $A$ and $B$ are simple and nontrivial, $A \widetilde{\otimes} B$ will always be UR, 
and hence, by Proposition 3.7 and Proposition 3.9 (b), the fixed-point set of $\Phi_{A} \otimes \Phi_{B}$. Using this, one can compute $A \widetilde{\otimes} B$ for simple $A$ and $B$ (with $n, k>2$ in the case of $Q_{n}$ and $Q_{k}$ ) [30]:

\begin{tabular}{c|ccc}
$\widetilde{\otimes}$ & $R_{k}$ & $C_{k}$ & $Q_{k}$ \\
\hline$R_{n}$ & $R_{n k}$ & $C_{n k}$ & $Q_{n k}$ \\
$C_{n}$ & $C_{n k}$ & $C_{n k} \oplus C_{n k}$ & $C_{2 n k}$ \\
$Q_{n}$ & $Q_{n k}$ & $C_{2 n k}$ & $R_{4 n k}$
\end{tabular}

Table 2: Universal tensor products of simple UR EJAs

For $Q_{2} \widetilde{\otimes} Q_{2}$, we obtain the direct sum of four copies of $R_{16}=M_{16}(\mathbb{R})_{s a}$. The details can be found in [27].

In the next two sections, we are going to explore in some detail the possibilities for forming dynamical composites, in the sense of our Definition 2.1, of Jordan-algebraic systems. As will emerge, the universal tensor product $A \widetilde{\otimes} B$ is such a composite of $A$ and $B$, though not the only one. In this connection, notice that $A \widetilde{\otimes} B$ is in general a larger vector space than $A \otimes B$. In other words, as a composite of $A$ and $B, A \widetilde{\otimes} B$ is not necessarily locally tomographic. Indeed, this will typically be the case for composites of EJAs, as underlined by the following finite-dimensional case of a result of HancheOlsen (valid more generally for JC algebras), which was used in [17] to show that the only EJAs having locally tomographic composites with a qubit are the complex quantum systems (those whose Jordan algebras are the self-adjoint parts of complex matrix algebras):

Proposition 3.10 ([30], Theorem 5.5). Let $A$ be an EJA, and suppose that the vectorspace tensor product $A \otimes M_{2}(\mathbb{C})$ is a Jordan algebra with respect to a bilinear product - such that

(i) $\left(u_{A} \otimes b\right)^{2}=u_{A} \otimes b^{2}$ and $(a \otimes \mathbf{1})^{2}=a^{2} \otimes \mathbf{1}$,

(ii) $(a \otimes \mathbf{1}) \cdot\left(u_{A} \otimes b\right)=a \otimes b$,

(iii) $a \otimes \mathbf{1}$ and $u_{A} \otimes b$ operator commute for all $a \in A$ and $b \in M_{2}(\mathbb{C})$, where 1 is the identity in $M_{2}(\mathbb{C})$. Then $A$ is the self-adjoint part of a complex matrix algebra.

\section{Composites of Jordan-Algebraic Systems}

As discussed in section 3.2, we can regard an EJA $A$ as a dynamical probabilistic model with dynamical group $G(A)$. If $A$ and $B$ are EJAs, thus regarded as models of probabilistic physical systems, we would like to know what possibilities exist for forming a Jordan-algebraic dynamical composite $A B$. We shall actually impose a further, but we think natural, condition on such composites, namely condition (b) in the definition below: 
Definition 4.1. A composite of EJAs $A$ and $B$ is an EJA $A B$, plus a bilinear mapping $\pi: A \otimes B \rightarrow A B$, such that

(a) $\pi$ makes $(A B, G(A B))$ a dynamical composite of $(A, G(A))$ and $(B, G(B))$, in the sense of Definition 2.3,

(b) $(\phi \otimes \psi)^{\dagger}=\phi^{\dagger} \otimes \psi^{\dagger}$ for all $\phi, \psi \in G(A)$, and

(c) $A B$ is generated, as a Jordan algebra, by (images of) pure tensors.

By Lemma 2.2, the mapping $\pi: A \otimes B \rightarrow A B$ is injective; hence, we can, and shall, identify $A \otimes B$ with its image in $A B$, writing $\pi(a, b)$ as $a \otimes b$.

Condition (b) is rather strong, but natural if we keep in mind that our ultimate aim is to construct dagger-compact categories of EJAs. Regarding condition (c), suppose $\pi: A \times B \rightarrow A B$ satisfied only (a) and (b): letting $A \odot B$ denote the Jordan subalgebra of $A B$ generated by $\pi(A \otimes B)$, one can show (Appendix D) that the co-restriction of $\pi$ to $A \odot B$ also satisfies (a) and (b); thus, any composite in the weaker sense defined by (a) and (b) contains a composite satisfying all three conditions.

In Section 5, Proposition 5.4, we will show that $A \widetilde{\otimes} B$ is a composite in the sense of Definition 4.1. The main result of the present section is to show that any such composite $A B$ is a direct summand of $A \widetilde{\otimes} B$. In view of Table 2 , this severely limits the possibilities for $A B$.

\subsection{The identity $(\boldsymbol{a} \otimes \boldsymbol{u}) \bullet(\boldsymbol{x} \otimes \boldsymbol{y})=(\boldsymbol{a} \bullet \boldsymbol{x}) \otimes \boldsymbol{y}$.}

At this point, we have limited information about how the Jordan structure of a composite $A B$ interacts with the Jordan structures of $A$ and $B$. However, we shall now establish, for any $a \in A$ and any $x, y \in B$, the identity $(a \otimes u) \bullet(x \otimes y)=(a \bullet x) \otimes y-$ in other words, that $L_{a \otimes u_{B}}$ acts on $A \otimes B \leq A B$ as $L_{a} \otimes \mathbf{1}_{B}$, where $\mathbf{1}_{B}$ is the identity operator on $B$.

One-parameter groups and exponentials It will be helpful first to recall some basic facts about operator exponentials, or, equivalently, one-parameter groups of linear operators on finite-dimensional spaces (see, e.g., [22]). Let $V$ be a finite-dimensional real vector space, and $X$, a linear operator on $V$. Recall that $\phi(t):=e^{t X}$ is the unique function $\mathbb{R} \rightarrow \mathcal{L}(V)$ satisfying the initial-value problem

$$
\phi^{\prime}(t)=X \phi(t) ; \quad \phi(0)=\mathbf{1}
$$

(where 1 is the identity operator on $V$ ). In particular, $\phi^{\prime}(0)=X$. The function $\phi$ satisfies $\phi(t+s)=\phi(t) \phi(s)$ and hence, $\phi(t) \phi(-t)=\phi(0)=\mathbf{1}$, hence, as $\phi(0)=\mathbf{1}, \phi(t)$ is invertible, with $\phi(t)^{-1}=\phi(-t)$. In other words, $\phi$ is a one-parameter group of linear operators on $V$. Conversely, if $\phi: \mathbb{R} \rightarrow \mathcal{L}(V)$ is any continuous one-parameter group of 
linear operators on $V$, then $\phi$ is differentiable, and $\phi(t)=e^{t X}$ where $X=\phi^{\prime}(0)$. Notice, also, that in such a case we have

$$
X a=\left.\frac{d}{d t} \phi(t) a\right|_{t=0}
$$

for any vector $a \in V$.

For later reference, the following lemma collects some standard facts:

Lemma 4.2. Let $X, Y$ be linear operators on a finite-dimensional inner product space $V$. Then

(a) $X$ commutes with $e^{t X}$ for all $t$;

(b) If $e^{t X}$ commutes with $e^{s Y}$ for all $t, s$, then $X$ commutes with $Y$;

(c) $\left(e^{t X}\right)^{\dagger}=e^{t X^{\dagger}}$.

Note that, by (c), if $\phi(t)$ is a one-parameter group with $\phi^{\prime}(0)=X$ hermitian, then $\phi(t)$ is hermitian for all $t$, and conversely.

Now let $A$ be an EJA. For $a \in A$, define

$$
\phi_{a}(t):=e^{t L_{a}}=e^{L_{t a}}
$$

i.e., $\phi_{a}$ is the solution to the initial-value problem $\frac{d}{d t} \phi_{a}=L_{a} \phi_{a}, \quad \phi_{a}(0)=1$. By part (c) of Proposition 3.3, $\phi_{a}(t)=U_{e^{t a / 2}}$; by part (b) of the same Proposition, this last is a positive mapping. Since $e^{t L_{a}}$ is invertible with inverse $e^{-t L_{a}}=e^{L_{-t a}}, \phi_{a}(t)$ is an order-automorphism belonging to $G(A)$. It follows that $L_{a}$ belongs to $\mathfrak{g}_{A}$, the Lie group of the identity component $G(A)$ of $A$. Note that $\left\langle L_{a} x, y\right\rangle=\langle a x, y\rangle=\langle x, a y\rangle=\left\langle x, L_{a} y\right\rangle$ for all $x, y \in A$; that is, $L_{a}$ is self-adjoint. One can show that, conversely, a self-adjoint element of $\mathfrak{g}_{A}$ has the form $L_{a}$ for a unique $a \in A$. (See [26], pp. 6 and 49, for the details.)

We are now ready for the main result of this sub-section.

Proposition 4.3. Let $A B$ be a composite (in the sense of Definition 4.1) of Jordan algebras $A$ and $B$. Then the mapping $a \mapsto a \otimes u_{B}$ is a Jordan homomorphism from $A$ into $A B$. That is, for all $a, x \in A$ and $b, y \in B$,

$$
\left(a \otimes u_{B}\right) \bullet(x \otimes y)=(a \bullet x) \otimes y \quad \text { and }\left(u_{A} \otimes b\right) \bullet(x \otimes y)=x \otimes(b \bullet y)
$$

We shall refer to (1) as the fundamental identity.

Proof: We prove the first identity; the second is handled similarly. Let $\phi(t)$ be a oneparameter group of order automorphisms of $A$ with $\phi^{\prime}(0)=L_{a}$, that is, $\phi(t)=e^{t L_{a}}$. 
Then $\psi(t):=\phi(t) \otimes \mathbf{1}$ is a one-parameter group of automorphisms on $A B$, by condition (b) of Definition 2.3. Let $Y=\psi^{\prime}(0) \in \mathfrak{g}_{A B}$; then, for all $x \in A$ and $y \in B$,

$$
\begin{aligned}
Y(x \otimes y) & =\left[\frac{d}{d t} \psi(t)\right]_{t=0}(x \otimes y) \\
& =\left[\frac{d}{d t}(\psi(t)(x \otimes y))\right]_{t=0} \\
& =\left[\frac{d}{d t}(\phi(t) x \otimes y)\right]_{t=0} \\
& =\left(\left[\frac{d}{d t} \phi(t)\right]_{t=0} x\right) \otimes y=L_{a} x \otimes y=a x \otimes y .
\end{aligned}
$$

Using condition (b) of Definition 4.1, the hermiticity of $\phi^{\prime}(0)=L_{a}$, and the consequent hermiticity of $\phi(t)$ (see the discussion following Lemma 4.2), we have

$$
\left(\phi_{a}(t) \otimes \mathbf{1}\right)^{\dagger}=\phi_{a}(t)^{\dagger} \otimes \mathbf{1}=\phi_{a}(t) \otimes \mathbf{1}
$$

Hence, $Y$ is self-adjoint. As discussed above, it follows that there exists some $v \in A B$ with $Y=L_{v}$ on $A \otimes B$. Thus,

$$
v \bullet(x \otimes y)=L_{v}(x \otimes y)=Y(x \otimes y)=(a \bullet x) \otimes y .
$$

Setting $x=u_{A}$ and $y=u_{B}$, we have $v=v \bullet u_{A B}=v \bullet\left(u_{A} \otimes u_{B}\right)=\left(a \bullet u_{A}\right) \otimes u_{B}=a \otimes u_{B}$, which gives the advertised result.

One immediate consequence of the fundamental identity (1) is that, for any composite $A B$ of EJAs, $A \otimes u_{B}=\left\{a \otimes u_{B} \mid a \in A\right\}$ is a Jordan subalgebra of $A B$ isomorphic to $A$. In particular, since $A \otimes \mathbb{R}=A \otimes 1$, any composite of an EJA $A$ with the one-dimensional Jordan algebra $\mathbb{R}$ is canonically isomorphic to $A$.

The fundamental identity can be read as asserting that $L_{a} \otimes \mathrm{id}_{B}$ and $\mathrm{id}_{A} \otimes L_{b}$ act on $A \otimes B \leq A B$ in the expected way for all $a \in A, b \in B$. Recalling that, for $a \in A$, the mapping $U_{a}: A \rightarrow A$ is defined by $U_{a}=2 L_{a}^{2}-L_{a^{2}}$, we have the

Corollary 4.4. In any composite $A B$ of EJAs $A$ and $B$, and for any $a \in A, b \in B$, $U_{a \otimes u_{B}}$ and $U_{u_{A} \otimes b}$ act on $A \otimes B$ as $U_{a} \otimes i d_{B}$ and $i d_{A} \otimes U_{b}$, respectively.

Another, very important, consequence of Proposition 4.3 is the following:

Proposition 4.5. Let $p \in A$ and $q \in B$ be projections. Then $p \otimes q$ is a projection in $A B$, for any composite $A B$ of $A$ and $B$.

Proof: By Lemma 3.1, if $A$ is an EJA, an effect $a \in A_{+}$is a projection iff $\left\langle u_{A} \mid a\right\rangle=$ $\langle a \mid a\rangle$. Certainly, $p \otimes q$ is an effect in $A B_{+}$. Now note that, by repeated application of 
Proposition 4.3, plus the fact that $u_{A} \otimes u_{B}=u_{A B}$ and the associativity of the inner product, we have

$$
\begin{aligned}
\left\langle u_{A} \otimes u_{B}, p \otimes q\right\rangle & =\left\langle u_{A} \otimes u_{B}, p \bullet p \otimes q\right\rangle \\
& =\left\langle u_{A} \otimes u_{B},\left(p \otimes u_{B}\right) \bullet(p \otimes q)\right\rangle \\
& =\left\langle\left(u_{A} \otimes u_{B}\right) \bullet\left(p \otimes u_{B}\right), p \otimes q\right\rangle \\
& =\left\langle p \otimes u_{B}, p \otimes q\right\rangle \\
& =\left\langle p \otimes u_{B}, p \otimes q \bullet q\right\rangle \\
& =\left\langle p \otimes u_{B},\left(u_{A} \otimes q\right) \bullet(p \otimes q)\right\rangle \\
& =\left\langle\left(p \otimes u_{B}\right) \bullet\left(u_{A} \otimes q\right), p \otimes q\right\rangle \\
& =\langle p \otimes q, p \otimes q\rangle .
\end{aligned}
$$

It follows that $p \otimes q$ is a projection.

Corollary 4.6. Let $p, q$ be Jordan-orthogonal projections in $A$. In any composite AB, and for any $b \in B_{+}, p \otimes b$ and $q \otimes b$ are Jordan-orthogonal.

Proof: Since $\otimes: A \times B \rightarrow A B$ is a positive bilinear map, we have for all $a_{1}, a_{2} \in A$ and all $b \in B$ that $a_{1} \leq a_{2} \Rightarrow a_{1} \otimes b \leq a_{2} \otimes b$ (since $a_{2} \otimes b-a_{1} \otimes b=\left(a_{2}-a_{1}\right) \otimes b$ is positive). Now suppose $b$ is an effect in $B$ and that $p$ and $q$ are Jordan orthogonal projections in $A$, so that $p+q \leq u_{A}$. It's enough to show that $p \otimes b+q \otimes b \leq u_{A B}$. But $p \otimes b+q \otimes b=(p+q) \otimes b$, and $p+q \leq u_{B}$, so we have $(p+q) \otimes b \leq u_{A} \otimes b \leq u_{A} \otimes u_{B}=u_{A B}$. Since the result holds for arbitrary effects $b$, it holds for arbitrary elements $b \in B$.

Proposition 4.7. For all $a \in A, b \in B, a \otimes u_{B}$ and $u_{A} \otimes b$ operator commute in $A B$.

Note that this would follow trivially from Proposition 4.3 if $A B$ were spanned by pure tensors, that is, if $A B$ is locally tomographic.

Proof: Suppose $p \in A$ and $q \in B$ are projections, and let $p^{\prime}=u_{A}-p$ and $q^{\prime}=u_{B}-q$. Then we have

$$
u_{A B}=u_{A} \otimes u_{B}=\left(p+p^{\prime}\right) \otimes\left(q+q^{\prime}\right)=p \otimes q+p^{\prime} \otimes q+p \otimes q^{\prime}+p^{\prime} \otimes q^{\prime} .
$$

By Proposition 4.5, the four terms on the right are projections. They are mutually orthogonal by Corollary 4.6, and sum to the unit in $A B$. Hence, $p \otimes u_{B}=p \otimes q+p \otimes q^{\prime}$ and $u_{A} \otimes q=p \otimes q+p^{\prime} \otimes q$ operator commute by [4], Lemma 1.48. Now let $a \in A$ and $b \in B$ be arbitrary: by the spectral theorem for EJAs, we have $a=\sum_{i} t_{i} p_{i}$ and $b=\sum_{j} s_{j} q_{j}$ for pairwise orthogonal families of projections $p_{i}$ and $q_{j}$ and scalars $t_{i}$ and $s_{j}$. Since $p_{i} \otimes u_{B}$ and $u_{A} \otimes q_{j}$ operator commute for all $i, j$, it follows that $a \otimes u_{B}=\sum_{i} t_{i} p_{i} \otimes u_{B}$ and $u_{A} \otimes b=\sum_{j} s_{j} u_{A} \otimes q_{j}$ also operator commute. 


\subsection{Composites of Direct Sums}

Let $A \simeq A_{0} \oplus A_{1}$. Then there is a natural homomorphism $G\left(A_{0}\right) \rightarrow G(A)$ given by $\phi \mapsto \phi \oplus \mathrm{id}_{A_{1}}$ (where $\left.\left(\phi \oplus \operatorname{id}_{A_{1}}\right)\left(A_{0}, a_{1}\right)=\left(\phi\left(A_{0}\right), a_{1}\right)\right)$. If $A B$ is a dynamical composite of $A$ and $B$, then let $A_{0} B=J\left(\pi\left(A_{0} \times B\right)\right)$, the Jordan subalgebra of $A B$ generated by pure tensors $A_{0} \otimes b$ with $A_{0} \in A_{0}$ (equipped with the inner product inherited from $A B)$. Note that we have a natural positive bilinear map $\pi_{0}: A_{0} \times B \rightarrow A_{0} B$, obtained by restricting and co-restricting $\pi: A \times B \rightarrow A B$ to $A_{0} \times B$ and $A_{0} B$.

The following two results, taken together, will be very helpful below

Proposition 4.8. $A_{0} B$ is a composite of $A_{0}$ and $B$.

Proof: We check the requirements of Definition 4.1. First, note that $u_{0} \otimes u_{B}$ is effective as the unit in $A_{0} B$. For all $a \in A_{0}$ and $b \in B$, we have $\left(u_{0} \otimes u_{B}\right) \bullet(a \otimes b)=\left(u_{0} \bullet a\right) \otimes b=a \otimes b$ by the fundamental identity and the fact that $u_{0}$ is the unit in $A_{0}$. Since this holds on all pure tensors in $\pi\left(A_{0} \times B\right)$, it holds throughout $A_{0} B$.

A normalized state $\alpha$ on $A_{0}$ extends in a canonical way to a normalized state $\bar{\alpha}$ on $A=A_{0} \oplus A_{1}$, given by $\bar{\alpha}\left(A_{0}, a_{1}\right)=\alpha\left(A_{0}\right)$. Thus, if $\beta$ is a normalized state on $B$, condition (c) in Definition 2.1 tells us that there is a normalized state $\gamma$ on $A B$ with $\gamma(a, b)=\bar{\alpha}(a) \beta(b)$ for all $a \in A$ and $b \in B$. Restricting $\gamma$ to $A_{0} B$ gives us a positive functional $\gamma_{0}$ with $\gamma_{0}\left(A_{0} \otimes b\right)=\alpha\left(A_{0}\right) \beta(b)$ for all $A_{0} \in A_{0}$ and $b \in B$. In particular, $\gamma_{0}\left(u_{0} \otimes u_{B}\right)=\alpha\left(u_{0}\right) \beta\left(u_{B}\right)=1$, so $\gamma_{0}$ is a normalized state on $A_{0} B$.

Embedding $G\left(A_{0}\right)$ in $G(A)$ in the obvious way (that is, $g \in G\left(A_{0}\right)$ acts on $\left(A_{0}, a_{1}\right)$ as $\left.\left(g A_{0}, a_{1}\right)\right)$, we have a homomorphism $\otimes_{0}: G\left(A_{0}\right) \times G(B) \rightarrow G\left(A_{0} B\right)$ obtained by restricting the domain of the given homomorphism $\otimes: G(A) \times G(B) \rightarrow G(A B)$. For any $h \in G(B)$ and any $A_{0} \in A, b \in B$ we have $(g \otimes h)\left(A_{0} \otimes b\right)=g A_{0} \otimes h b$. Since this last lies in $\pi\left(A_{0} \times B\right), g \otimes h$ fixes $A_{0} B$. Thus, we have a natural homomorphism $G\left(A_{0}\right) \times G(B) \rightarrow$ $G\left(A_{0} B\right)$ satisfying the requirements for a dynamical composite. Finally, note that $(g \otimes h)^{\dagger}=g^{\dagger} \otimes h^{\dagger}$ since this holds at the level of $A B$.

Recall that the center of an EJA $A$ is the set of all elements in $A$ that operator commute with each element of $A$. Such elements are called central. A central projection is thus a projection $p \in A$ such that $L_{p} \circ L_{a}=L_{a} \circ L_{p}$ for all $a \in A$.

Proposition 4.9. Let $A B$ be any composite of EJAs $A$ and $B$. If $A=\oplus_{i} A_{i}$ and $B=\bigoplus_{j} B_{j}$ where $A_{i}=c_{i} \bullet A$ and $B_{j}=d_{j} \bullet B$ for mutually Jordan orthogonal families of central projections $c_{i}$ in $A$ and $d_{j}$ in $B$, then $A B=\bigoplus_{i, j} A_{i} B_{j}$.

Proof: It is enough to prove this for the case in which $A=c \bullet A \oplus c^{\prime} \bullet A$ for a central idempotent $c$, where $c^{\prime}=u_{A}-c$. By ([4], Lemma 1.43), if $p$ is any idempotent in an EJA $A$, then $U_{p}(A)$ is a Jordan subalgebra of $A$. Moreover, if $p$ and $q$ are Jordan-orthogonal idempotents, then $U_{p}(A)$ is Jordan-orthogonal to $U_{p}(B)$, by ([4], Lemma 1.45). Now let $c$ be a central projection in $A$, and write $A_{c}$ for $c \bullet A$. Then $c \otimes u_{B}$ is an idempotent in $A B$, and Corollary 4.4 gives us

$$
x \otimes y=(c \bullet x) \otimes y=U_{c}(x) \otimes y=U_{c \otimes y}(x \otimes y) \in U_{c \otimes y}(A B) .
$$

Accepted in 〈 Xuantum 2020-10-07, click title to verify. Published under CC-BY 4.0. 
for all $x \in A_{c}$ and $y \in B$. As pure tensors $x \otimes y$ with $x \in A_{c}$ and $y \in B$ generate $A_{c} B$, we now have $A_{c} B \leq U_{c \otimes i d_{B}}(A B)$. By the same token $A_{c^{\prime}} B \leq U_{c^{\prime} \otimes i_{B}}(A B)$. As noted above, the two larger subalgebras are pairwise Jordan-orthogonal; hence, so are the two smaller ones. In particular, the (internal) direct sum $A_{c} B \oplus A_{c^{\prime}} B$ exists, and is a Jordan subalgebra, of $A B$. Every pure tensor in $A \otimes B$ has the form $\left(x_{1}+x_{2}\right) \otimes y=x_{1} \otimes y+x_{2} \otimes y$, where $x_{1} \in A_{c}$ and $x_{2} \in A_{c^{\prime}}$, and hence, belongs to $A_{c} B \otimes A_{c^{\prime}} B$. Since such pure tensors generate $A B$ as a Jordan algebra, we have $A B=A_{c} B \oplus A_{c^{\prime}} B$. The general case now follows by an easy induction.

Corollary 4.10. Let $c$ be a projection in an EJA $A$, and let $A B$ be a composite of $A$ with an $E J A B$. Then $c \otimes u_{B}$ is central in $A B$ if $c$ is central in $A$.

\subsection{Composites of simple EJAs}

We now show that if $A$ and $B$ are nontrivial simple EJAs, then any composite $A B$ must be special, universally reversible, and an ideal (a direct summand) of the universal tensor product $A \widetilde{\otimes} B$. It follows that $A$ and $B$ must be special; that is, no nontrivial composite exists if either factor is $M_{3}(\mathbb{O})$. The rough idea is that, since $A$ and $B$ have rank at least two, the fact that products of distinguishable effects are distinguishable will yield at least four distinguishable effects in $A B$. If the latter were simple, this would be the end of the story; but we know from the case of universal tensor products (which we will ultimately show are dynamical composites in our sense) that composites can have nontrivial direct summands. Therefore, we need to work a bit harder, and show that every irreducible direct summand of $A B$ has rank at least 4 .

We will need some preliminaries. An element $s \in A$ is called a symmetry iff $s^{2}=u{ }^{15}$ In this case $U_{s}$ is a Jordan automorphism of $A$, with $U_{s}^{2}=$ id ([4], Prop. 2.34). Also note that $p:=\frac{1}{2}(s+u)$ is a projection, and, conversely, if $p$ is a projection, then $s:=2 p-u$ is a symmetry. Two projections $p, q \in A$ are exchanged by a symmetry $s \in A$ iff $U_{s}(p)=q$ (in which case, $p=U_{s}(q)$ ). More generally, $p$ and $q$ are equivalent iff there exists a finite sequence of symmetries $s_{1}, \ldots s_{\ell}$ with $q=\left(U_{s_{\ell}} \circ \cdots \circ U_{s_{1}}\right)(p)$. It will be important below that if $A$ and $B$ are simple, then any two atomic projections are exchanged by a symmetry ([31], Lemma 5.3.2).

Lemma 4.11. Let $s \in A$ be a symmetry exchanging projections $p_{1}, p_{2} \in A$, and let $t \in B$ be a symmetry exchanging projections $q_{1}, q_{2} \in B$. Then $s \otimes u_{B}$ and $u_{A} \otimes t$ are symmetries in $A B$, and $U_{u_{A} \otimes t} U_{s \otimes u_{B}}\left(p_{1} \otimes q_{1}\right)=p_{2} \otimes q_{2}$. In particular, the projections $p_{1} \otimes q_{1}$ and $p_{2} \otimes q_{2}$ are equivalent.

Proof: $\left(s \otimes u_{B}\right)^{2}=s^{2} \otimes u_{B}=u_{A} \otimes u_{B}=u_{A B}$ by Proposition 4.3. Similarly for $u_{A} \otimes t$. Now by Corollary 4.4, we have

$$
U_{u_{A} \otimes t} U_{s \otimes u_{B}}\left(p_{1} \otimes q_{1}\right)=U_{u_{A} \otimes t}\left(U_{s}\left(p_{1}\right) \otimes q_{1}\right)=U_{s}\left(p_{1}\right) \otimes U_{t}\left(q_{1}\right)=p_{2} \otimes q_{2} .
$$

\footnotetext{
${ }^{15}$ Not to be confused with a symmetry qua order-automorphism.
} 
Theorem 4.12. Let $A B$ be a composite of simple, nontrivial Jordan algebras $A$ and $B$. Then $A B$ is a special, universally reversible EJA.

Proof: We shall show that every irreducible direct summand of $A B$ has rank $\geq 4$, from which the result follows. Decompose $A B$ as a direct sum of simple ideals, say $A B=$ $\bigoplus_{\alpha} M_{\alpha}$. Let $\pi_{\alpha}: A B \rightarrow M_{\alpha}$ be the corresponding projections, and let $u_{\alpha}:=\pi_{\alpha}\left(u_{A B}\right)$ be the unit in $M_{\alpha}$. Suppose now that $\left\{p_{1}, \ldots, p_{n}\right\}$ is a Jordan frame in $A$ and $\left\{q_{1}, \ldots, q_{m}\right\}$ is a Jordan frame in $B$. By Proposition 4.5 and Corollary $4.6, p_{i} \otimes p_{j}$ are pairwise orthogonal projections in $A B$. Since $A$ and $B$ are simple, there are symmetries in $A B$ exchanging the $p_{i}$, and there are symmetries in $B$ exchanging the $q_{j}$. By Lemma 4.11, therefore, the projections $p_{i} \otimes q_{j}$ are pairwise equivalent. By [4] Lemma 3.9, therefore, these projections have the same central cover $c$. This means that for each $\alpha$, the projection $\pi_{\alpha}: A B \rightarrow M_{\alpha}$ takes none of the projections $p_{i} \otimes q_{j}$ to the zero projection in $M_{\alpha}$, or it takes all of them to zero - the former case arising exactly when $u_{\alpha} \leq c$, and the latter, when $u_{\alpha} c=c u_{\alpha}=0$. If $M_{\alpha}$ is of the first type, $\left\{\pi_{\alpha}\left(p_{i} \otimes q_{j}\right) \mid i=1, \ldots, n, j=1, \ldots, m\right\}$ consists of $\mathrm{nm}$ distinct orthogonal projections in $M_{\alpha}$, summing to the unit $\pi_{\alpha}(u)=: u_{\alpha}$. Hence, the rank of $M_{\alpha}$ is at least $n m$. In particular, since $A$ and $B$ are nontrivial, $n, m \geq 2$, whence, $M_{\alpha}$ has rank at least 4 , and hence, is special.

Now let $p, q$ be arbitrary projections in $A$ and $B$, respectively: extending each to a Jordan frame, as above, we see that for all $\alpha$, if $\pi_{\alpha}(p \otimes q) \neq 0$, then $M_{\alpha}$ is special. Hence, $p \otimes q$ belongs to the direct sum of the special summands of $A B$, i.e., to $M_{\mathrm{sp}}$. Since projections $p \otimes q$ generate $A B$, the latter is special.

The argument also shows that each simple direct summand $M_{\alpha}$, in addition to being special, is not a spin factor, and hence, is UR. Since direct sums of universally reversible EJAs are again UR (Appendix A, Proposition A.7), it follows that $A B$ must be UR.

Proposition 4.13. Let $A$ and $B$ be EJAs having no 1-dimensional summands. If $A B$ is a composite of $A$ and $B$, then $A B$ is $U R$.

Proof: Decompose $A$ and $B$ into simple direct summands, say $A=\bigoplus_{i} A_{i}$ and $B=\bigoplus B_{j}$. By Proposition 4.9, $A B=\bigoplus A_{i} B_{j}$. By Proposition 4.8, each summand $A_{i} B_{j}$ is a composite, and hence, by the preceding result, UR. But direct sums of universally reversible EJAs are again UR.

We call an ideal trivial if it is isomorphic to a direct sum of rank-one EJAs.

Proposition 4.14. If $A$ contains an exceptional ideal and $B$ contains a nontrivial ideal, there exists no composite $A B$ satisfying the conditions of Definition 1.

Proof: Suppose $B$ has a nontrivial ideal $B_{1}$, and that a composite $A B$ exists. Let $A_{0}$ be any simple ideal in $A$. By Proposition $4.8, A_{0} B$ is a composite of $A_{0}$ and $B$. Let $B=B_{0} \oplus B_{1}$ where $B_{1}$ is a nontrivial simple ideal of $B$. Then, applying Proposition 4.8 again, $A_{0} B_{1}=J\left(A_{0} \otimes B_{1}\right) \leq A_{0} B$, is a composite. Since $A_{0}$ and $B_{1}$ are simple, Theorem 4.12 implies that $A_{0} B_{1}$ is special. The fundamental identity (1) implies that 
the mapping $A_{0} \rightarrow A_{0} B_{1}$, given by $a \mapsto a \otimes u_{1}$, where $u_{1}$ is the unit of $B_{1}$, is a faithful Jordan homomorphism. Therefore, $A_{0}$ is special.

Another way to express Proposition 4.14 is that if $A$ is exceptional and $A B$ exists, then $B$ must be a direct sum of 1-dimensional EJAs - in other words, a classical system.

Theorem 4.15. Let $A$ and $B$ be simple, special EJAs. Then $A B$ is an ideal in $A \widetilde{\otimes} B$.

Proof: By Propositions 4.3 and 4.7, we have Jordan homomorphisms $A, B \rightarrow A B$ with operator-commuting ranges. Since $A B$ is special, i.e., a JC-algebra, elements of $A B$ operator commute iff their images in $C^{*}(A B)$ operator commute ([30], Lemma 5.1). Thus, we have Jordan homomorphisms $A, B \rightarrow C^{*}(A B)$ with operator-commuting ranges. The universal property given by Proposition 3.9 (a) of $A \widetilde{\otimes} B$ yields a Jordan homomorphism $\phi: A \widetilde{\otimes} B \rightarrow C^{*}(A B)$ taking (the image of) $a \otimes b$ in $A \widetilde{\otimes} B$ to (the image of $a \otimes b$ in $C^{*}(A B)$. Since both $A \widetilde{\otimes} B$ and $A B$ are generated by pure tensors, $\phi$ takes $A \widetilde{\otimes} B$ onto $A B$. Letting $K$ denote the kernel of $\phi$, an ideal of $A \widetilde{\otimes} B$, we have $A \widetilde{\otimes} B=K^{\perp} \oplus K$, where $K^{\perp}$ is the complementary ideal; the mapping $\phi$ factors through the projection $A \widetilde{\otimes} B \rightarrow K^{\perp}$ to give an isomorphism $\phi^{\prime}: K^{\perp} \simeq A B$, since the restriction $\phi^{\prime}$ of the surjection $\phi$ to $K^{\perp}$ is injective.

In appendix A, we show that $C^{*}(A \oplus B)=C^{*}(A) \oplus C^{*}(B)$ (Proposition A.6). Combining this fact with Proposition 4.9, we can extend the preceding result as follows.

Corollary 4.16. Let $A B$ be a composite of special EJAs $A$ and $B$. Then $A B$ is a direct summand of $A \widetilde{\otimes} B$.

Combined with Table 2, Theorem 4.15 sharply restricts the possibilities for composites of simple EJAS. In particular, it follows that if $A \widetilde{\otimes} B$ is itself simple, then $A B \simeq A \widetilde{\otimes} B$. In other words, in this case the universal tensor product is the only "reasonable" tensor product (to the extent that we think the conditions of Definition 4.1 constitute reasonableness, in this context). If $A=B=C_{n}$, so that $A \widetilde{\otimes} B=$ $M_{n^{2}}(\mathbb{C})_{s a} \oplus M_{n^{2}}(\mathbb{C})_{s a}$, we have another candidate, i.e., the usual quantum-mechanical composite $M_{n^{2}}(\mathbb{C})_{s a}$. If $A=B=M_{2}(\mathbb{H})$ (that is, if $A$ and $B$ are two quaternionic bits), we have $A \widetilde{\otimes} B=M_{16}(\mathbb{R})_{s a} \oplus M_{16}(\mathbb{R})_{s a} \oplus M_{16}(\mathbb{R})_{s a} \oplus M_{16}(\mathbb{R})_{s a}$, giving us four possibilities for $A B$. These exhaust the possibilities for composites of simple real, complex and quaternionic quantum systems!

\section{$5 \quad$ EJC-algebras}

In view of Theorem 4.12 and Corollary 4.14, we now restrict our attention to special EJAs. A JC-algebra is variously defined as a norm-closed Jordan subalgebra of $\mathcal{L}(\mathbf{H})$ for a real or complex Hilbert space $\mathbf{H}$, or as a Jordan algebra that is Jordan-isomorphic to such an algebra. In finite dimensions, any JC algebra is euclidean, and any special 
euclidean Jordan algebra is JC (on the second definition). Here, we consider EJAs that are embedded, not necessarily in $\mathcal{L}(\mathbf{H})$ for a specific Hilbert space, but in some definite complex *-algebra.

Definition 5.1. An embedded $J C$ algebra (EJC) is a triple $\left(A, \pi_{A}, \mathbf{M}_{A}\right)$ where $A$ is an EJA, $\mathbf{M}_{A}$ is a finite-dimensional complex $*$-algebra, and $\pi_{A}$ is a unital, injective Jordan homomorphism $A \rightarrow \mathbf{M}_{A_{s a}}$.

As the notation suggests, we often simply write $A$ for $\left(A, \pi_{A}, \mathbf{M}_{A}\right)$. Where no confusion is likely to arise, we shall usually identify $A$ with the Jordan subalgebra $\pi_{A}(A)$ of $\mathbf{M}_{A_{s a}}$, denoting $\left(A, \pi, \mathbf{M}_{A}\right)$ by the pair $\left(A, \mathbf{M}_{A}\right)$. For aesthetic reasons, we abbreviate the phrase "embedded euclidean JC" by EJC (rather than EEJC), letting the initial $E$ stand simultaneously for embedded and euclidean.

Notice that Definition 5.1 does not require that $A$ generate $\mathbf{M}_{A}$ as a complex *algebra. While most of our examples satisfy this condition, few of our general results depend on it.

In this section, we develop a canonical tensor product for EJC algebras, and use this to construct several symmetric monoidal categories of EJC-algebras. In one case, we obtain a category of reversible EJAs, with a monoidal product extending that of ordinary complex matrix algebras; in another, which we call InvQM, we restrict attention to EJAs that arise as fixed-point algebras of involutions on complex *-algebras (a class that includes all universally reversible EJCs, but also includes the quaternionic bit, $M_{2}(\mathbb{H})_{s a}$, as symplectically embedded in $\left.M_{4}(\mathbb{C})\right)$. Here, the monoidal structure agrees with the Hanche-Olsen tensor product in the cases in which the factors are UR.

For any *-algebra $\mathbf{M}$ and any set $S \subseteq \mathbf{M}_{s a}$, let $J(S)$ denote the Jordan subalgebra of $\mathbf{M}_{s a}$ generated by $S$. (We should probably write this as $J_{\mathbf{M}}(S)$, but context will generally make the usage unambiguous. See below for an example!)

Definition 5.2. The canonical tensor product of $\left(A, \mathbf{M}_{A}\right)$ and $\left(B, \mathbf{M}_{B}\right)$ is $\left(A \odot B, \mathbf{M}_{A} \otimes\right.$ $\left.\mathbf{M}_{B}\right)$, where $A \odot B:=J(A \otimes B) \subseteq \mathbf{M}_{A} \otimes \mathbf{M}_{B}$, the Jordan subalgebra of $\left(\mathbf{M}_{A} \otimes \mathbf{M}_{B}\right)_{s a}$ generated by $A \otimes B$. ${ }^{16}$

Note that this makes it a matter of definition that $\mathbf{M}_{A \odot B}=\mathbf{M}_{A} \otimes \mathbf{M}_{B}$. In particular, if $A$ and $B$ are universally embedded, so that $\mathbf{M}_{A}=C^{*}(A)$ and $\mathbf{M}_{B}=C^{*}(B)$, then $A \odot B=A \widetilde{\otimes} B$, so that $\mathbf{M}_{A \widetilde{\otimes} B}=C^{*}(A) \otimes C^{*}(B)$ by definition; but the fact that this last is $C^{*}(A \widetilde{\otimes} B)$ (whence, $A \odot B$ is UR) is a theorem. It will also be the case that $\odot$ preserves $C^{*}$-generation: if $A$ and $B$, as embedded, generate the $C^{*}$-algebras $\mathbf{M}_{A}$ and $\mathbf{M}_{B}$, then $A \odot B$ obviously generates $\mathbf{M}_{A \odot B}$.

Let us call an EJC $\left(A, \mathbf{M}_{A}\right)$ reversible iff $A$ is reversible in $\mathbf{M}_{A}$. Note that if $A$ is a simple EJC standardly embedded in $\mathbf{M}_{A}$, then $A$ is reversible iff $A=R_{n}, C_{n}$ or $Q_{n}$ for some $n$.

\footnotetext{
${ }^{16}$ When $\mathbf{M}_{A}=M_{n}(\mathbb{C})$ for some $n$ and similarly for $B$, this agrees with a construction by Jamjoom, Def. 2.1 in [38].
} 
We are going to show that the canonical composite of EJCs is a composite in the sense of Definition 4.1. In order to do so, we will need the following Lemma on extensions of derivations.

Lemma 5.3. Let $D$ be a derivation of an EJA $A$, and $\left(A, \mathbf{M}_{A}\right)$ a faithful representation of $A$. Then $D$ extends to a $*$-derivation of $\mathbf{M}_{A}$.

The proof can be found in Appendix E. ${ }^{17}$

Proposition 5.4. If $\left(A, \mathbf{M}_{A}\right)$ and $\left(B, \mathbf{M}_{B}\right)$ are EJC-algebras, then their canonical tensor product $A \odot B$ is a composite in the sense of Definition 4.1.

Proof: First we must show that $A \odot B$ is a dynamical composite, in the sense of Definition 2.3. In particular, it must be a composite of probabilistic models in the sense of Definition 2.1. Conditions (a)-(c) of that definition are easily verified: That $u_{A B}=u_{A} \otimes u_{B}$ follows from the unitality of the embeddings $A \mapsto \mathbf{M}_{A}, B \mapsto \mathbf{M}_{B}$ and $A B \mapsto \mathbf{M}_{A B}$. For all $a, x \in\left(\mathbf{M}_{A}\right)_{s a}$ and $b, y \in\left(\mathbf{M}_{B}\right)_{s a}$, we have

$$
\langle a \otimes b \mid x \otimes y\rangle=\operatorname{Tr}(a x \otimes b y)=\operatorname{Tr}(a x) \operatorname{Tr}(b y)=\langle a \mid x\rangle\langle b \mid y\rangle
$$

Thus, for any states $\alpha=\langle a|$ and $\beta=\langle b|$, where $a \in A_{+}$and $b \in B_{+}$, we have a state $\gamma=\langle a \otimes b|$ on $A B$ with $\gamma(x \otimes y)=\alpha(x) \beta(y)$ for all $x \in A_{+}, y \in B_{+}$.

That $A \odot B$ satisfies the additional conditions required to be a dynamical composite in the sense of Definition 2.3 is not trivial. Using Lemma 5.3 one can show that any $\phi \in G(A)$ extends to an element $\widehat{\phi} \in G\left(\left(\mathbf{M}_{A}\right)_{s a}\right)$ that preserves $A$, and that $\widehat{\phi} \otimes \mathbf{1}_{M_{B}}$ is an order-automorphism of $\left(\mathbf{M}_{A} \otimes \mathbf{M}_{B}\right)_{s a}$ preserving $A \odot B$. It follows that $\widehat{\phi} \otimes \widehat{\psi}=\left(\widehat{\phi} \otimes \mathbf{1}_{\mathbf{M}_{B}}\right) \circ\left(\mathbf{1}_{\mathbf{M}_{A}} \otimes \widehat{\psi}\right)$ preserves $A \odot B$ as well. The details are presented in Appendix E. It is not difficult to verify that $(\widehat{\phi} \otimes \widehat{\psi})^{\dagger}=\widehat{\phi}^{\dagger} \otimes \widehat{\psi}^{\dagger}$. Thus $A \odot B$ satisfies conditions (a) and (b) of Definition 4.1. Condition (c) is immediate from the definition of $A \odot B$.

Combining this with Theorem 4.12, we see that if $A$ and $B$ are simple reversible EJCs for which $A \widetilde{\otimes} B$ is also simple, then the canonical and universal tensor products of $A$ and $B$ coincide. This covers all cases except those involving two factors of the form $C_{n}$ and $C_{k}$, and those involving $Q_{2}$ as a factor. In fact, many of the latter are covered by the following.

Corollary 5.5. Let $\left(A, \mathbf{M}_{A}\right)$ and $\left(B, \mathbf{M}_{B}\right)$ be simple reversible EJCs with $A$ generating $M_{A}$ and $B$ generating $M_{B}$ as *-algebras. Suppose $M_{A}$ and $M_{B}$ carry involutions $\Phi$ and $\Psi$, respectively, with $\Phi$ fixing points of $A$ and $\Psi$ fixing points of $B$ (i.e. $A \subseteq \mathbf{M}_{A}^{\Phi}$ and $\left.B \subseteq \mathbf{M}_{B}^{\Phi}\right)$. Then $A \odot B=\left(\mathbf{M}_{A} \otimes \mathbf{M}_{B}\right)_{s a}^{\Phi \otimes \Psi}$, the set of self-adjoint fixed points of $\Phi \otimes \Psi$.

\footnotetext{
${ }^{17}$ In [61], Upmeier shows that every derivation of a reversible JC algebra $A$ acting on a complex Hilbert space $\mathcal{H}$ extends to a $*$-derivation of the $C^{*}$ algebra of operators on $\mathcal{H}$ generated by $A$. In finite dimensions, the hypothesis of reversibility is not needed. This is doubtless well known, but as we could find no reference to it, we have included a proof.
} 
Proof: By the preceding proposition, $A \odot B$ is a dynamical composite of $A$ and $B$; hence, by Theorem 4.12 and the simplicity of $A$ and $B, A \odot B$ is universally reversible. Since $\Phi \otimes \Psi$ fixes points of $A \otimes B$, it also fixes points of the Jordan algebra this generates in $M_{A} \otimes M_{B}$, i.e., of $A \odot B$. But then, by Proposition 3.7, $A \odot B$ is exactly the set of fixed points of $\Phi \otimes \Psi$.

For example, the quaternionic bit $Q_{2}$ standardly embedded in $M_{4}(\mathbb{C})=M_{2}\left(M_{2}(\mathbb{C})\right)$ as the set of block matrices of the form

$$
\left(\begin{array}{rr}
a & b \\
-\bar{b} & \bar{a}
\end{array}\right)
$$

with $a$ self-adjoint and $b$ antisymmetric is exactly the fixed-point set of the involution $\Phi(x)=-J_{2} x^{T} J_{2}$, where with $\mathbf{1}_{2}$ the $2 \times 2$ identity matrix

$$
J_{2}=\left(\begin{array}{cc}
0 & \mathbf{1}_{2} \\
-\mathbf{1}_{2} & 0
\end{array}\right)
$$

Thus, $Q_{2} \odot Q_{2}$ is the set of self-adjoint fixed points of $\Phi \otimes \Phi$ acting on $M_{4}(\mathbb{C}) \otimes M_{4}(\mathbb{C})=$ $M_{16}(\mathbb{C})$. From this, it follows that $Q_{2} \odot Q_{2} \simeq R_{16}=M_{16}(\mathbb{R})_{s a}$. For details, see Appendix B. Notice that Corollary 5.5 applies equally to all simple, universally embedded EJCs, and to all standardly embedded EJCs except for $C_{n}$ (recalling here that no (complexlinear) involution on $M_{n}(\mathbb{C})$ fixes all points of $\left.M_{n}(\mathbb{C})_{s a}\right)$.

Canonical composites of standardly embedded, reversible EJAs It follows from Proposition 5.4, together with Theorem 4.15 that the canonical and universal tensor products coincide for simple, reversible EJCs whose universal tensor products are simple. This covers all cases except for those in which one factor is $M_{2}(\mathbb{H})_{s a}$ (the quaternionic bit), and those in which both factors have the form $M_{n}(\mathbb{C})_{s a}$ for some $n$. Restricting attention to standardly embedded EJCs, these missing cases can be computed directly: see appendix B and [27]. The results are summarized, up to isomorphism, in the following table. (Only those products with a factor of $Q$ involve a nontrivial isomorphism; details of these will appear elsewhere.) Recall here the abbreviations $R_{n}=M_{n}(\mathbb{R})_{s a}, C_{n}=M_{n}(\mathbb{C})_{s a}$ and $Q_{n}=M_{n}(\mathbb{H})_{s a}$.

\begin{tabular}{c|ccc}
$\odot$ & $R_{n}$ & $C_{n}$ & $Q_{n}$ \\
\hline$R_{m}$ & $R_{m n}$ & $C_{m n}$ & $Q_{m n}$ \\
$C_{m}$ & $C_{m n}$ & $C_{m n}$ & $C_{2 m n}$ \\
$Q_{m}$ & $Q_{m n}$ & $C_{2 m n}$ & $R_{4 m n}$
\end{tabular}

Table 3: $\odot$, up to isomorphism, for standardly embedded, reversible EJCs

Associativity We now establish that the canonical tensor product is associative, setting the stage for the construction of symmetric monoidal categories of EJCs in Section 6. 
Proposition 5.6. $\odot$ is associative. More precisely, the associator mapping $\alpha: \mathbf{M}_{A} \otimes$ $\left(\mathbf{M}_{B} \otimes \mathbf{M}_{C}\right) \rightarrow\left(\mathbf{M}_{A} \otimes \mathbf{M}_{B}\right) \otimes \mathbf{M}_{C}$ carries $A \odot(B \odot C)$ isomorphically onto $(A \odot B) \odot C$.

The essence of the proof is the following Lemma.

Lemma 5.7. Let $\mathbf{M}$ and $\mathbf{N}$ be $*$-algebras, and let $A$ and $B$ be subspaces of $\mathbf{M}_{s a}$ and $\mathbf{N}_{s a}$, respectively, with $1=1_{\mathbf{M}} \in A$ and similarly for $B$. Then $J(A \otimes J(B))=J(A \otimes B)^{18}$.

Proof of Lemma 5.\%: We make use of the following notation. If $X$ and $Y$ are subsets (note: not necessarily subspaces) of $\mathbf{M}$ and $\mathbf{N}$, respectively, let

$$
X \bullet Y:=\{a \bullet b \mid a \in X, b \in Y\} ;
$$

similarly, $X+Y$ is the set of sums $a+b$ with $a \in X, b \in Y$, and, for $t \in \mathbb{R}, t X$ consists of multiples $t a$ of elements $a \in X$. Finally, we write $X \odot Y$ for the set $\{a \otimes b \mid a \in X, b \in Y\}$ of pure tensors of elements in $X$ and $Y$. Note that if $X$ and $Y$ happen to be subspaces of $\mathbf{M}$ and $\mathbf{N}$, respectively, then $J(X \odot Y)=J(X \otimes Y)$.

Now let $\mathcal{N}$ denote the set of all subsets $Y$ of $\mathbf{N}$ such that

(i) $B \subseteq Y \subseteq J(B)$, and

(ii) $A \odot Y \subseteq J(A \odot B)$.

Note that $B \in \mathcal{N}$; hence, $\mathcal{N}$ is non-empty. We are going to show first that $J(B)=\cup \mathcal{N}$.

Claim 1: If $Y_{1}, Y_{2} \in \mathcal{N}$, then the sets $Y_{1} \bullet Y_{2}, Y_{1}+Y_{2}$ and $t Y_{1}$ ( $t$ any real scalar) belong to $\mathcal{N}$.

To see this, suppose that $y_{1} \in Y_{1} \in \mathcal{N}$ and $y_{2} \in Y_{2} \in \mathcal{N}$. Then for any $a \in A$, we have

$$
\begin{aligned}
a \otimes\left(y_{1} \bullet y_{2}\right) & =a \otimes \frac{1}{2}\left(y_{1} y_{2}+y_{2} y_{1}\right) \\
& =\frac{1}{2}\left[\left(a \otimes y_{1} y_{2}\right)+\left(a \otimes y_{2} y_{1}\right)\right] \\
& =\frac{1}{2}\left[\left(a \otimes y_{1}\right)\left(1 \otimes y_{2}\right)+\left(1 \otimes y_{2}\right)\left(a \otimes y_{1}\right)\right] \\
& =\left(a \otimes y_{1}\right) \bullet\left(1 \otimes y_{2}\right) .
\end{aligned}
$$

Since $A \odot Y_{1}$ and $A \odot Y_{2}$ are both, by assumption, contained in $J(A \odot B)$, the last expression defines an element of $J(A \otimes B)$. Since $a$ is arbitrary here, all pure tensors from $A \odot\left(Y_{1} \bullet Y_{2}\right)$ belong to $J(A \odot B)$. Since the latter is a subspace of $(\mathbf{M} \otimes \mathbf{N})_{s a}$, it follows that linear combinations of pure tensors from $A \odot\left(N_{1} \bullet N_{2}\right)$ are contained in $J(A \odot B)$ as well. Finally, notice that $B \subseteq Y_{1}$ and $B \subseteq Y_{2}$ implies that $B \subseteq Y_{1} \bullet Y_{2}$ (for instance, express $b \in B$ as $b \bullet 1$ ). The corresponding claims for $Y_{1}+Y_{2}$ and $t Y_{1}$ are straightforward, since $a \otimes\left(y_{1}+y_{2}\right)=\left(a \otimes y_{1}\right)+\left(a \otimes y_{2}\right)$ and $a \otimes t y_{1}=t\left(a \otimes y_{1}\right)$.

\footnotetext{
${ }^{18}$ Note that on the left, $J(A \otimes J(B))$ refers to the Jordan subalgebra of $(A \otimes B)_{s a}$ generated by $A \otimes J(B)$, where $J(B)$ refers to the Jordan subalgebra of $\mathbf{N}_{s a}$ generated by $B$.
} 
Now let $J=\cup \mathcal{N}$. Claim 2: $J=J(B)$. Since $B \subseteq J \subseteq J(B)$, it's enough to show that $J$ is a Jordan subalgebra of $\mathbf{N}_{s a}$. Let $y_{1}, y_{2} \in J$ : then for some $Y_{1}, Y_{2} \in \mathcal{M}$, we have $y_{1} \in Y_{1}$ and $y_{2} \in Y_{2}$. By Claim $1, y_{1} \bullet y_{2} \in Y_{1} \bullet Y_{2} \in \mathcal{N}, y_{1}+y_{2} \in Y_{1}+Y_{2} \in \mathcal{N}$, and $t y_{1} \in t Y_{1} \in \mathcal{N}$; hence, $y_{1} \bullet y_{2}, y_{1}+y_{2}$ and $t y_{1}$ belong to $J$. In other words, $J$ is closed under scalar multiplication, addition and the Jordan product. This proves Claim 2.

We now use the fact that $J(B)=\bigcup \mathcal{N}$ to show that $J(A \otimes J(B))=J(A \otimes B)$. Let $\tau=\sum_{i} a_{i} \otimes y_{i}$ be an arbitrary element of $A \otimes J(B)$. Since $J(B)=\bigcup \mathcal{N}$, for each $i$, $y_{i} \in Y_{i}$ for some $Y_{i} \in \mathcal{N}$. But then, by definition of $\mathcal{N}$, for every $i a_{i} \otimes y_{i} \in A \odot Y_{i} \subseteq$ $J(A \otimes B)$; since the latter is a subspace of $\mathbf{M} \otimes \mathbf{N}, \tau \in J(A \otimes B)$ as well. Thus, $A \otimes J(B) \subseteq J(A \otimes B)$.

It now follows that $A \otimes B \subseteq A \otimes J(B) \subseteq J(A \otimes B)$, hence, that $J(A \otimes B) \subseteq$ $J(A \otimes J(B)) \subseteq J(A \otimes B)$.

Proof of Proposition 5.6: Let $\left(A, \mathbf{M}_{A}\right),\left(B, \mathbf{M}_{B}\right)$ and $\left(C, \mathbf{M}_{C}\right)$ be EJC-algebras. We need to show that the associator mapping $\alpha: \mathbf{M}_{A} \otimes\left(\mathbf{M}_{B} \otimes \mathbf{M}_{C}\right) \rightarrow\left(\mathbf{M}_{A} \otimes \mathbf{M}_{B}\right) \otimes \mathbf{M}_{C}$ carries $A \odot(B \odot C)$ onto $(A \odot B) \odot C$. Applying Lemma 5.6, (and noting that $A, B \odot C$, $A \odot B$ and $C$ all contain the relevant units), we have

$$
A \odot(B \odot C)=J(A \otimes J(B \otimes C))=J(A \otimes(B \otimes C))
$$

and

$$
(A \odot B) \odot C=J(J(A \otimes B) \otimes C)=J((A \otimes B) \otimes C) .
$$

The associator mapping is a *-isomorphism, and carries $A \otimes(B \otimes C)$ to $(A \otimes B) \otimes C$. Hence, it also carries $J(A \otimes(B \otimes C))$ onto $J((A \otimes B) \otimes C)$.

Direct sum of EJCs If $\left(A, \mathbf{M}_{A}\right)$ and $\left(B, \mathbf{M}_{B}\right)$ are two embedded EJAs, define $\left(A, \mathbf{M}_{A}\right) \oplus$ $\left(B, \mathbf{M}_{B}\right)=\left(A \oplus B, \mathbf{M}_{A} \oplus \mathbf{M}_{B}\right)$, where the embedding of $A \oplus B$ in $\mathbf{M}_{A} \oplus \mathbf{M}_{B}$ is the obvious one. Notice that by Proposition A.6, for universally embedded EJCs we have $\left(A, C^{*}(A)\right) \oplus\left(B, C^{*}(B)\right) \simeq\left(A \oplus B, C^{*}(A \oplus B)\right)$.

Lemma 5.8. For all EJCs $A, B$ and $C$, we have

$$
A \odot(B \oplus C)=(A \odot B) \oplus(A \odot C) .
$$

Proof: One can easily check that for sets $X \subseteq \mathbf{M}_{A}$ and $Y \subseteq \mathbf{M}_{B}, J(X \oplus Y)=$ $J(X) \oplus J(Y)$. Using this, and the distributivity of tensor products over direct sums in the contexts of vector spaces and $*$-algebras, we have

$$
\begin{aligned}
A \odot(B \oplus C)=J(A \otimes(B \oplus C)) & =J((A \otimes B) \oplus(A \otimes C)) \\
& =J(A \otimes B) \oplus J(A \otimes C) \\
& =(A \odot B) \oplus(A \odot C)
\end{aligned}
$$

(where $J$ refers variously to generated Jordan subalgebras of $\mathbf{M}_{A} \otimes\left(\mathbf{M}_{B} \oplus \mathbf{M}_{C}\right),\left(\mathbf{M}_{A} \otimes\right.$ $\left.\mathbf{M}_{B}\right) \oplus\left(\mathbf{M}_{A} \otimes \mathbf{M}_{C}\right), \mathbf{M}_{A} \otimes \mathbf{M}_{B}$, and $\left.\mathbf{M}_{A} \otimes \mathbf{M}_{C}\right)$. 


\section{Monoidal Categories of EJC-algebras}

As discussed in Section 2, a physical theory ought to be represented by a category, in which objects correspond to systems and morphisms to physical processes. An obvious candidate for a category in which objects are embedded JC-algebras is the following. Recall here that a linear map $\phi: \mathbf{M}_{A} \rightarrow \mathbf{M}_{B}$ is completely positive if the map $\phi:$ $M_{A} \otimes C_{n} \rightarrow M_{B} \otimes C_{n}$ is positive for each positive integer $n$.

Definition 6.1. Let $\left(A, \mathbf{M}_{A}\right)$ and $\left(B, \mathbf{M}_{B}\right)$ be EJC-algebras. A linear mapping $\phi$ : $\mathbf{M}_{A} \rightarrow \mathbf{M}_{B}$ is Jordan preserving iff $\phi(A) \subseteq B$. The category EJC has, as objects, EJC-algebras, and, as morphisms, completely positive Jordan-preserving mappings.

In view of Proposition 5.6, one might guess that EJC is symmetric-monoidal under $\odot$. There is certainly a natural choice for the monoidal unit, namely $I=(\mathbb{R}, \mathbb{C})$. However, the following examples show that tensor products of EJC morphisms are generally not morphisms.

Example 6.2. Let $\left(A, C^{*}(A)\right)$ and $\left(B, C^{*}(B)\right)$ be simple, nontrivial, universally embedded EJCs, and suppose that $B$ is not UR (e.g., the spin factor $V_{4}$ ). Suppose, further, that $A \widetilde{\otimes} B$ is irreducible - for instance, let $A=R_{n}$ for any $n$. Let $\widehat{B}$ be the set of fixed points of the canonical involution $\Phi_{B}$ (cf. Definition 3.6). Then by Theorem 4.15, $A \odot B=A \widetilde{\otimes} B$, the set of fixed points of $\Phi_{A} \otimes \Phi_{B}$. In particular, $u_{A} \otimes \widehat{B}$ is contained in $A \odot B$. Now let $\alpha$ be a state on $C^{*}(A)$. It is easily verified that every state is completely positive, ${ }^{19}$ and it is trivial that it is Jordan-preserving, and so, a morphism in EJC. But

$$
\left(\alpha \otimes \operatorname{id}_{B}\right)\left(u_{A} \otimes \widehat{B}\right)=\alpha\left(u_{A}\right) \widehat{B}=\widehat{B},
$$

which by Proposition 3.7 is larger than $B$ because $B$ is not UR. So $\alpha \otimes \mathrm{id}_{B}$ isn't Jordan-preserving.

The next example is similar:

Example 6.3. Let $C_{n}=M_{n}(\mathbb{C})_{s a}$ and $R_{k}=M_{k}(\mathbb{R})_{s a}$, as usual. Consider these as standardly embedded, i.e., consider the EJCs $\left(C_{n}, M_{n}(\mathbb{C})\right)$ and $\left(R_{k}, M_{k}(\mathbb{C})\right)$, where in the latter, $R_{k}$ is embedded as the set $k \times k$ complex matrices with real entries, i.e., the set of self-adjoint symmetric $k \times k$ complex matrices. Then we have

$$
\left(C_{n}, M_{n}(\mathbb{C})\right) \odot\left(R_{k}, M_{k}(\mathbb{C})\right)=\left(C_{n k}, M_{n k}(\mathbb{C})\right)
$$

where the embedding of $C_{n k}$ in $M_{n k}(\mathbb{C})$ is the standard one. Now let $\alpha$ be a state on $M_{n}(\mathbb{C})$, and let $b$ be any self-adjoint matrix in $M_{k}(\mathbb{C})$. Then $\mathbf{1}_{n} \otimes b$ is self-adjoint in $M_{n}(\mathbb{C}) \otimes M_{k}(\mathbb{C})=M_{n k}(\mathbb{C})$, and $\left(\alpha \otimes \operatorname{id}_{M_{k}}(\mathbb{C})\right)\left(\mathbf{1}_{n} \otimes b\right)=b$. Since $b$ needn't belong to $R_{k}$, the mapping $\alpha \otimes \operatorname{id}_{M_{k}}(\mathbb{C})$ isn't Jordan-preserving.

\footnotetext{
${ }^{19}$ Letting $\alpha$ be a state on $A$ and therefore on the complex matrix algebra $\mathbf{M}_{A}, \beta$ a positive functional on any complex matrix algebra $\mathbf{M}_{B}$, and $f \in\left(\mathbf{M}_{A} \otimes \mathbf{M}_{B}\right)_{+}$, we have $\beta\left(\left(\alpha \otimes \operatorname{id}{ }_{B}\right)(f)\right)=(\alpha \otimes \beta)(f)$. This is nonnegative since $\alpha \otimes \beta$ is a positive functional. Since this holds for all positive $\beta,\left(\alpha \otimes \operatorname{id}_{B}\right)(f)$ is positive, i.e. (since $\mathbf{M}_{B}$ was arbitrary) $\alpha$ is completely positive.
} 


\subsection{Completely Jordan Preserving Maps}

Examples 6.2 and 6.3 suggest the following adaptation of the notion of complete positivity to our setting.

Definition 6.4. A linear mapping $\phi:\left(A, \mathbf{M}_{A}\right) \rightarrow\left(B, \mathbf{M}_{B}\right)$ is completely Jordan preserving (CJP) iff, for any embedded JC-algebra $\left(C, \mathbf{M}_{C}\right)$, the mapping $\phi \otimes \mathrm{id}_{\mathbf{M}_{C}}$ is positive, and takes $A \odot C$ into $B \odot C$.

Note that this implies that $\phi$ is both Jordan-preserving (take $C=\mathbb{R}$ ) and completely positive (take $C=C_{n}$ for any $n$ ).

Lemma 6.5. If $\phi: \mathbf{M}_{A} \rightarrow \mathbf{M}_{B}$ is CJP, then for any $\left(C, \mathbf{M}_{C}\right), \phi \otimes i d_{\mathbf{M}_{C}}$ is again CJP.

Proof: If $\left(D, \mathbf{M}_{D}\right)$ is another embedded JC-algebra, consider $\left(C \odot D, \mathbf{M}_{C} \otimes \mathbf{M}_{D}\right)$. The associativity of $\odot$ gives us

$$
\left(\phi \otimes \operatorname{id}_{\mathbf{M}_{C}}\right) \otimes \operatorname{id}_{\mathbf{M}_{D}}=\phi \otimes\left(\operatorname{id}_{\mathbf{M}_{C} \otimes \mathbf{M}_{D}}\right) .
$$

Since $\phi$ is CJP, the latter carries $A \odot(C \odot D)$ into $B \odot(C \odot D)$. Since $\odot$ is associative, this tells us that $\left(\phi \otimes \mathrm{id}_{C}\right) \otimes \mathrm{id}_{D}$ carries $(A \odot C) \odot D$ into $(B \odot C) \odot D$.

While all CJP morphisms are CP, Example 6.2 shows that the converse is false. On the other hand, the class of CJP morphisms is still quite large.

Example 6.6. Let $\phi: \mathbf{M}_{A} \rightarrow \mathbf{M}_{B}$ be a $*$-homomorphism taking $A$ to $B$. It is easily verified that $\phi \otimes \mathrm{id}_{\mathbf{M}_{C}}$ is again a $*$-homomorphism. Since $*$-homomorphisms preserve the concrete Jordan product, ${ }^{20} \phi \otimes \mathrm{id}_{\mathbf{M}_{C}}$ takes the Jordan subalgebra generated by $A \otimes C$ to that generated by $B \otimes C$, i.e., sends $A \odot C$ into $B \odot C$. So $\phi$ is CJP.

Example 6.7. Let $\left(A, \mathbf{M}_{A}\right)$ be an embedded JC-algebra, and let $a \in A$. The mapping $U_{a}: \mathbf{M}_{A} \rightarrow \mathbf{M}_{A}$ given by $U_{a}(b)=a b a$ is completely positive; recall that its restriction to $\left(\mathbf{M}_{A}\right)_{s a}$ can be expressed in terms of the Jordan product on $\left(\mathbf{M}_{A}\right)_{s a}$ by

$$
U_{a}(b)=2 a \bullet(a \bullet b)-\left(a^{2}\right) \bullet b
$$

Since $A$ is a Jordan subalgebra of $\left(\mathbf{M}_{A}\right)_{s a}$, this makes it clear that $U_{a}(b) \in A$ for all $a, b \in A$. Thus, $U_{a}$ is a morphism. Now if $\left(C, \mathbf{M}_{C}\right)$ is another embedded JC-algebra, one easily sees $^{21}$ that

$$
U_{a} \otimes U_{c}=U_{a \otimes c}
$$

for all $c \in \mathbf{M}_{C}$; in particular,

$$
U_{a} \otimes \operatorname{id}_{\mathbf{M}_{C}}=U_{a} \otimes U_{\mathbf{1}}=U_{a \otimes \mathbf{1}} .
$$

Since $a \otimes \mathbf{1} \in A \odot C$, it follows that $U_{a}$ is CJP.

\footnotetext{
${ }^{20}$ That is, a $*$-homomorphism $\varphi$ satisfies $\varphi((x y+y x) / 2)=(\varphi(x) \varphi(y)+\varphi(y) \varphi(x)) / 2$.

${ }^{21}$ Observe that that $\left(U_{a} \otimes U_{c}\right)(x \otimes y)=a x a \otimes c y c=(a \otimes c)(x \otimes y)(a \otimes c)=U_{a \otimes c}(x \otimes y)$ and that the "pure tensors" $x \otimes y$ span $\mathbf{M}_{A} \otimes \mathbf{M}_{C}$.
} 
Proposition 6.8. Let $\left(A, \mathbf{M}_{A}\right),\left(A^{\prime}, \mathbf{M}_{A^{\prime}}\right),\left(B, \mathbf{M}_{B}\right),\left(B^{\prime}, \mathbf{M}_{B^{\prime}}\right)$ and and $\left(C, \mathbf{M}_{C}\right)$ be EJC-algebras.

(i) If $\phi: \mathbf{M}_{A} \rightarrow \mathbf{M}_{B}$ and $\psi: \mathbf{M}_{B} \rightarrow \mathbf{M}_{C}$ are CJP, then so is $\psi \circ \phi$, and

(ii) if $\phi: \mathbf{M}_{A} \rightarrow \mathbf{M}_{B}$ and $\psi: \mathbf{M}_{A^{\prime}} \rightarrow \mathbf{M}_{B^{\prime}}$ are CJP, then so is $\phi \otimes \psi: \mathbf{M}_{A \odot A^{\prime}}=$ $\mathbf{M}_{A} \otimes \mathbf{M}_{A^{\prime}} \rightarrow \mathbf{M}_{B} \otimes \mathbf{M}_{B^{\prime}}=\mathbf{M}_{B \odot B^{\prime}}$.

$$
\phi \otimes \psi=\left(\phi \otimes i d_{\mathbf{M}_{B^{\prime}}}\right) \circ\left(i d_{\mathbf{M}_{A}} \otimes \psi\right)
$$

is again CJP.

Proof: (i) is straightforward, and (ii) follows from (i) and Lemma 6.5.

As Example 6.2 illustrates, for certain non-UR EJCs $A$, there are no non-zero CJP maps from $A$ to $I$. In particular, normalized states on such an $A$ will not count as morphisms in the category CJP. Operationally, this means that the preparation of states and registration of effects need not correspond to any physical process in CJP. We now show that this defect can sometimes be remedied by relativizing the definition of CJP mappings to certain sub-categories of CJP.

\subsection{Relatively CJP Mappings}

Definition 6.9. Let $\left(A, \mathbf{M}_{A}\right)$ and $\left(B, \mathbf{M}_{B}\right)$ belong to $\mathcal{C}$. A positive linear mapping $\phi: \mathbf{M}_{A} \rightarrow \mathbf{M}_{B}$ is relatively $C J P$ with respect to $\mathcal{C}$ if, for all $\left(C, \mathbf{M}_{C}\right) \in \mathcal{C}$, the mapping $\phi \otimes \operatorname{id}_{\mathbf{M}_{C}}$ is positive and maps $A \odot C$ into $B \odot C$. We denote the set of all such maps by $\mathbf{C J P}_{\mathcal{C}}(A, B)$.

Proposition 6.10. Let $\mathcal{C}$ be any class of EJCs closed under the formation of the canonical tensor product and containing the trivial EJC I. Then $\mathcal{C}$ is a symmetric monoidal category with completely positive relatively CJP mappings as morphisms and the canonical tensor product as the monoidal product.

Proof: Exactly as in the proof of Proposition 6.8, we see that if $A, B, C \in \mathcal{C}$ and $\phi \in \mathbf{C J P}_{\mathcal{C}}(A, B)$ and $\psi \in \mathbf{C J P}_{\mathcal{C}}(B, C)$, then $\psi \circ \phi \in \mathbf{C J P}_{\mathcal{C}}(A, C)$, and also that if $A, B, C, D \in \mathcal{C}$ and $\phi \in \mathbf{C J P}_{\mathcal{C}}(A, B)$ and $\psi \in \mathbf{C J P}_{\mathcal{C}}(C, D)$, then $\phi \otimes \psi \in \mathbf{C J P}_{\mathcal{C}}(A \otimes$ $C, B \otimes D)$. In other words, $\mathcal{C}$ becomes a symmetric monoidal category with relatively CJP mappings as morphisms. We denote this category by $\mathbf{C J P}_{\mathcal{C}}$. Note that $\phi$ 's being relatively CJP with respect to $\mathcal{C}$ implies that $\phi$ is CP. For, either $\mathcal{C}$ contains an $A$ such that $\mathbf{M}_{A}$ is noncommutative, or it does not. If all $\mathbf{M}_{A}$ are commutative, then all positive maps between them are CP. On the other hand, if some $\mathbf{M}_{A}$ is noncommutative, it contains a summand isomorphic to $M_{n}(\mathbb{C})$ for some $n \geq 2$. By closure under $\odot$, it contains $C$ with a summand equal to $M_{m}(\mathbb{C})$ for arbitrarily large $m$ (just take $C=$ $A \odot A \odot \cdots \odot A$ with sufficiently many factors of $A$ ). Since the definition of "completely Jordan preserving relative to $\mathcal{C}$ " requires $\phi \otimes \mathrm{id}_{C}$ be positive for all $C$, and positivity on a matrix algebra requires positivity on all summands, we have that for every positive 
integer $n$, there exists $m \geq n$ such that $\phi \otimes M_{m}(\mathbb{C})$ is positive. That is sufficient for $\phi$ to be completely positive.

Example 6.11 (The category $\mathbb{C Q M}$ ). Let $\mathcal{C}$ be the class of hermitian parts of complex *-algebras with standard embeddings. Then $\phi$ belongs to $\mathrm{CJP}_{\mathcal{C}}$ iff $\phi$ is CP. Evidently, this category is essentially orthodox, mixed-state QM with superselection rules. From now on, we shall call this category $\mathbb{C} Q M$.

Example 6.12 (The category RSE). Recall that the standard embeddings for simple, reversible Jordan algebras represent

(a) $R_{n}$ as the set of self-adjoint elements of $M_{n}(\mathbb{C})$ having real entries;

(b) $C_{n}$ as itself, that is, the set of all self-adjoint elements of $M_{n}(\mathbb{C})$;

(c) $Q_{n}$ as the set of $2 n \times 2 n$ complex matrices having symplectic $2 \times 2$ block structure.

For $A=R_{n}, C_{n}$ or $Q_{n}$, let $\operatorname{St}(A)$ denote the matrix algebra, and $\sigma_{A}: A \rightarrow \operatorname{St}(A)$ the embedding, given above. Now let $\mathcal{C}_{o}$ denote the class of reversible, simple EJCs $\left(A, \mathbf{M}_{A}\right)$ that are standardly embedded up to isomorphism, in the sense that there exists a $*$-isomorphism $\phi: \mathbf{M}_{A} \simeq \operatorname{St}(A)$ acting on the identity on $A$ (or, more exactly, such that $\phi \circ \pi_{A}=\sigma_{A}$. ) It is straightforward to check that $\mathcal{C}_{o}$ is closed under the canonical tensor product [27]. Now let $\mathcal{C}$ consist of direct sums of EJCs belonging to $\mathcal{C}_{o}$. Since the canonical tensor product distributes over direct sums, by Lemma $5.8, \mathcal{C}$ is also closed under $\odot$. By the remarks above, then, $\mathbf{C J P}_{\mathcal{C}}$ is a symmetric monoidal category, which we call RSE. This represents a kind of unification of finite-dimensional real, complex and quaternionic quantum mechanics, in so far as its objects are the Jordan algebras associated with real, complex and quaternionic quantum systems, and direct sums of these. Moreover, as restricted to complex (or real) systems, its compositional structure is the standard one. However, Example 6.3 shows that not every quantummechanical process - in particular, not even processes that prepare states - will count as a morphism in RSE, so this unification comes at a high cost.

Example 6.13 (The category URUE). Let $\mathcal{C}$ be the class of universally reversible EJCs with universal embeddings. By Proposition 5.4 and Theorem 4.12, if $A$ and $B$ are simple, then $A \odot B$ is UR. It is straightforward that $C^{*}(A \oplus B)=C^{*}(A) \oplus C^{*}(B)$ (Appendix A, Proposition A.6) and that $A \oplus B$ is UR iff $A$ and $B$ are UR (Corollary A.7). Finally, we know that the canonical tensor product distributes over direct sums (Lemma 5.8). Putting these observations together, we see that $\mathcal{C}$ is closed under $\odot$. We denote the category $\mathbf{C J P}_{\mathcal{C}}$ by URUE.

Morphisms in URUE can be characterized more concretely: they are precisely those $\mathrm{CP}$ maps that intertwine the canonical involutions on universal $C^{*}$ algebras.

Proposition 6.14. Let $A$ and $B$ be UR, universally embedded EJCs. Then a mapping $\phi: C^{*}(A) \rightarrow C^{*}(B)$ belongs to URUE iff it is $C P$, and satisfies $\phi \circ \Phi_{A}=\Phi_{B} \circ \phi$. 
Proof: First, we show that CP intertwiners are relatively CJP. Let $\phi: A \rightarrow B$ be a $\mathrm{CP}$ intertwiner. We are to show that for every $C \in \mathcal{C}, \phi \otimes \operatorname{id}_{C}: A \odot C \rightarrow B \odot C$ is positive and Jordan-preserving. It is positive because $\phi$ is CP. By Corollary 5.5, we have $A \odot C=\left(\mathbf{M}_{A} \otimes \mathbf{M}_{C}\right)^{\Phi_{A} \otimes \Phi_{C}}$ and $B \odot C=\left(\mathbf{M}_{B} \otimes \mathbf{M}_{C}\right)^{\Phi_{B} \otimes \Phi_{C}}$. Intertwining then immediately gives that $\phi \otimes \mathrm{id}_{C}$ is Jordan-preserving: suppose $x \in A \odot C$, i.e. $\left(\Phi_{A} \otimes \Phi_{C}\right)(x)=x$. Then $\left(\Phi_{B} \otimes \Phi_{C}\right) \circ\left(\phi \otimes \operatorname{id}_{C}(x)\right)=\left(\left(\Phi_{B} \circ \phi\right) \otimes \Phi_{C}\right)(x)=\left(\phi \circ \Phi_{A}\right) \otimes$ $\left.\Phi_{C}\right)(x)=\left((\phi \otimes \mathrm{id}) \circ\left(\Phi_{A} \otimes \Phi_{C}\right)\right)(x)=\left(\phi \otimes \operatorname{id}_{C}\right)(x)$, i.e. $\left(\phi \otimes \operatorname{id}_{C}\right)(x)$ is fixed by $\Phi_{B} \otimes \Phi_{C}$, i.e. it is in $B \odot C$.

For the converse, suppose $\phi$ is relatively CJP for the class of UR, universally embedded EJCs. Note that $C^{*}(A)_{s a}$ decomposes as the direct sum of $\Phi_{A}$ 's +1 and -1 eigenspaces. Moreover, as $A$ is UR, the +1 eigenspace is precisely $A$. Now, $\phi \circ \Phi_{A}=\Phi_{B} \circ \phi$ trivially on $A$. We now claim that $\phi$ also intertwines $\Phi_{A}$ and $\Phi_{B}$ on the -1-eigenspace. To see this, note that $\phi_{A} \otimes \mathrm{id}_{C}$ is also relatively CJP for any object $C$ in URUE. If $a$ and $c$ belong to the -1-eigenspaces of $\Phi_{A}$ and $\Phi_{C}$, respectively, then $a \otimes c$ belongs to the +1 eigenspace of $\Phi_{A} \otimes \Phi_{C}$, whence,

$$
\left(\Phi_{B} \otimes \Phi_{C}\right)(\phi \otimes \mathrm{id})(a \otimes c)=(\phi \otimes \mathrm{id})\left(\Phi_{A} \otimes \Phi_{C}\right)(a \otimes c) .
$$

Since $\Phi_{C}(c)=-c$, this reduces to

$$
-\left(\Phi_{B}(\phi(a)) \otimes c\right)=-\left(\phi\left(\Phi_{A}(a)\right) \otimes c\right) .
$$

With $c$ non-zero, this implies that $\Phi_{B}(\phi(a))=\phi\left(\Phi_{A}(a)\right)$ for all $a$ in the -1 eigenspace of $\Phi_{A}$, as required. Thus, $\phi$ intertwines $\Phi_{A}$ and $\Phi_{B}$ on all of $C^{*}(A)_{s a}$, and hence, by the Cartesian decomposition, on all of $C^{*}(A)$.

As we will see below ( Corollary 6.17), maps in $\operatorname{URUE}(A, I)$ and $\operatorname{URUE}(I, A)$ correspond exactly to states and effects, respectively, on $A$. In this respect, the category URUE is closer than RSE to being a legitimate "unified" quantum theory, although it omits the quaternionic bit (which is reversible, but not universally so). Even as restricted to complex quantum systems, however, it differs from orthodox QM in two interesting ways. First, and most conspicuously, the tensor product is not the usual one: $C_{n} \widetilde{\otimes} C_{k}=C_{n k} \oplus C_{n k}$, rather than $C_{n k}$. Secondly, it allows some processes that orthodox QM does not. In particular, the mapping on $C^{*}\left(C_{n}\right)=M_{n}(\mathbb{C}) \oplus M_{n}(\mathbb{C})$ that swaps the two summands is a morphism in URUE. Since the image of $C_{n}$ in $C^{*}\left(C_{n}\right)$ consists of pairs $\left(a, a^{T}\right)$, this mapping effects the transpose automorphism on $C_{n}$. This is not permitted in orthodox QM, as the transpose is not a $\mathrm{CP}$ mapping on $M_{n}(\mathbb{C})$. (This causes no difficulty with positivity in the context of URUE, where the tensor product is different.)

In spite of its divergences from orthodoxy, URUE is in many respects a well-behaved probabilistic theory. Proposition 6.14 suggests a way in which it can be improved, which we explore in the next section. 


\subsection{The Category InvQM}

Recall that we write $\mathbf{M}^{\Phi}$ for the set of fixed-points of an involution $\Phi$ on a complex *algebra $\mathbf{M}$. Notice that the involution $\Phi$ on $M_{n}(\mathbb{C})$ with $R_{n}=M_{n}(\mathbb{C})_{s a}^{\Phi}$ (the transpose) and on $M_{2 n}(\mathbb{C})$ with $Q_{n}=M_{2 n}(\mathbb{C})_{s a}^{\Phi}$, namely $\Phi(a)=-J a^{T} J$ where $J$ is the unitary in Eq. (2), are both unitary with respect to the trace inner product $\langle a, b\rangle:=\operatorname{Tr}\left(a b^{*}\right)^{22}$. The canonical involution on $C^{*}\left(C_{n}\right)=M_{n}(\mathbb{C}) \oplus M_{n}(\mathbb{C})$, namely, the mapping $(a, b) \mapsto$ $\left(b^{T}, a^{T}\right)$, is likewise unitary.

Definition 6.15. Call an EJC $\left(A, \mathbf{M}_{A}\right)$ involutive iff there exists an involution $\Phi$ on $\mathbf{M}_{A}$, unitary with respect to the trace inner product on $\mathbf{M}_{A}$, with $A=\left(\mathbf{M}_{A}\right)_{s a}^{\Phi}$. Let InvQM denote the category in which objects are involutive EJC-algebras, and in which a morphism $\phi: A \rightarrow B$ is a CP mapping $\phi: \mathbf{M}_{A} \rightarrow \mathbf{M}_{B}$ intertwining $\Phi_{A}$ and $\Phi_{B}$, i.e, $\Phi_{B} \circ \phi=\phi \circ \Phi_{A}$.

As pointed out earlier, the condition that $A$ be the set of self-adjoint fixed points of an involution makes $A$ reversible in $\mathbf{M}_{A}$. Thus, the class of involutive EJC-algebras contains no "higher" $(n=4$ or $n>5)$ spin factors. In fact, up to isomorphism it contains exactly direct sums of the universally embedded UR EJCs $\left(A, C^{*}(A)\right)$ where $A=R_{n}, C_{n}$, with $n$ arbitrary, or $Q_{n}$ with $n>2$, together with the standardly embedded quabit, i.e., $\left(Q_{2}, M_{4}(\mathbb{C})\right)$, with $Q_{2}$ the self-adjoint fixed points of the involution $\Phi(a)=-J a^{T} J$ discussed in Section 3.3. In other words, InvQM includes exclusively quantum systems over the three division algebras $\mathbb{R}, \mathbb{C}$ and $\mathbb{H}$, albeit with the complex systems represented in their universal embeddings. Note that $\left(\mathbb{R}, M_{1}(\mathbb{R})\right)$ counts as an involutive EJC: since $M_{1}(\mathbb{R})=\mathbb{R}$ is commutative, the identity map provides the necessary involution.

Proposition 6.14 tells us that URUE is a full subcategory of InvQM, obtained by omitting the quabit. On the other hand, it is easy to see that CP mappings $\mathbf{M}_{A} \rightarrow \mathbf{M}_{B}$ intertwining $\Phi_{A}$ and $\Phi_{B}$ are automatically relatively CJP for the class of involutive EJCs. By Corollary 5.5 the canonical tensor product of involutive EJCs is again involutive, as

$$
A \odot B=\left(\mathbf{M}_{A} \otimes \mathbf{M}_{B}\right)_{s a}^{\Phi_{A} \otimes \Phi_{B}}
$$

for all $A, B \in \mathbf{I n v Q M}$. Proposition 5.3 implies that $A \odot B$ is a composite of $A$ and $B$ in the sense of Definition 4.1. Composites and tensor products of intertwining $\mathrm{CP}$ maps are also intertwining CP-maps (as are associators, unit-introductions and the swap mapping), so InvQM is a symmetric monoidal category - indeed, a monoidal subcategory of the category of involutive EJC-algebras and relatively CJP maps. In fact, these two categories are equal, because every step of the proof of Proposition 6.14 is valid for InvQM, indeed, for any category of the form $\mathbf{C J P}_{\mathcal{C}}$, where $\mathcal{C}$ is an $\odot$-closed subset of (the set of objects of) InvQM. So we have:

\footnotetext{
${ }^{22}$ We follow the convention that inner products are linear in the first argument.
} 
Proposition 6.16. Let $\mathcal{C}$ be any set of involutive EJCs closed under $\odot$. Then $\mathbf{C J P}_{\mathcal{C}}$ is a symmetric monoidal category, with hom-sets $\mathbf{C J P}_{\mathcal{C}}(A, B)=\left\{\phi: \mathbf{M}_{A} \rightarrow \mathbf{M}_{B}\right.$ : $\phi$ is completely positive and $\left.\phi \circ \Phi_{A}=\Phi_{B} \circ \phi\right\}$.

In other words, $\mathbf{C J P}_{\mathcal{C}}$ for such a $\mathcal{C}$ is a full monoidal subcategory of InvQM. In particular, $\mathbf{I n v Q M}=\mathbf{C J P}_{\text {obInvQM }}$.

In the special case in which $A$ and $B$ are universally embedded complex quantum systems, say $A=C_{n}$ and $B=C_{k}$, we have $\mathbf{M}_{A}=C^{*}\left(C_{n}\right)=M_{n}(\mathbb{C}) \oplus M_{n}(\mathbb{C})$ and similarly $\mathbf{M}_{B}=M_{k}(\mathbb{C}) \oplus M_{k}(\mathbb{C})$. The involutions $\Phi_{A}$ are given by $\Phi_{A}(a, b)=\left(b^{T}, a^{T}\right)$, and similarly for $\Phi_{B}$. In this case, the interwining CP-maps are sums of mappings of the two forms: $(a, b) \mapsto\left(\phi(a), \phi^{T}(b)\right)$ and $(a, b) \mapsto\left(\phi^{T}(b), \phi(a)\right)$, where $\phi$ is a CP mapping $M_{n}(\mathbb{C}) \rightarrow M_{k}(\mathbb{C})$ and $\phi^{T}$ is determined by the condition $\phi^{T}\left(x^{T}\right)=(\phi(x))^{T}$, i.e. $\phi^{T}:=T \circ \phi \circ T$.

Two special cases of InvQM-morphisms are worth emphasising:

Corollary 6.17. Let $\left(A, \mathbf{M}_{A}\right)$ belong to InvQM. Then

(a) $\operatorname{InvQM}(I, A)$ is the set of linear mappings $\phi_{a}: \mathbb{R} \rightarrow \mathbf{M}_{A}$, for each $a \in A_{+}$, where $\phi_{a}$ is the map determined by $\phi_{a}(1)=a$;

(b) $\operatorname{InvQM}(A, I)$ is the set of positive linear functionals (including, in particular, every state) on $\mathbf{M}_{A}$ of the form $|a\rangle, a \in A_{+}$.

(c) Each of $\operatorname{InvQM}(I, A)$ and $\operatorname{InvQM}(A, I)$ is a cone affinely isomorphic to $A_{+}$.

Proof: A linear map $\phi: I \rightarrow M_{A}$ is determined by $\phi(1)$. Let $\phi_{a} \in \operatorname{InvQM}(I, A)$ be the map with $\phi(1)=a$. Complete positivity of $\phi_{a}$ is equivalent to $a \in\left(\mathbf{M}_{A}\right)_{+},{ }^{23}$ and intertwining, to the condition that $a \in A .{ }^{24}$ So $a \in\left(\mathbf{M}_{A}\right)_{+} \cap A$, which by Proposition 3.2 is equal to $A_{+}$.

The completely positive maps $\mathbf{M}_{A} \rightarrow \mathbf{M}_{I}$ are the maps $|a\rangle, a \in\left(\mathbf{M}_{A}\right)_{+}$, i.e. $x \mapsto\langle x \mid a\rangle$, where the inner product is the canonical one on $\mathbf{M}_{A}$. (In particular, $a$ is Hermitian.) Recall that $\Phi_{I}=\mathrm{id}_{\mathbb{C}}$ so intertwining, for such a map, means that $\left\langle\Phi_{A}(x) \mid a\right\rangle=\langle x \mid a\rangle$. Unitarity of $\Phi_{A}$ implies $\left\langle\Phi_{A}(x), a\right\rangle=\left\langle x, \Phi_{A}(a)\right\rangle$, so intertwining, for Hermitian $x$, becomes $\left\langle x, \Phi_{A}(a)\right\rangle=\langle x, a\rangle$, i.e. $\Phi_{A}(a)=a$, i.e. $a \in A$. So we have established that $a \in\left(\mathbf{M}_{A}\right)_{+} \cap A=A_{+}$.

Considered as maps $A \rightarrow \mathcal{L}\left(\mathbb{R},\left(\mathbf{M}_{A}\right)_{s a}\right)$ and $\mathcal{L}\left(\left(\mathbf{M}_{A}\right)_{s a}, \mathbb{R}\right)$, the maps $\gamma: a \mapsto \phi_{a}$ and $\mu: a \mapsto|a\rangle$ induce isomorphisms of linear spaces between $A$ and their ranges, taking $A_{+}$onto $\operatorname{InvQM}(I, A)$ and $\operatorname{InvQM}(A, I)$ respectively, which establishes the

\footnotetext{
${ }^{23}$ Complete positivity certainly implies $a \in\left(\mathbf{M}_{A}\right)_{+}$, since positivity is equivalent to $a \in\left(\mathbf{M}_{A}\right)_{+}$. On the other hand, for any $C, \phi_{a} \otimes \operatorname{id}_{C}: \mathbf{M}_{I} \otimes \mathbf{M}_{C} \rightarrow \mathbf{M}_{A} \otimes \mathbf{M}_{C}$ acting on $x \in\left(\mathbf{M}_{I} \otimes \mathbf{M}_{C}\right)_{+} \simeq\left(\mathbf{M}_{C}\right)_{+}$ just gives $a \otimes x$, which is positive iff $a \in\left(\mathbf{M}_{A}\right)_{+}$.

${ }^{24}$ Noting that $\Phi_{I}=\mathrm{id}: \mathbf{M}_{I} \rightarrow \mathbf{M}_{I}$, we see that $\phi_{a} \circ \Phi_{I}=\phi_{a}$, so intertwining says that $\Phi_{A} \circ \phi_{a}=\phi_{a}$. Since a CP map intertwines iff it does so on the Hermitian part of its domain, intertwining is equivalent to $\Phi_{A} \circ \phi_{a}(\lambda)=\phi_{a}(\lambda)$ holding for all $\lambda \in \mathbb{R}$, i.e. $\lambda \Phi_{A}(a)=\lambda a$, which by involutiveness is equivalent to $a \in A$.
} 
affine isomorphisms claimed in (c).

The category InvQM provides a unification of finite-dimensional real, complex and quaternionic quantum mechanics, but with the same important caveats that apply to URUE: the representation of orthodox, complex quantum systems $C_{n}$ in InvQM is through the universal embedding $\psi: C_{n} \mapsto C^{*}\left(C_{n}\right)=M_{n}(\mathbb{C}) \oplus M_{n}(\mathbb{C}), a \mapsto\left(a, a^{T}\right)$. As a consequence, the composite of two complex quantum systems in InvQM is a direct sum of two copies of the their standard composite - equivalently, is the standard composite, combined with a classical bit. Moreover, the mapping that swaps the direct summands of $C^{*}\left(C_{n}\right)$, a perfectly good morphism in InvQM, acts as the transpose on $\psi(a)=\left(a, a^{T}\right)$.

\subsection{Compact Closure}

A compact structure on a symmetric monoidal category $\mathcal{C}$ is a choice, for every object $A \in \mathcal{C}$, of a dual object: A triple $\left(A^{\prime}, \eta_{A}, f_{A}\right)$ consisting of an object $A^{\prime} \in \mathbb{C}$, a co-unit $\eta_{A}: A \otimes A^{\prime} \rightarrow I$ and a unit $f_{A}: I \rightarrow A^{\prime} \otimes A$ such that

$$
A \rightarrow A \otimes I \stackrel{\mathrm{id}_{A} \otimes f_{A}}{\longrightarrow} A \otimes\left(A^{\prime} \otimes A\right) \longrightarrow\left(A \otimes A^{\prime}\right) \otimes A \stackrel{\eta_{A} \otimes \mathrm{id}_{A}}{\longrightarrow} I \otimes A \rightarrow A
$$

and

$$
A^{\prime} \rightarrow I \otimes A^{\prime} \stackrel{f_{A} \otimes \mathrm{id}_{A^{\prime}}}{\longrightarrow}\left(A^{\prime} \otimes A\right) \otimes A^{\prime} \longrightarrow A^{\prime} \otimes\left(A \otimes A^{\prime}\right) \stackrel{\mathrm{id}_{A^{\prime}} \otimes \eta_{A}}{\longrightarrow} A^{\prime} \otimes I \rightarrow A^{\prime}
$$

give the identity morphisms on $A$ and $A^{\prime}$, respectively (and where the unlabeled arrows are the obvious unit introductions and associators). ${ }^{25}$ The standard example is the category FinVec of finite-dimensional vector spaces (say, over $\mathbb{C}$ ) and linear mappings. Here there is a canonical linear functional $\eta_{A}: A \otimes A^{*} \rightarrow \mathbb{C}$, namely, the trace. A canonical unit is supplied by picking a basis $E$ for $A$, and setting $f_{A}=\sum_{x \in E} f_{x} \otimes f_{x}$, where $\left\{f_{x}\right\}$ is the dual basis for $A^{*}$; one then shows that this is independent of $E$, and that the identities above hold.

We say a symmetric monoidal category is compact closed if it admits a compact structure. ${ }^{26}$ In [1], it is shown that a large number of information-processing protocols, including in particular conclusive teleportation and entanglement-swapping, hold in any compact closed symmetric monoidal category, if we interpret objects as systems and morphisms as physically allowed processes. In this section, we shall see that our

\footnotetext{
${ }^{25}$ Our usage is slightly perverse. The usual convention is to denote the unit by $\eta_{A}$ and the co-unit by $\epsilon_{A}$. Our choice is motivated in part by the desire to represent states as morphisms $A \rightarrow I$ and effects as morphisms $I \rightarrow A$, rather than the reverse, together with the convention that takes the unit to correspond to the maximally entangled state.

${ }^{26}$ This makes compact closure a property of some symmetric monoidal categories (SMCs). Some authors instead define a compact closed category as a distinct mathematical structure, namely an SMC equipped with a distinguished compact structure.
} 
category InvQM is compact closed. More exactly, we shall show that it inherits a compact structure from the natural compact structure on the category $*$-Alg of finitedimensional complex $*$-algebras, which we now review.

If $\mathbf{M}$ is a finite-dimensional complex *-algebra, let $\operatorname{Tr}$ denote the canonical trace on $\mathbf{M}$, regarded as acting on itself by left multiplication (so that $\operatorname{Tr}(a)=\operatorname{tr}\left(L_{a}\right)$, $L_{a}: \mathbf{M} \rightarrow \mathbf{M}$ being $L_{a}(b)=a b$ for all $\left.b \in \mathbf{M}\right)$. This induces an inner product on $\mathbf{M}$, given by $\langle a, b\rangle_{\mathbf{M}}=\operatorname{Tr}\left(a b^{*}\right)^{27}$. Note that this inner product is self-dualizing, i.e,. $a \in \mathbf{M}_{+}$iff $\langle a, b\rangle \geq 0$ for all $b \in \mathbf{M}_{+}$.

Now let $\overline{\mathbf{M}}$ be the conjugate algebra, writing $\bar{a}$ for $a \in \mathbf{M}$ when regarded as belonging to $\overline{\mathbf{M}}$ (so that $\overline{c a}=\bar{c} \bar{a}$ for scalars $c \in \mathbb{C}$ and $\bar{a} \bar{b}=\overline{a b}$ for $a, b \in \mathbf{M}$ ). Note that $\langle\bar{a}, \bar{b}\rangle=\langle b, a\rangle$. Now define

$$
f_{\mathbf{M}}=\sum_{e \in E} e \otimes \bar{e} \in \mathbf{M} \otimes \overline{\mathbf{M}}
$$

where $E$ is any orthonormal basis for $\mathbf{M}$ with respect to $\langle\mid\rangle_{\mathbf{M}}$. Then straightforward computations show that $f_{\mathbf{M}} \in(\mathbf{M} \otimes \overline{\mathbf{M}})_{+}$, and that, for all $a, b \in \mathbf{M}$,

$$
\left\langle a \otimes \bar{b}, f_{\mathbf{M}}\right\rangle=\langle a, b\rangle=\operatorname{Tr}\left(a b^{*}\right)
$$

where the inner product on the left is the trace inner product on $\mathbf{M} \otimes \mathbf{M}^{*}$. Now define $\eta_{\mathbf{M}}: \mathbf{M} \otimes \overline{\mathbf{M}} \rightarrow \mathbb{C}$ by $\eta_{\mathbf{M}}=\left|f_{\mathbf{M}}\right\rangle$, noting that this functional is positive (so, up to normalization, a state) since $f_{\mathbf{M}}$ is positive in $\mathbf{M} \otimes \overline{\mathbf{M}}$.

A final computation shows that, for any states $\alpha$ and $\bar{\alpha}$ on $\mathbf{M}$ and $\overline{\mathbf{M}}$, respectively, and any $a \in \mathbf{M}, \bar{a} \in \overline{\mathbf{M}}$, we have

$$
\left(\eta_{\mathbf{M}} \otimes \alpha\right)\left(a \otimes f_{\overline{\mathbf{M}}}\right)=\alpha(a) \text { and }\left(\bar{\alpha} \otimes \eta_{\mathbf{M}}\right)\left(f_{\bar{M}} \otimes \bar{a}\right)=\bar{\alpha}(\bar{a})
$$

Thus, $\eta_{\mathbf{M}}$ and $f_{\overline{\mathbf{M}}}$ define a compact structure on $*-\mathbf{A l g}$, for which the dual object of $\mathbf{M}$ is given by $\overline{\mathbf{M}}$.

Definition 6.18. The conjugate of a EJC-algebra $\left(A, \mathbf{M}_{A}\right)$ is $\left(\bar{A}, \overline{\mathbf{M}}_{A}\right)$, where $\bar{A}=$ $\{\bar{a} \mid a \in A\}$. We write $\eta_{A}$ for $\eta_{\mathbf{M}_{A}}$ and $f_{A}$ for $f_{\mathbf{M}_{A}}$.

Any linear mapping $\phi: \mathbf{M} \rightarrow \mathbf{N}$ between $*$-algebras $\mathbf{M}$ and $\mathbf{N}$ gives rise to a linear mapping $\bar{\phi}: \overline{\mathbf{M}} \rightarrow \overline{\mathbf{N}}$, given by $\bar{\phi}(\bar{a})=\overline{\phi(a)}$ for $a \in \mathbf{M}$. It is straightforward to show that if $\Phi$ is a unitary involution on $\mathbf{M}_{A}$ with $A=\mathbf{M}_{A_{s a}}^{\Phi}$, then $\bar{\Phi}: \overline{\mathbf{M}} \rightarrow \overline{\mathbf{M}}$ is also a unitary involution with $\overline{\mathbf{M}}_{A_{s a}}^{\bar{\Phi}}=\bar{A}$. Thus, the class of involutive EJCs is closed under the formation of conjugates.

Lemma 6.19. Let $\left(A, \mathbf{M}_{A}\right)$ belong to InvQM. Then $f_{A} \in A \odot \bar{A}$.

Proof: By assumption, there is a unitary involution $\Phi$ on $\mathbf{M}_{A}$ such that $A=\left(\mathbf{M}_{A}\right)_{s a}^{\Phi}$; by Corollary 5.5, $A \odot \bar{A}$ is then the set of self-adjoint fixed points of $\Phi \otimes \bar{\Phi}$. Since $\Phi$ is unitary, if $E$ is an orthonormal basis for $\mathbf{M}_{A}$, then so is $\{\Phi(e) \mid e \in E\}$; hence, as $f_{A}$ is

${ }^{27}$ Again, we are following the convention that complex inner products are linear in the first argument. 
independent of the choice of orthonormal basis, $f_{A}$ is invariant under $\Phi \otimes \bar{\Phi}$. Since $f_{A}$ is also self-adjoint, it belongs to $\left(\mathbf{M}_{A} \otimes \mathbf{M}_{\bar{A}}\right)_{s a}^{\Phi \otimes \bar{\Phi}}$, i.e., to $A \odot \bar{A}$.

It follows now from part (b) of Corollary 6.17 that the functional

$$
\eta_{A}=\left|f_{\bar{A}}\right\rangle: \mathbf{M}_{\bar{A}} \otimes \mathbf{M}_{A} \rightarrow \mathbb{R}
$$

is an InvQM morphism. Hence, InvQM inherits the compact structure from $*-\mathbf{A l g}$, as promised. We have arrived at the following.

Theorem 6.20. InvQM is compact closed.

Dagger compactness In fact, we can do a bit better. A dagger on a category $\mathcal{C}$ is an involutive contravariant functor $\dagger: \mathcal{C} \rightarrow \mathcal{C}$ that fixes objects; that is, $A^{\dagger}=A$ for all $A \in \mathcal{C}$, and $f^{\dagger} \in \mathcal{C}(B, A)$ for all $f \in \mathcal{C}(A, B)$ with $f^{\dagger \dagger}=f$. If $\mathcal{C}$ is a symmetric monoidal category equipped with a dagger satisfying $(f \otimes g)^{\dagger}=f^{\dagger} \otimes g^{\dagger}$ for all morphisms $f$ and $g$, and also $\sigma_{A, B}^{\dagger}=\sigma_{B, A}$, where $\sigma_{A, B}: A \otimes B \rightarrow B \otimes A$ is the "swap" morphism, then $\mathcal{C}$ is said to be dagger-monoidal. Finally, if $\mathcal{C}$ admits a compact structure such that $\eta_{A}^{\dagger}=f_{A}$, then $\mathcal{C}$ is said to be dagger-compact.

It is not difficult to show that $*$-Alg is dagger-compact, where, if $\mathbf{M}$ and $\mathbf{N}$ are finite-dimensional $*$-algebras and $\phi: \mathbf{M} \rightarrow \mathbf{N}$ is a linear mapping, $\phi^{\dagger}$ is the hermitian adjoint of $\phi$ with respect to the natural trace inner products on $\mathbf{M}$ and $\mathbf{N}$. If $\left(A, \mathbf{M}_{A}\right)$ and $\left(B, \mathbf{M}_{B}\right)$ are involutive EJC-algebras with given unitary involutions $\Phi_{A}$ and $\Phi_{B}$, then for any intertwiner $\phi: \mathbf{M}_{A} \rightarrow \mathbf{M}_{B}, \phi^{\dagger}$ also intertwines $\Phi_{A}$ and $\Phi_{B}$. Hence, we have

Corollary 6.21. InvQM is dagger-compact.

\section{Conclusion}

We have constructed two categories of probabilistic models - the categories RSE and InvQM - that, in different ways, unify finite-dimensional real, complex and quaternionic quantum mechanics. In each case, there is a price to be paid for this unification. For RSE, this price is steep: RSE is a monoidal category, but one in which states (for instance) on complex systems don't count as physical processes. In particular, RSE is very far from being compact closed. ${ }^{28}$

In contrast, InvQM is clearly a well-behaved - indeed, dagger-compact - probabilistic theory, in which the states, as well as the effects, of real, complex, and quaternionic Euclidean Jordan algebras appear as morphisms. On the other hand, InvQM admits the transpose automorphism on the complex Hermitian Jordan algebra, and requires complex quantum systems to compose in a non-standard way. Nevertheless, by

\footnotetext{
${ }^{28}$ In [13], we erroneously claimed that the category URSE of universally reversible, but standardly embedded, EJC algebras, with relatively CJP mappings, is compact closed. That this is not the case is clear from Example 6.3.
} 
virtue of being dagger compact, InvQM continues to enjoy many of the informationprocessing properties of standard complex QM, e.g., the existence of conclusive teleportation and entanglement-swapping protocols [1]. Also, composites in InvQM satisfy the Cirel'son bound on correlations owing to the way that, by construction, these composites are embedded within a tensor product of complex matrix algebras.

All of this is in spite of the fact that composites in InvQM are not locally tomographic: the canonical composite $A \odot B$ is larger than the vector space tensor product $A \otimes B$. Local tomography is well known to separate complex QM from its real and quaternionic variants, so its failure in URUE and RSE is hardly surprising, but it is noteworthy that we are able to construct (non-locally tomographic) composites in URUE in all of the non-real cases, and certain composites involving quaternions even in RSE. Even more interesting is the fact that, for quaternionic systems $A$ and $B$, the information capacity - the number of sharply distinguishable states - of $A \odot B$ is larger than the product of the capacities of $A$ and $B$. A related point is that, for quaternionic quantum systems $A$ and $B$, the product of a pure state of $A$ and a pure state of $B$ will generally be a mixed state in $A \odot B$.

The category InvQM contains interesting compact closed subcategories. In particular, real and quaternionic quantum systems, taken together, form a (full) monoidal sub-category of InvQM closed under composition. We conjecture that this is exactly what one gets by applying Selinger's CPM construction [57] to Baez' (implicit) category of pairs $(\mathbf{H}, J), H$ a finite-dimensional Hilbert space and $J$ an anti-unitary with $J^{2}= \pm 1[7]$.

Another compact-closed subcateory of InvQM, which we might call InvCQM, consists of universally embedded complex quantum systems $C_{n}$. It is interesting to note that, in an hypothetical universe described by InvQM, the subcategory InvCQM acts as a kind of "ideal", in that if $A \in \mathbf{I n v Q M}$ and $B \in \mathbf{I n v C Q M}$, then $A \odot B \in \mathbf{I n v C Q M}$ as well. This is provocative, as it suggests that such a universe, initially consisting of many systems of all three types, might eventually evolve into one in which complex systems greatly predominate.

Although it is not compact closed, the category RSE of reversible, standardly embedded EJCs remains of interest. This is still a monoidal category, and contains, in addition to real and quaternionic quantum systems, orthodox complex quantum systems in their standard embedding (and composing in the normal way). Indeed, these form a monoidal subcategory, CQM, which, again, functions as an "ideal".

It is worth noting that the set of quaternionic quantum systems does not form a monoidal subcategory of either RSE or InvQM, as the composite of two quaternionic systems is real. Efforts to construct a free-standing quaternionic quantum theory have had to contend with the absence of a suitable quaternionic composite of quaternionic systems. For instance, as pointed out by Araki [5], the obvious candidate for the composite of $A=M_{m}(\mathbb{H})_{s a}$ and $B=M_{n}(\mathbb{H})_{s a}, M_{m n}(\mathbb{H})_{s a}$, does not have a large enough dimension to accommodate the real tensor product $A \otimes B$, causing difficulty 
for the representation of product effects. ${ }^{29}$ In our approach, the issue simply doesn't arise. It seems that "quaternionic quantum mechanics" is best seen as an inextricable part of a larger theory. Essentially the same point has also been made by Baez [7].

The category InvQM is somewhat mysterious. It encompasses real and quaternionic QM in a completely natural way; however, while it also contains complex quantum systems, these compose in an exotic manner: as pointed out above, the composite of two complex quantum systems in InvQM comes with an extra classical bit - equivalently, $\{0,1\}$-valued superselection rule. This functions to make the transpose automorphism of $M_{n}(\mathbb{C})_{s a}$ count as a morphism. The extra classical bit is flipped by the Jordan transpose (swap of $C^{*}$ summands) on either factor of such a composite, but unaffected if both parties implement the Jordan transpose (which does, of course, effect a Jordan transpose on the composite). The precise physical significance of this is a subject for further study.

As Example 6.2 shows, there is no way to enlarge InvQM so as to include higher spin factors, without either sacrificing compact closure (and even rendering the set $\mathcal{C}(A, I)$, which might naturally be thought to represent states, trivial) or venturing outside the ambient category of EJC-algebras, to make use of morphisms that are not (relatively) completely Jordan-preserving maps. Example 6.3 shows, more strikingly, that there is no way to construct a category of the form $\mathbf{C J P}_{\mathcal{C}}$ that contains standardly embedded complex quantum systems and real systems, without, again, sacrificing compact closure (indeed, the representation of states by morphisms).

\section{References}

[1] S. Abramsky and B. Coecke, Categorical quantum mechanics, in D. Gabbay, K. Engesser and D. Lehman, Handbook of Quantum Logic and Quantum Structures vol II, Elsevier, 2008; doi:10.1016/B978-0-444-52869.5001-4 arXiv : quant-ph/0402130)

[2] E. M. Alfsen and F. W. Shultz, State spaces of Jordan algebras, Acta Mathematica 140 (1978) 155-190 doi:10.1007/BF02392307

[3] E. M. Alfsen and F. W. Shultz, State Spaces of Operator Algebras: Basic Theory, Orientations and $C^{*}$-Products, Birkhauser, 2001 doi:10.1007/978-1-4612-1047-2

[4] E. Alfsen and F. Shultz, Geometry of state spaces of operator algebras, Birkhäuser, 2003 doi:10.1007/978-1-4612-0019-2

[5] H. Araki, On a characterization of the state space of quantum mechanics, Comm. Math. Phys. 75, 1980, 1-24 doi:10.1007/BF01962588

\footnotetext{
${ }^{29}$ Attempts to interpret the quaternionic "Hilbert space" $\mathbb{H}^{m n}$ as a tensor product of $\mathbb{H}^{m}$ and $\mathbb{H}^{n}$ raise at least the possibility of signaling via the noncommutativity of scalar multiplication. This noncommutativity underlies the the argument in [50] that stronger-than-quantum correlations are achievable in such a model.
} 
[6] C. Aliprantis and D. Toukey, Cones and Duality, Springer, 2007 doi: $10.1090 / \mathrm{gsm} / 084$

[7] J. Baez, Division algebras and quantum theory, Foundations of Physics 42 819-855 (2012) doi:10.1007/s10701-011-9566-Z arXiv:1101.5690

[8] J. Baez, Quantum quandaries: a category-theoretic perspective in D. Rickles, S. French and J. Saatsi, Eds., The Structural Foundations of Quantum Gravity, Oxford, 2006, 240-265 (quant-ph/0404040

[9] H. Barnum, J. Barrett, M. Leifer and A. Wilce, Cloning and broadcasting in generic probabilistic theories, preprint, 2006 arxiv:quant-ph/0611295

[10] H. Barnum, J. Barrett, M. Leifer and A. Wilce, Teleportation in general probabilistic theories, in S. Abramsky and M. Mislove, Eds., Mathematical Foundations of Information Flow (Proceedings of the Clifford Lectures 2008), Proceeding of Symposia in Applied Mathematics 71, American Mathematical Society, Providence, 2012, 25-48 doi:http://dx.doi.org/10.1090/psapm/071/600 arXiv:0805.3553

[11] H. Barnum, R. Duncan and A. Wilce, Symmetry, compact closure and dagger compactness for categories of convex operational models, Journal of Philosophical Logic 42 (2013) 501-523 doi:http://dx.doi.org/10.1007/s10992-013-9280-8 arXiv: 1004.2920

[12] H. Barnum, C. Gaebler and A. Wilce, Ensemble steering, weak self-duality and the structure of probabilistic theories, Foundations of Physics 43 1411-1437 (2013) doi:10.1007/s10701-013-9752-2; arxiv:0912.5532

[13] H. Barnum, M. Graydon and A. Wilce, Some Nearly Quantum Theories, EPTCS 195 (2015), 59-70 doi:10.4204/EPTCS.195.5 arXiv:1507.06278

[14] H. Barnum and J. Hilgert, Spectral properties of convex bodies, J. Lie Theory 30, 315-344 (2020). (ArXiv version: Strongly symmetric spectral convex bodies are Jordan algebra state spaces arxiv: 1904.03753 (2019))

[15] H. Barnum, M. Mueller and C. Ududec, Higher-order interference and singlesystem postulates characterizing quantum theory, New J. Physics 16 (2014) doi:10.1088/1367-2630/16/12/123029 arXiv:1403.4147

[16] H. Barnum and A. Wilce, Information processing in convex operational theories, Electronic Notes in Theoretical Computer Science 270 (2011) 3-15 doi:10.1016/j.entcs.2011.01.002 arXiv:0908.2352

[17] H. Barnum and A. Wilce, Local tomography and the Jordan structure of quantum theory, Found. Phys. 44 (2014), 192-212 doi:10.1007/s10701-014-9777-1 arXiv: 1202.4513

[18] H. Barnum and A. Wilce, Post-classical probability theory, in G. Chiribella and R. Spekkens, eds., Quantum Theory: Informational Foundations and Foils, Springer, 2017 doi:10.1007/978-94-017-7303-4_11; arXiv : 1205.3833 
[19] J. Barrett, Information processing in generalized probabilistic theories, Physical Review A 75 (2005) doi:10.1103/PhysRevA.75.032304 arXiv:quant-ph/0508211

[20] L. J. Bunce and J. D. Maitland-Wright, Introduction to the $K$ theory of Jordan $C^{*}$ algebras, Quart. J. Math. 40 (1989) 377-398 doi:http://dx.doi.org/10.1093/qmath/40.4.377

[21] G. Chiribella, M. D'Ariano and P. Perinotti, Informational derivation of quantum theory, Physical Review A 84 (2011), 012311 doi:10.1103/PhysRevA.84.012311 arXiv:1011.6451

[22] M. L. Curtis, Matrix Groups, Springer, 1984 doi:http://dx.doi.org/10.1007/978-14612-5286-3

[23] B. Dakic and C. Brukner, Quantum theory and beyond: is entanglement special? in H. Halvorson, ed., Deep Beauty, Princeton, 2011 doi:10.1017/CBO9780511976971 arXiv:0911.0695

[24] E. B. Davies and J. Lewis, An operational approach to quantum probability, Comm. Math. Phys. 17 (1970) 239-260 doi:10.1007/BF01647093

[25] C. M. Edwards, The operational approach to algebraic quantum theory I, Comm. Math. Phys. 16 (1970), 207-230 doi:10.1007/bf01646788

[26] J. Faraut and A. Korányi, Analysis on Symmetric Cones, Oxford, 1994

[27] M. A. Graydon, Conical Designs and Categorical Jordan Algebraic Post-Quantum Theories, Ph. D. Dissertation, University of Waterloo, 2017 arXiv:1703.06800 [quant-ph]

[28] M. A. Graydon, Quaternions and Quantum Theory, Master's Thesis, University of Waterloo, 2011

[29] H. Hanche-Olsen, JB algebras with tensor products are $C^{*}$ algebras, in H. Araki et al. (eds.), Operator Algebras and their Connections with Topology and Ergodic Theory, Lecture Notes in Mathematics 1132 (1985), 223-229 doi:10.1007/BFb0074886

[30] H. Hanche-Olsen, On the structure and tensor products of JC-algebras, Can. J. Math. 35 (1983), 1059-1074 doi:http://dx.doi.org/10.4153/CJM-1983-059-8

[31] H. Hanche-Olsen and E. Størmer, Jordan Operator Algebras, Pitman, 1984 (Out of print; available at https://folk.ntnu.no/hanche/joa/)

[32] L. Hardy, Quantum theory from five reasonable axioms, arXiv:quant-ph/0101012, 2001

[33] L. Hardy and W. Wootters","Limited holism and real-vector-space quantum theory, Found. Phys. 42 (2012) 454-473 doi:10.1007/s10701-011-9616-6 arXiv: 1005.4870

[34] J. Hilgert, K. H. Hoffman and J. D. Lawson, Lie Groups, Convex Cones, and Semigroups, Oxford, 1989 
[35] A. Holevo, Probabilistic and Statistical Aspects of Quantum Mechanics, Springer Basel, 2011 doi:10.1007/978-88-7642-378-9

[36] F. B. Jamjoom, On the tensor products of JC-algebras, Quart. J. Math. Oxford 45 (1994) 77-90 doi:http://dx.doi.org/10.1093/qmath/45.1.77

[37] F. B. Jamjoom, On the tensor products of Simple JC algebras, Mich. Math. J. 41 (1994), 289-295 doi:http://dx.doi.org/10.1307/mmj/1029004996

[38] F. B. Jamjoom, On the tensor products of JW-algebras, Can. J. Math. 47 (1995) 786-8000 doi:http://dx.doi.org/10.4153/CJM-1995-040-1

[39] P. Janotta and R. Lal, Generalized probabilistic theories without the norestriction hypothesis, Phys. Rev. A. 87 (2013) doi:10.1103/PhysRevA.87.052131 arXiv:1302.2632

[40] Über die Multiplikation quantenmechanischer Größen, Zeit. Phys. 80 (1933), 285291. (Also see [41, 42].)

[41] P. Jordan, Über eine Klasse nichtassociativer hyperkomplexer Algebren, Nachrichten von der Gesellschaft der Wissenschaften zu Göttingen, MathematischPhysikalische Klasse 33 (1932) 569-575,

[42] P. Jordan, Über Verallgemeinerungsmöglichkeiten des Formalismus Quantenmechanik, Nachrichten von der Gesellschaft der Wissenschaften zu Göttingen, Mathematisch-Physikalische Klasse 39 (1933), 209-217

[43] P. Jordan, J. von Neumann and E. Wigner, On an algebraic generalization of the quantum mechanical formalism, Annals of Math. 35 (1934), 29-64 doi:10.2307/1968117

[44] M. Koecher, The Minnesota Notes on Jordan Algebras and their Applications, Ed. A. Krieg and S. Walcher, Springer Lecture Notes in Mathematics 1710, Springer, 1999 doi:10.1007/BFb0096285

[45] Die Geodätischen von Positivitätsbereichen, Math. Ann. 135 (1958) 192-202. doi:http://dx.doi.org/10.1007/BF01351796

[46] G. Ludwig, Deutung des Begriffs, "physikalische Theorie" und axiomatische Grundlegung der Hilbertsraumstruktur der Quantenmechanik durch Hauptsätze des Messens Springer Lecture Notes in Physics 41970

[47] G. Ludwig, An axiomatic basis for quantum mechanics. Volume 1: Derivation of Hilbert Space Structure, Springer, 1985

[48] H. Neumann and A. Hartkämper, Eds., Foundations of Quantum Mechanics and Ordered Linear Spaces, Springer, Lecture Notes in Physics 29, 1974 doi:10.1007/3540-06725-6

[49] H. Neumann, The structure of ordered Banach spaces in axiomatic quantum mechanics, in H. Neumann and A. Hartkämper, Eds., Foundations of Quantum Mechanics and Ordered Linear Spaces, Springer, Lecture Notes in Physics 29 (1974) 116-121 doi:http://dx.doi.org/10.1007/3-540-06725-6_13 
[50] M. McKague, Quaternionic quantum mechanics allows non-local boxes, Preprint, arXiv:0911.1761, 2009

[51] Ll. Masanes and M. Müller, A derivation of quantum theory from physical requirements, New J. Phys. 13 (2011) doi:10.1088/1367-2630/13/6/063001 arXiv:1004.1483

[52] M. Mueller and Ll. Masanes, Information-theoretic postulates for quantum mechanics, in G. Chiribella and R. Spekkens, eds. Quantum Theory: Informational Foundations and Foils, Springer, 2016 doi:10.1007/978-94-017-7303-4_5; arXiv: 1203.451

[53] M. Müller and C. Ududec, The structure of reversible computation determines the self-duality of quantum theory, Phys. Rev. Lett. 108 (2012), 130401 doi:10.1103/PhysRevLett.108.130401 arXiv:1110.3516

[54] A. G. Robertson, Automorphisms of spin factors and the decomposition of positive maps, Quart. J. Math. Oxford Ser. 34 (1983) 87-96 doi:http://dx.doi.org/10.1093/qmath/34.1.87

[55] I. Satake, Linear embeddings of self-dual homogeneous cones, Nagoya Math. J. 46 (1972) 121-145 doi:10.1017/S0027763000014811

[56] I. Satake, Algebraic structures of symmetric domains, Publications of the Mathematical Society of Japan 14, Iwanami Shoten and Princeton University Press, 1980

[57] P. Selinger, Dagger compact categories and completely positive maps (extended abstract), in Proceedings of the 3d International Workshop on Quantum Programming Languages (QPL 2005), Electronic Notes in Theoretical Computer Science 170 (2007) 139-163 doi:10.1016/j.entcs.2006.12.018

[58] A. M. Sinclair, Jordan homomorphisms and derivations of semi-simple Banach algebras, Proc. Amer. Math. Soc. 24 (1970) 209-214 doi:10.2307/2036730

[59] E. Størmer, Jordan algebras of type I, Acta Math. 115 (1966), 165-184 doi:10.1007/BF02392206

[60] D. Topping, Jordan Algebras of Self-Adjoint Operators, Am. Math. Soc. Memoirs 53, Providence, 1965 doi:10.1090/memo/0053

[61] H. Upmeier, Derivations ofJordan $C^{*}$-algebras, Math. Scand. 46 (1980) 251-264 doi:10.7146/math.scand.a-11867

[62] E. Vinberg, Homogeneous cones, Dokl. Acad. Nauk. SSSR 141 (1960) 270-3. English translation: E. Vinberg (1961): Soviet Math. Dokl. 2, pp. 1416-1619.

[63] A. Wilce, Four and a half axioms for finite-dimensional quantum theory in Y. Ben-Menahem and M. Hemmo (eds.), Probability in Physics, Springer, 2012 doi:10.1007/978-3-642-21329-8_17; arXiv:0912.5530 
[64] A. Wilce, Symmetry and self-duality in categories of probabilistic models, in B. Jacobs and P. Selinger, Eds., Proceedings of QPL 2011, Electronic Proceedings in Theoretical Computer Science 95 (2012) 289-293 doi:http://dx.doi.org/10.4204/EPTCS.95.19 arXiv:1210.0622

[65] A. Wilce, A royal road to quantum theory (or thereabouts), Entropy 20 (2018), 227-253 doi:10.3390/e20040227 arXiv:1606.09306

[66] A. Wilce, Conjugates, filters and quantum mechanics, Quantum 3 (2019) 158-191 doi:http://dx.doi.org/10.22331/q-2019-07-08-158 arXiv:1206. 2897

\section{A Direct Sums of EJAs}

The first part of this Appendix collects some basic facts about direct sums of EJAs that are used in the body of the paper. The second part contains a proof that $C^{*}(A \oplus B)=$ $C^{*}(A) \oplus C^{*}(B)$, and that the direct sum of universally reversible EJAs is again UR.

Direct Summands and Central Projections The direct sum of EJAs $A$ and $B$ is $A \oplus B:=A \times B$, equipped with the slotwise operations, so that the canonical projections $\pi_{1}: A \times B \rightarrow A$ and $\pi_{2}: A \times B \rightarrow B$ are unital Jordan homomorphisms. Identifying $A$ and $B$ with $A \times\{0\}$ and $\{0\} \times B$, respectively, we write $a+b$ for $(a, 0)+(0, b)$. Note that $A$ and $B$ are then ideals in $A \oplus B$, and that $B=A^{\perp}:=\{z \in A \oplus B \mid\langle a, z\rangle=0 \forall a \in A\}$. Conversely, we will show that if $E$ is an EJA and $A$ is an ideal in $A$, then $A^{\perp}$ is also an ideal, and $a \bullet b=0$ for all $a \in A, b \in A^{\perp}$; hence, $E \simeq A \oplus A^{\perp}$.

Suppose $E$ is an EJA and $A \leq E$ is an ideal: let $B=A^{\perp}$. Then for all $z \in E, a \in A$ and $b \in B$,

$$
\langle a, z \bullet b\rangle=\langle a \bullet z, b\rangle=0
$$

since $a \bullet z \in A$. Thus, $B$ is also an ideal, and $E=A \oplus B$ as a vector space. Finally, if $a \in A$ and $b \in B$, then $a \bullet b \in A \cap B=\{0\}$. Hence, if $a, x \in A$ and $b, y \in B$ then $(a+b) \bullet(x+y)=a \bullet x+b \bullet y$, i.e., in the representation of $A \oplus B$ as $A \times B$, operations are slotwise. Note that $u=p_{A}+p_{B}$ for a unique $p_{A} \in A$ and $p_{B} \in B$. For $a \in A$ we have $a \bullet p_{B}=0$, so $a=a \bullet u=a \bullet p_{A}$, whence $p_{A}=p_{A}^{2}$ and $A=A \bullet p_{A}$. In other words, we have

Lemma A.1. Let $A$ be an ideal in an EJA E. Then there exists a projection $p \in E$ such that $p \bullet a=a$ for every $a \in A$. Thus, $A=p \bullet A$, and $E=p \bullet A \oplus p^{\prime} \bullet A$, where $p^{\prime}=1-p$.

The center of an EJA $E$ is the set of elements operator-commuting with all other elements. Denote this by $C(E)$. If $E=A \oplus B$, and $p$ is the unit of $A$, so that $A=p \bullet A$, then it's easy to check that $p \in C(E)$. Conversely, if $p$ is a central projection, then $p \bullet A$ is an ideal, with unit element $p$. If $p$ is a minimal central projection, then $p \bullet A$ is a minimal direct summand of $E$. If $E$ is simple, then its only central projections are 0 and 1 , and conversely. 
One can show that for every projection $p$ in an EJA $E$, there exists a unique minimal projection $c(p) \in C(E)$, the central cover of $p$, such that $p \leq c(p)$. Then $A:=c(p) E$ is an ideal of $E$, in which $c(p)$ is the unit. If $A$ is a minimal ideal, then elements of $A$ are exactly those with central cover $c(p)$ (see 2.37 and 2.39 in [4].) More generally, two elements of $E$ having the same central cover have nonzero components in exactly the same ideals of $E$.

Recall that a symmetry of $A$ is an element $s \in A$ with $s^{2}=u$. Projections $e, f$ in $A$ are exchanged by a symmetry $s$ iff $U_{s}(e)=f$. If there exists a sequence of symmetries $s_{1}, \ldots, s_{n}$ with $U_{s_{n}} \circ \cdots \circ U_{s_{1}}(e)=f$, then $e$ and $f$ are equivalent.

Lemma A.2 ([4], Lemma 3.9). Equivalent projections have the same central cover.

The universal $C^{*}$-algebra of a direct sum Recall that a sequence of vector spaces and linear maps, or of Jordan algebras and Jordan homomorphisms, or of $C^{*}$ algebras and $*$-homomorphisms

$$
A \stackrel{\alpha}{\longrightarrow} B \stackrel{\beta}{\longrightarrow} C
$$

is said to be exact at $B$ iff the image of $\alpha$ is the kernel of $\beta$. A short exact sequence is one of the form

$$
0 \longrightarrow A \stackrel{\alpha}{\longrightarrow} B \stackrel{\beta}{\longrightarrow} C \longrightarrow 0
$$

that is exact at $A, B$ and $C$ (with the maps on the ends being the only possible ones). This means that $\alpha$ is injective (its kernel is 0 ), while $\beta$ is surjective (its image is the kernel of the zero map, i.e., all of $C$ ).

Let EJA and $C^{*}$ be the categories of EJAs and Jordan homomorphisms, and of $C^{*}$-algebras and $*$-homomorphisms, respectively.

Theorem A.3 ([30]). $A \mapsto C^{*}(A)$ is an exact functor from EJA to $C^{*}$. In other words, if $A \stackrel{\alpha}{\longrightarrow} B \stackrel{\beta}{\longrightarrow} C$ is an exact sequence in $\mathbf{E J A}$, then $C^{*}(A) \stackrel{C^{*}(\alpha)}{\longrightarrow} B \stackrel{C^{*}(\beta)}{\longrightarrow} C^{*}(C)$ is an exact sequence in $C^{*}$.

We are going to use this to show that $C^{*}(A \oplus B)=C^{*}(A) \oplus C^{*}(B)$. We need some preliminaries. The following is standard:

Lemma A.4. Let

$$
0 \longrightarrow A \stackrel{\alpha}{\longrightarrow} C \stackrel{\beta}{\longrightarrow} B \longrightarrow 0
$$

be a short exact sequence of vector spaces. Then the following are equivalent:

(a) There is an isomorphism $\phi: A \oplus B \simeq C$ such that $\alpha$ and $\beta$ are respectively the canonical injection and surjection given by

$$
\alpha(a)=\phi(a, 0) \text { and } \beta(\phi(a, b))=b
$$

(b) The sequence is split at $B$ : there exists a linear mapping $\gamma: B \rightarrow C$ such that $\beta \circ \gamma=i d_{B}$. 
The idea is that, given $\phi$, we can define $\gamma$ by $\gamma(b)=\phi(0, b)$ and, given $\gamma$, we can define $\phi$ by $\phi(a, b)=\alpha(a)+\gamma(b)$.

If $A, B$ and $C$ are Jordan algebras or $C^{*}$ algebras, the implication from (a) to (b) is obviously valid, but the converse requires additional assumptions.

Lemma A.5. Let

$$
0 \longrightarrow A \stackrel{\alpha}{\longrightarrow} C \stackrel{\beta}{\longrightarrow} B \longrightarrow 0 \text {. }
$$

be a short exact sequence of *-algebras and *-homomorphisms, which is split by a*homomorphism $\gamma: B \rightarrow C$ with $\beta \circ \gamma=i d_{B}$. Let $\phi: A \oplus B \rightarrow C$ be as defined above, i.e, $\phi(a, b)=\alpha(a)+\gamma(b)$ for $a \in A, b \in B$. Then the following are equivalent:

(a) $\gamma(B)$ is a (2-sided) *-ideal in $C$;

(b) $\phi$ is multiplicative, and thus a *-isomorphism;

(c) There exists a *-homomorphism $\delta: C \rightarrow A$ with

$$
0 \longleftarrow A \stackrel{\delta}{\longleftarrow} C \stackrel{\gamma}{\longleftarrow} B \longleftarrow 0
$$

exact.

Proof: $(\mathrm{a}) \Rightarrow(\mathrm{b})$. It is easy to see that a $C^{*}$-algebra $C$ is the direct sum of two $*$-ideals $A, B \leq C$ iff $A \oplus B=C$ and $A \cap C=\{0\}$, i.e., iff $C$ is the vector-space direct sum of $A$ and $B$. We already know that $\alpha(A)+\beta(B)=C$ (since $\phi$ is a linear isomorphism). It therefore suffices to show that $\alpha(A)$ and $\gamma(B)$ are $*$-ideals with zero intersection. We are assuming that $\gamma(B)$ is a $*$-ideal. As it's the kernel of a $*$-homomorphism, $\alpha(A)$ is automatically a $*$-ideal. To see that $\alpha(A) \cap \gamma(C)=\{0\}$, let $c \in C$ with $c=\alpha(a)=\gamma(b)$ for some $a \in A$ and $b \in B$. Then we have

$$
b=\beta(\gamma(b))=\beta(\alpha(a))=0
$$

whence, $c=\gamma(0)=0$.

(b) $\Rightarrow$ (c). If $\phi$ is a $*$-isomorphism, then let $\delta=\pi_{A} \circ \phi^{-1}$ where $\pi_{A}: A \oplus B \rightarrow A$ is the projection $\pi_{A}(a, b)=a$. Note that $\delta$ is the composition of two $*$-homomorphisms, and thus, a $*$-homomorphism. To verify exactness, note that as $\phi(a, b)=\alpha(a)+\gamma(b)$, we have $\phi(0, b)=\gamma(b)$, whence, $\phi^{-1}(\gamma(b))=(0, b)$. Thus, $\delta(\gamma(b))=\pi_{A}(0, b)=0$.

$(\mathrm{c}) \Rightarrow(\mathrm{a})$. By exactness, $\gamma(C)$ is the kernel of the $*$-homomorphism $\delta$, and hence, a $*$-ideal.

Now let $E=A \oplus B$. Then we have a short exact sequence

$$
0 \longrightarrow A \stackrel{j}{\longrightarrow} A \oplus B \stackrel{p}{\longrightarrow} B \longrightarrow 0 .
$$

where $j(a)=(a, 0)$ and $p(a, b)=b$. This is split by the homomorphism $k: A \rightarrow A \oplus B$ given by $k(b)=(0, b)$. Hanche-Olsen's exactness theorem, that is Theorem A.3, gives us a short exact sequence

$$
0 \longrightarrow C^{*}(A) \stackrel{C^{*}(j)}{\longrightarrow} C^{*}(A \oplus B) \stackrel{C^{*}(p)}{\longrightarrow} C^{*}(B) \longrightarrow 0
$$


By functoriality, $C^{*}(p) \circ C^{*}(j)=\mathrm{id}_{C^{*}(B)}$, so this is again split. Thus, regarded as a vector space, $C^{*}(A \oplus B)$ is canonically isomorphic to $C^{*}(A) \oplus C^{*}(B)$. On the other hand, we also have an exact sequence

$$
0 \longleftarrow A \stackrel{q}{\longleftarrow} A \oplus B \stackrel{k}{\longleftarrow} B \longleftarrow 0
$$

where $q(a, b)=a$. By the same argument, then, we have a short exact sequence

$$
0 \longleftarrow C^{*}(A) \stackrel{C^{*}(q)}{\longleftarrow} C^{*}(A \oplus B) \stackrel{C^{*}(k)}{\longleftarrow} C^{*}(B) \longleftarrow 0 .
$$

Applying the preceding Lemma, we have

Proposition A.6. If $A$ and $B$ are EJAs, then

$$
C^{*}(A \oplus B) \simeq C^{*}(A) \oplus C^{*}(B)
$$

Notice that if $\Phi_{A}$ and $\Phi_{B}$ are, respectively, the canonical involutions on $C^{*}(A)$ and $C^{*}(B)$ fixing points of $A$ and $B$, then $\Phi_{A} \oplus \Phi_{B}$ is a $*$-involution on $C^{*}(A) \oplus C^{*}(B)$ fixing points of $A \oplus B$. But there is only one such $*$-involution on $C^{*}(A \oplus B)$, the canonical one. In other words, in identifying $C^{*}(A \oplus B)$ with $C^{*}(A) \oplus C^{*}(B)$, we also identify $\Phi_{A \oplus B}$ with $\Phi_{A} \oplus \Phi_{B}$.

Recalling now the fact ([30], Lemma 4.2) that an EJA $A$ is universally reversible (UR) iff $A$ coincides with the set of self-adjoint fixed points in $C^{*}(A)$ of the canonical *-involution $\Phi_{A}$, we have the following.

Corollary A.7. $A \oplus B$ is UR iff $A$ and $B$ are UR.

Proof: Let $\Phi=\Phi_{A} \oplus \Phi_{B}$ be the canonical involution on $C^{*}(A \oplus B)=C^{*}(A) \oplus C^{*}(B)$. For $(a, b) \in C^{*}(A) \oplus C^{*}(B)$, we have $\Phi(a, b)=\left(\Phi_{A}(a), \Phi_{B}(b)\right)=(a, b)$ iff $\Phi_{A}(a)=a$ and $\Phi_{B}(b)=b$. Since $A$ and $B$ are UR, this holds iff $a \in A$ and $b \in B$, i.e., iff $(a, b) \in A \oplus B$. Thus, $A \oplus B$ is exactly the set of fixed-points of $\Phi$, and so, is UR.

Conversely, let $A \oplus B$ be UR. Suppose for a contradiction that one of $A$ or $B$, say $B$, is not UR: then there exists $b \in C^{*}(B)$ such that $\Phi_{B}(b)=b$ but $b \notin B$. Let $a$ be in $A$. Then $\Phi_{A \oplus B}((a, b)) \equiv\left(\Phi_{A}(a), \Phi_{B}(b)\right)=(a, b)$, whence by the fact that $A \oplus B$ is UR, $(a, b) \in A \oplus B$, but since $b \notin B$ this is in contradiction with the definition of $A \oplus B$ as $A \times B$ equipped with a product.

(One can also easily prove the above Corollary directly from the definition of universal reversibility, without using the canonical involutions.)

\section{B The Quabit}

In this appendix, we show that the canonical tensor product $Q_{2} \odot Q_{2}$ of two quabits in their standard representation is $R_{16}$, the self-adjoint part of the real matrix algebra $M_{16}(\mathbb{R})$.

Accepted in 〈 Xuantum 2020-10-07, click title to verify. Published under CC-BY 4.0. 
The symplectic representation A quaternion $q=a+b i+c j+d k$ can alternatively be expressed in the form $(a+b i)+(c+d i) j$, i.e., $z+w j$ where $z, w \in \mathbb{C}$, and also has a standard representation as a $2 \times 2$ complex matrix, namely

$$
[q]:=\left[\begin{array}{cc}
z & w \\
-\bar{w} & \bar{z}
\end{array}\right] .
$$

Treating $\mathbb{H}$ as a $*$-algebra over $\mathbb{C}$, the mapping $q \mapsto[q]$ is a $*$-homomorphism from $\mathbb{H}$ into $M_{2}(\mathbb{C})$. This yields a natural representation - that is, $*$-homomorphism $\pi_{o}: M_{n}(\mathbb{H}) \rightarrow M_{n}\left(M_{2}(\mathbb{C})\right) \simeq M_{2 n}(\mathbb{C})$, given by

$$
\pi_{o}(a)_{p, q}=\left[a_{p, q}\right]
$$

for $a \in M_{n}(\mathbb{H})$ and $p, q=1, \ldots, n$. An equivalent, but for our purposes, more useful, representation is given by

$$
\pi(a)_{p, q}=F \pi_{o}(a)_{p, q} F
$$

where

$$
F=\left(\begin{array}{llll}
1 & 0 & 0 & 0 \\
0 & 0 & 1 & 0 \\
0 & 1 & 0 & 0 \\
0 & 0 & 0 & 1
\end{array}\right) .
$$

This is called the symplectic representation of $M_{n}(\mathbb{H})$.

If we express each entry of $a$ in the form $a_{p, q}=z_{p, q}+w_{p, q} j$, then $a=\Gamma_{1}+\Gamma_{2} j$ where $\left(\Gamma_{1}\right)_{p, q}=z_{p, q}$ and $\left(\Gamma_{2}\right)_{p, q}=w_{p, q}$. Computing, one finds that

$$
\pi(a)=\left[\begin{array}{cc}
\Gamma_{1} & \Gamma_{2} \\
-\bar{\Gamma}_{2} & \bar{\Gamma}_{1}
\end{array}\right] .
$$

Notice that $\pi(a)$ is self-adjoint iff $\Gamma_{1}$ is self-adjoint and $\Gamma_{2}$ is anti-symmetric, and that this is the case iff $a$ is self-adjoint in $M_{2}(\mathbb{H})$.

From now on, we identify $Q_{2}$ with $\pi\left(M_{2}(\mathbb{H})_{s a}\right) \leq M_{4}(\mathbb{C})_{s a}$. Regarded as an embedded EJA in this way, the canonical tensor product $Q_{2} \odot Q_{2}$ is defined to be the Jordan subalgebra of the self-adjoint part of $M_{4}(\mathbb{C}) \otimes M_{4}(\mathbb{C})=M_{16}(\mathbb{C})$ generated by $Q_{2} \otimes Q_{2}$. Our main goal in this Appendix is to prove

Proposition B.1. $C^{*}\left(Q_{2} \odot Q_{2}\right) \simeq M_{16}(\mathbb{C})$.

Since $Q_{2} \odot Q_{2}$ is UR, this will follow from ([30], Theorem 4.4) if we can show that (i) $Q_{2} \odot Q_{2}$ generates $M_{16}(\mathbb{C})$ as a $*$-algebra, and (ii) there is a $*$-involution on $M_{16}(\mathbb{C})$ fixing elements of $Q_{2} \odot Q_{2}$ pointwise.

Quaternionic Pauli Matrices In order to show that $Q_{2} \odot Q_{2}$ generates $M_{16}(\mathbb{C})$, we begin by writing down some useful elements of $Q_{2}$. The analogues of the Pauli matrices 
$\sigma_{x}, \sigma_{y}, \sigma_{z} \in M_{2}(\mathbb{C})$ are the following quaternionic Pauli matrices:

$$
\begin{array}{ccc}
\sigma_{0}=\left[\begin{array}{ll}
1 & 0 \\
0 & 1
\end{array}\right] & \sigma_{1}=\left[\begin{array}{rr}
1 & 0 \\
0 & -1
\end{array}\right] & \sigma_{2}=\left[\begin{array}{ll}
0 & 1 \\
1 & 0
\end{array}\right] \\
\sigma_{3}=\left[\begin{array}{rr}
0 & -i \\
i & 0
\end{array}\right] & \sigma_{4}=\left[\begin{array}{rr}
0 & -j \\
j & 0
\end{array}\right] & \sigma_{5}=\left[\begin{array}{rr}
0 & -k \\
k & 0
\end{array}\right]
\end{array}
$$

Evidently, $\sigma_{1}, \sigma_{2}$ and $\sigma_{3}$ are the standard Pauli matrices $\sigma_{z}, \sigma_{x}$ and $\sigma_{y}$, respectively. Note that these are all traceless and self-adjoint, and satisfy $\sigma_{a} \bullet \sigma_{b}=\delta_{a, b} \sigma_{0}$ where $a, b \in\{1, \ldots, 5\}$ - that is, $\sigma_{a}$ and $\sigma_{b}$ anti-commute if $a \neq b$, and $\sigma_{a}$ squares to the identity. Applying the representation $\pi$ gives us six elements of $Q_{2}, s_{0}=\pi\left(\sigma_{0}\right)$, $s_{1}=\pi\left(\sigma_{1}\right), \ldots, s_{5}=\pi\left(\sigma_{5}\right)$. Direct computation reveals that

$$
\begin{aligned}
& s_{0}=\left[\begin{array}{cc}
\mathbf{1} & 0 \\
0 & \mathbf{1}
\end{array}\right]=\sigma_{0} \otimes \sigma_{0} \quad s_{1}=\left[\begin{array}{cc}
\sigma_{z} & 0 \\
0 & \sigma_{z}
\end{array}\right]=\sigma_{0} \otimes \sigma_{z} \quad s_{2}=\left[\begin{array}{cc}
\sigma_{x} & 0 \\
0 & \sigma_{x}
\end{array}\right]=\sigma_{0} \otimes \sigma_{x} \\
& s_{3}=\left[\begin{array}{cc}
\sigma_{y} & 0 \\
0 & -\sigma_{y}
\end{array}\right]=\sigma_{z} \otimes \sigma_{y} \quad s_{4}=\left[\begin{array}{cr}
0 & -i \sigma_{y} \\
i \sigma_{y} & 0
\end{array}\right]=\sigma_{y} \otimes \sigma_{y} \quad s_{5}=\left[\begin{array}{cc}
0 & \sigma_{y} \\
\sigma_{y} & 0
\end{array}\right]=\sigma_{x} \otimes \sigma_{x}
\end{aligned}
$$

These again obey the Pauli-like identities mentioned above. Using these, we can compute (associative) products of these matrices, e.g.,

$$
s_{3} s_{4}=\left(\sigma_{z} \otimes \sigma_{y}\right)\left(\sigma_{y} \otimes \sigma_{y}\right)=\sigma_{z} \sigma_{y} \otimes \sigma_{y} \sigma_{y}=-i \sigma_{x} \otimes \sigma_{0} .
$$

Lemma B.2. $Q_{2} \otimes Q_{2}$ generates $M_{16}(\mathbb{C})$ as a *-algebra.

Proof: Begin by noting that the elements

$$
\sigma_{a} \otimes \sigma_{b} \otimes \sigma_{c} \otimes \sigma_{c}
$$

where $a, b, c, d \in\{0, x, y, z\}$, are a basis for $M_{16}(\mathbb{C})$. For each $a \in\{0, x, y, z\}$, let

$$
\begin{aligned}
& x_{1}(a)=\sigma_{a} \otimes \sigma_{0} \otimes \sigma_{0} \otimes \sigma_{0} \\
& x_{2}(a)=\sigma_{0} \otimes \sigma_{a} \otimes \sigma_{0} \otimes \sigma_{0} \\
& x_{3}(a)=\sigma_{0} \otimes \sigma_{0} \otimes \sigma_{a} \otimes \sigma_{0} \\
& x_{4}(a)=\sigma_{0} \otimes \sigma_{0} \otimes \sigma_{0} \otimes \sigma_{a}
\end{aligned}
$$

Then $x_{1}(a) x_{2}(b) x_{3}(c) x_{4}(d)=\sigma_{a} \otimes \sigma_{b} \otimes \sigma_{c} \otimes \sigma_{d}$. These last form a basis for $M_{16}(\mathbb{C})$, so it will suffice to show that, for $a \in\{x, y, z\}$, the elements $x_{p}(a)$ can be manufactured from elements of $Q_{2} \otimes Q_{2}$ by forming (associative) products.

For a start, notice that

$$
s_{1} \otimes s_{0}=\sigma_{0} \otimes \sigma_{z} \otimes \sigma_{0} \otimes \sigma_{0}=x_{2}(z) \text { and } s_{0} \otimes s_{1}=\sigma_{0} \otimes \sigma_{0} \otimes \sigma_{0} \otimes \sigma_{z}=x_{4}(z) .
$$


Similarly, with $s_{2}$ in place of $s_{1}$, we have $x_{2}(x)$ and $x_{4}(x)$ in $Q_{2} \odot Q_{2}$.

As noted above, $s_{3} s_{4}=-i \sigma_{x} \otimes \sigma_{0}$. Similarly,

$$
s_{3} s_{5}=i \sigma_{y} \otimes \sigma_{o} \text { and } s_{4} s_{5}=-i \sigma_{z} \otimes \sigma_{o} .
$$

Hence,

$$
\left(s_{3} \otimes s_{4}\right)\left(s_{4} \otimes s_{4}\right)=s_{3} s_{4} \otimes s_{4} s_{4}=-i \sigma_{y} \otimes \sigma_{0} \otimes \sigma_{0} \otimes \sigma_{0}=-i x_{1}(y)
$$

and similarly

$$
\left(\left(s_{3} \otimes s_{5}\right)\left(s_{5} \otimes s_{5}\right)=i x_{1}(y) \text { and }\left(s_{4} \otimes s_{5}\right)\left(s_{5} \otimes s_{5}\right)=-i x_{1}(z) .\right.
$$

Thus, we have $x_{1}(a)$ for all $a$. In an entirely similar way, we find that considering $\left(s_{3} \otimes s_{3}\right)\left(s_{3} \otimes s_{4}\right)=-i x_{3}(y),\left(s_{3} \otimes s_{3}\right)\left(s_{3} \otimes s_{5}\right)=i x_{3}(x),\left(s_{4} \otimes s_{4}\right)\left(s_{4} \otimes s_{5}\right)=-i x_{3}(z)$. So we have $x_{3}(a) \in\left(Q_{2} \odot Q_{2}\right)^{2}$ for all $a$.

It remains to obtain $x_{2}(y)$ and $x_{4}(y)$. But now we have

$$
\left(s_{3} \otimes s_{0}\right) x_{1}(z)=\left(\sigma_{z} \otimes \sigma_{y} \sigma_{0} \otimes \sigma_{0}\right)\left(\sigma_{z} \otimes \sigma_{0} \otimes \sigma_{0} \otimes \sigma_{0}\right)=x_{2}(y)
$$

and similarly

$$
x_{3}(z)\left(s_{0} \otimes s_{3}\right)=x_{3}(y) .
$$

Hence, $x_{2}(y)$ and $x_{4}(y)$ also belong to $\left(Q_{2} \odot Q_{2}\right)^{2}$, completing the proof.

An Involution We now wish to find an involution on $M_{16}(\mathbb{C})$ fixing $Q_{2} \odot Q_{2}$ pointwise. If $\phi: \mathbf{M} \rightarrow \mathbf{M}$ is a $*$-involution on a complex $*$-algebra $\mathbf{M}$, recall that we write $\mathbf{M}_{s a}^{\phi}$ for the set of self-adjoint fixed-points of $\phi$. In finite dimensions, this is always an EJA. Suppose $\phi$ and $\psi$ are $*$-involutions on complex $*$-algebras $\mathbf{M}$ and $\mathbf{N}$, respectively: if $A$ is a Jordan subalgebra of $\mathbf{M}_{s a}^{\phi}$ and $B$ is a Jordan subalgebra of $\mathbf{N}_{s a}^{\psi}$, then it's easy to see that $A \odot B$ is a Jordan subalgebra of $(\mathbf{M} \otimes \mathbf{N})_{s a}^{\phi \otimes \psi}$.

Let $J=\left[\begin{array}{cc}0 & -\mathbf{1} \\ \mathbf{1} & \mathbf{0}\end{array}\right]$. Then

$$
\phi(a):=-J a^{T} J=-(J a J)^{T}
$$

is a $*$-involution (a $*$-antiautomorphism of period two) on $M_{n}(\mathbb{C})$. This fixes $Q_{2}$ pointwise, i.e., $\phi(\pi(a))=\pi(a)$ for every $a \in M_{2}(\mathbb{H})_{s a}$. Identifying $M_{16}(\mathbb{C})$ with $M_{4}\left(M_{4}(\mathbb{C})\right)=M_{4}(\mathbb{C}) \otimes M_{4}(\mathbb{C})$, we then have an involution

$$
\Phi=\phi \otimes \phi: M_{16}(\mathbb{C}) \rightarrow M_{16}(\mathbb{C}) .
$$

By the comments above, we have

Lemma B.3. $\Phi=\phi \otimes \phi$ fixes every element of $Q_{2} \odot Q_{2}$.

This completes the proof of Proposition B.1. Moreover, as a consequence of ([30], Theorem 4.4), we have

Corollary B.4. $Q_{2} \odot Q_{2}=M_{16}(\mathbb{C})^{\Phi}$.

Since $C^{*}(A) \simeq \mathbf{M} \simeq C^{*}(B)$ (as *-algebras with involution) implies $A \simeq \mathbf{M}_{s a}^{\phi} \simeq B$, and since $M_{16}(\mathbb{C}) \simeq C^{*}\left(M_{16}(\mathbb{R})_{s a}\right)$, we have

Corollary B.5. $Q_{2} \odot Q_{2} \simeq M_{16}(\mathbb{R})_{s a}=: R_{16}$. 


\section{Spin factors}

The Jordan-von Neumann-Wigner Classification Theorem [43] singles out exactly three classes of finite dimensional simple euclidean Jordan algebras: the matrix algebras $R_{n}, C_{n}, Q_{n}$, the exceptional Jordan algebra $M_{3}(\mathbb{O})_{\text {sa }}$, and one further type. The remaining type were dubbed Spin Factors by Topping in [60]. For each finite $\mathbb{N} \ni n>1$, there exists a unique spin factor of dimension $1+n$ (up to Jordan isomorphism [31]) denoted by $V_{n}$. Abstractly, $V_{n}$ is generated as a Jordan algebra by a spin system of cardinality $n$ : a collection of $2 \leq n \in \mathbb{N}$ symmetries (i.e. self-adjoint unitaries) $s_{p}$ in a unital JB algebra $\mathcal{A}$, with $s_{p} \neq \pm u_{\mathcal{A}}$ such that $s_{p} \bullet s_{q}=u_{\mathcal{A}} \delta_{p, q}$. It follows that $V_{n} \cong \mathbb{R} \oplus \mathbb{R}^{n}$ as a real inner product space, and also as a euclidean Jordan algebra with

$$
\left(\lambda_{0} \oplus \vec{\lambda}\right) \bullet\left(\mu_{0} \oplus \vec{\mu}\right)=\lambda_{0} \mu_{0}+\langle\vec{\lambda}, \vec{\mu}\rangle \oplus \lambda_{0} \vec{\mu}+\mu_{0} \vec{\lambda}
$$

Concretely, the usual complex Pauli matrices can be used to define the spin factors. We recall the usual complex Pauli matrices as follows

$$
u_{\mathbb{C}_{2}}=\sigma_{0}=\left(\begin{array}{ll}
1 & 0 \\
0 & 1
\end{array}\right) \quad \sigma_{1}=\left(\begin{array}{rr}
1 & 0 \\
0 & -1
\end{array}\right) \quad \sigma_{2}=\left(\begin{array}{ll}
0 & 1 \\
1 & 0
\end{array}\right) \quad \sigma_{3}=\left(\begin{array}{rr}
0 & -i \\
i & 0
\end{array}\right) .
$$

Following [31], we define for each finite $\mathbb{N} \ni n>1$ and $\forall 1 \leq p \leq n$, with $\lfloor\cdot\rfloor$ and $\lceil\cdot\rceil$ the usual floor and ceiling functions

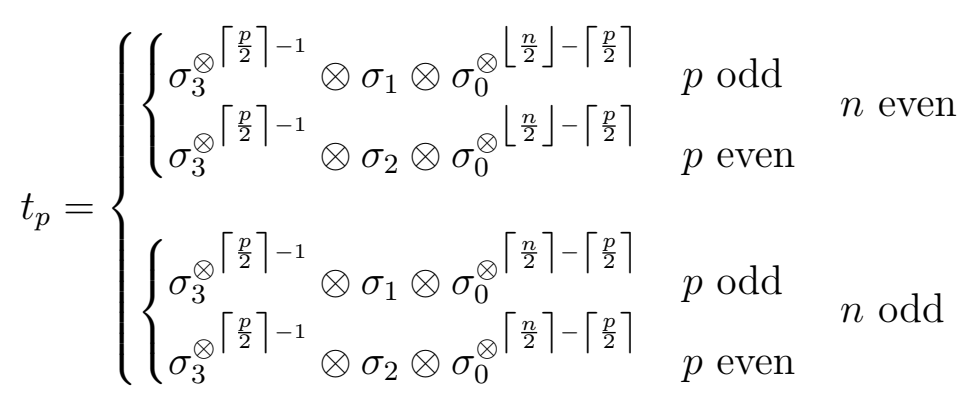

where our notation is such that $x^{\otimes^{0}}=1 \in \mathbb{R}, x \otimes 1=x=x^{\otimes^{1}}, x^{\otimes^{2}}=x \otimes x$, and so on. One can easily check that for each $n>1,\left\{t_{1}, \ldots, t_{n}\right\}$ generates a spin factor of dimension $1+n$ with $t_{p} \bullet t_{q}=\left(t_{p} t_{q}+t_{q} t_{p}\right) / 2$. It turns out [30], with finite $k \in \mathbb{N}$, that the maps

$$
\begin{array}{r}
\psi_{2 k}: V_{2 k} \longrightarrow M_{2^{k}}(\mathbb{C})_{\mathrm{sa}}:: s_{p} \longmapsto t_{p} \\
\psi_{2 k+1}: V_{2 k+1} \longrightarrow M_{2^{k}}(\mathbb{C})_{\mathrm{sa}} \oplus M_{2^{k}}(\mathbb{C})_{\mathrm{sa}}:: s_{p} \longmapsto t_{p}
\end{array}
$$

are precisely the canonical injections of $V_{n}$ into their universal $\mathrm{C}^{*}$-algebras (i.e. their universal representations). Our standard representation $\pi_{n}$ of $V_{n}$ differs from the universal representation when $n$ is odd. Specifically, when $n$ is even we define $v_{p}=t_{p}$, and 
when $n$ is odd we define $\forall 1 \leq p<n$

$$
\begin{aligned}
& v_{p}= \begin{cases}\left.\sigma_{3}^{\otimes}\right|^{\left\lceil\frac{p}{2}\right\rceil-1} \otimes \sigma_{1} \otimes \sigma_{0}^{\otimes}\left\lfloor^{\left\lfloor\frac{n}{2}\right\rfloor-\left\lceil\frac{p}{2}\right\rceil}\right. & p \text { odd } \\
\sigma_{3}^{\otimes}{ }^{\left.\otimes \frac{p}{2}\right\rceil-1} \otimes \sigma_{2} \otimes \sigma_{0}^{\otimes}\left\lfloor^{\left.\frac{n}{2}\right\rfloor-\left\lceil\frac{p}{2}\right\rceil}\right. & p \text { even }\end{cases} \\
& v_{n}=\sigma_{3}^{\otimes}\left\lfloor\frac{n}{2}\right\rfloor
\end{aligned}
$$

and we embed $V_{n}$ into its standard $\mathrm{C}^{*}$-algebra via the following Jordan monomorphism with $v_{p} \bullet v_{q}=\left(v_{p} v_{q}+v_{q} v_{p}\right) / 2$

$$
\pi_{n}: V_{n} \longrightarrow M_{2}(\mathbb{C})_{\mathrm{sa}}^{\otimes\left\lfloor\frac{n}{2}\right\rfloor}:: s_{p} \longmapsto v_{p}
$$

For example, the universal and standard representations for the qubit - i.e. $V_{3}$ - differ as follows

$$
\begin{array}{ll}
t_{1}=\sigma_{1} \otimes \sigma_{0} & v_{1}=\sigma_{1} \\
t_{2}=\sigma_{2} \otimes \sigma_{0} & v_{2}=\sigma_{2} \\
t_{3}=\sigma_{3} \otimes \sigma_{1} & v_{3}=\sigma_{3}
\end{array}
$$

hence the name standard representation. Incidentally, note that $V_{3} \cong C_{2}$ as a euclidean Jordan algebra. Furthermore, $V_{2} \cong R_{2}, V_{5} \cong Q_{2}$, and $V_{9} \cong M_{2}(\mathbb{O})_{\text {sa }}$; the ambient spaces for the real, quaternionic, and octonionic quantum bits.

\section{Weak Composites}

By a weak composite of EJAs $A$ and $B$, we mean an EJA $A B$ and a bilinear mapping $\pi: A \otimes B \rightarrow A B$ satisfying parts (a) and (b), but not necessarily part (c), of Definition 4.1. That is, we suspend the requirement that $A B$ be generated, as a Jordan algebra, by $\pi(A \otimes B)$. We are going to show that the Jordan subalgebra of $A B$ generated by the image of $A \otimes B$ in $A B$ also satisfies these conditions, and hence, is a composite in the strict sense.

Observe, first, that we made no use of Condition (c) in proving any of the results leading up to, and including, Corollary 4.4. So all of these are also satisfied by weak composites.

Proposition D.1. Let $A B$ be a weak composite of EJAs $A$ and B. Then the Jordan subalgebra of $A B$ generated by $A \otimes B$ is also a composite.

Proof: Identifying $A \otimes B$ with its image, $\pi(A \otimes B)$, in $A B$, let $A \odot B=J(A \otimes B)$ denote the Jordan subalgebra of $A B$ generated by $A \otimes B$. That the co-restriction $\pi: A \otimes$ $B \rightarrow A \odot B$ satisfies the conditions for a composite (Definition 2.1) is straightforward. It is also straightforward that $A \odot B$ will satisfy the conditions for a composite of Jordan models (Definition 4.1), provided that it satisfies the conditions for a dynamical 
composite (Definition 2.3). To see that it does, let $\phi \in G(A)$ and $\psi \in G(B)$. We need to show that $\phi \otimes \psi$ preserves $A \odot B$. We can expand $\phi$ as $U_{a} \circ g$ and $\psi$ as $U_{b} \circ h$ for some interior elements $a \in A_{+}, b \in B_{+}$, and Jordan homomorphisms $g \in G(A)$ and $h \in G(B)([26]$, III.5.1). Now

$$
\phi \otimes \psi=\left(U_{a} \circ g\right) \otimes\left(U_{b} \circ h\right)=\left(U_{a} \otimes U_{b}\right) \circ(g \otimes h) .
$$

Since $(g \otimes h)\left(u_{A B}\right)=g\left(u_{A}\right) \otimes h\left(u_{B}\right)=u_{A} \otimes u_{B}=u_{A B}, g \otimes h$ is a symmetry, hence, a Jordan automorphism of $A B$ ([4], Theorem 2.8). As it maps $A \otimes B$ to itself, it also preserves $J(A \otimes B)$, i.e., $A \odot B$.

It remains to show that $U_{a} \otimes U_{b}$ also preserves $A \odot B$. Begin with the observation that if $A$ is an EJA and $X \subseteq A$, then for all $x \in X, U_{x}(J(X)) \subseteq J(X)$. This is evident from the fact that, as $x \in J(X)$ and the latter is a Jordan subalgebra of $A$, $U_{x}(y)=2 x \bullet(x \bullet y)-\left(x^{2}\right) \bullet y$ for all $y \in J(X)$.

Now consider that the proof of the identities $(a \otimes u) \bullet(x \otimes y)=(a \bullet x) \otimes y$ and $(u \otimes b) \bullet(x \otimes y)=x \otimes(b \bullet y)$ relies only on (a) and (b), and so, holds in our context. By Corollary 4.4, $U_{a \otimes u}=U_{a} \otimes \mathrm{id}$ and $U_{u \otimes b}=\mathrm{id}_{A} \otimes U_{b}$. If $a, b$ are interior elements, then $U_{a} \in G(A)$ and $U_{b} \in G(B)$, so by (b), we have

$$
U_{a} \otimes U_{b}=\left(U_{a} \otimes \operatorname{id}_{B}\right) \circ\left(\operatorname{id}_{A} \otimes U_{b}\right)=U_{a \otimes u_{B}} \circ U_{u_{A} \otimes b} .
$$

Since $U_{a \otimes u_{B}}$ and $U_{u_{A} \otimes b}$ preserve $J(A \otimes B)=A \odot B$, by the remark above, so does $U_{a} \otimes U_{b}$, and the proof is finished.

\section{E Extending order automorphisms}

In this appendix, for the reader's convenience, we collect facts concerning extensions of derivations used in the body of this paper. Throughout, $A$ is an EJC algebra, that is, a Jordan subalgebra of $\mathbf{M}_{s a}$, where $\mathbf{M}$ is a finite-dimensional complex matrix algebra. We begin by recalling some facts about order-automorphisms and Jordan derivations ([4], Chapter 6).

If $\{\phi(t)\}_{t \in \mathbb{R}}$ is a one-parameter group of order-automorphisms of $A$, then for every $a \in A, \phi(t)(a)=e^{t \delta} a$ where $\delta=\phi^{\prime}(0)$ is a linear operator on $A$. The linear operators $\delta$ arising in this way are called order derivations of $A$. Order-derivations come in two basic types: those having the form $\delta=L_{a}$ for some $a \in A$ and those having the property $\delta(u)=0$. The latter are exactly the Jordan derivations of $A$, that is, the linear maps $A \rightarrow A$ satisfying the Leibniz law $\delta(a \bullet b)=\delta(a) \bullet b+a \delta(b)$ for all $a, b \in A$. Order derivations of the form $L_{a}$ are said to be self-adjoint; those that are Jordan derivations are said to be skew. The former are self-adjoint with respect to the canonical inner product on $A$, by the definition of a euclidean Jordan algebra, while the latter are skew-adjoint. Every order-derivation has the form $\delta=L_{a}+\delta^{\prime}$ where $\delta^{\prime}$ is skew ([4], Proposition 1.60). It follows that $\phi:=e^{t \delta}$ fixes the Jordan unit $u_{A}$ iff $\delta$ is skew.

A mapping $\delta: \mathbf{M} \rightarrow \mathbf{M}$ is said to be an order-derivation of $\mathbf{M}$ iff it preserves $\mathbf{M}_{s a}$ and its restriction to $\mathbf{M}_{s a}$ is an order derivation in the sense discussed above. If $a \in \mathbf{M}$, 
define $\delta_{a}: \mathbf{M} \rightarrow \mathbf{M}$ by

$$
\delta_{a}(x)=\frac{1}{2}\left(a x+x a^{*}\right) .
$$

Then $\delta_{a}$ is an order derivation in this broader sense. Moreover, every order-derivation of $\mathbf{M}$ has this form for some $a \in \mathbf{M}$ ([4], Appendix 183.) Note that $\delta_{a}$ is self-adjoint precisely when $a$ is self-adjoint and skew precisely when $a$ is skew-adjoint. In the latter case, a direct computation shows that $\delta_{a}$ is actually a $*$-derivation of $\mathbf{M}$, that is, $\delta_{a}(x y)=\delta_{a}(x) y+x \delta_{a}(y)$ and $\delta_{a}\left(x^{*}\right)=\delta_{a}(x)^{*}$.

The following essentially restates Lemma 5.3:

Lemma E.1. Any Jordan derivation of $A$ extends to a $*$-derivation of $\mathbf{M}$. Hence, any order-derivation of $A$ extends to an order-derivation of $\mathbf{M}$.

Proof. The set of derivations of a finite-dimensional real or complex algebra is a Lie algebra, and in the case of a Jordan algebra $A$, it is the linear span of the elements $\left[L_{a}, L_{b}\right]$, for $a, b \in A$, in fact every derivation is a sum of such elements. ${ }^{30}$ Since $L_{a}$ and $L_{b}$ are linear operators $A \rightarrow A,\left[L_{a}, L_{b}\right]$ belongs to the real associative algebra of such operators. Since $A$ is a subalgebra of $\left(\mathbf{M}_{A}\right)_{s a}, L_{a}$ and $L_{b}$ extend to linear operators $\left(\mathbf{M}_{A}\right)_{s a} \rightarrow\left(\mathbf{M}_{A}\right)_{s a}$, namely the Jordan multiplication operators corresponding to $a$ and $b$ viewed as elements of $\left(\mathbf{M}_{A}\right)_{s a}$. But as noted above, every Jordan derivation of $\mathbf{M}_{A}$ is also a $*$-derivation for the associative product, establishing the first claim. A *-derivation on $\mathbf{M}$ preserves $\mathbf{M}_{s a}$, and is also a Jordanderivation on the latter, so this also establishes that all skew order derivations on $A$ extend to order-derivations of $\mathbf{M}$. A self-adjoint order derivation of $A$ is simply a map $L_{a}: A \rightarrow A$ with $a \in A$, which extends to $\delta_{a}: \mathbf{M} \rightarrow \mathbf{M}$ since $a$ is self-adjoint in $\mathbf{M}$. Thus, all order-derivations on $A$ extend to order-derivations on $\mathbf{M}$.

Lemma E.2. Any one-parameter group of order automorphisms of A extends to a one-parameter group of order-automorphisms of $\mathbf{M}$.

Proof: If $\{\phi(t)\}_{t \in \mathbb{R}}$ is a one-parameter group of order-automorphisms of $A$, then $\phi(t)(a)=$ $e^{t \delta} a$ where $\delta=\phi^{\prime}(0)$ is an order-derivation of $A$. Thus, we have $\delta=L_{a}+\delta^{\prime}$ where $a \in A$ and $\delta^{\prime}$ is skew ([4], Proposition 1.60). $L_{a}$ obviously extends from $A$ to $\mathbf{M}$, simply because $a \in \mathbf{M}$ and the Jordan product on $A$ is the restriction of that on $\mathbf{M}$. By Lemma 5.3, $\delta^{\prime}$ also extends to a Jordan derivation $\delta^{\prime \prime}$ on $\mathbf{M}$. Thus, we have an extension $\widehat{\delta}=L_{a}+\delta^{\prime \prime}$ on $\mathbf{M}$. In particular, $\delta^{\prime \prime}(A) \subseteq A$. We now have an order-automorphism $\widehat{\phi}(t)=e^{t \widehat{\delta}}$ of $\mathbf{M}_{+}$. Note that this preserves $A$, since

$$
\widehat{\phi}(t) x=\sum_{k=1}^{\infty} \frac{t^{k}}{k !} \widehat{\delta}^{k} x
$$

and $\widehat{\delta} x=\left(L_{a}+\delta^{\prime \prime}\right) x=a \bullet x+\delta^{\prime \prime}(x)$, which belongs to $A$ if $x$ does.

\footnotetext{
${ }^{30}$ See e.g. [26], Proposition II.4.1 for the fact that these are derivations, called inner derivations. In [61], for example, it is said to be well-known that all derivations of a finite-dimensional JB algebra (i.e. an EJA) are inner.
} 
Corollary E.3. Every element of $G(A)$ extends to an element of $G\left(\left(\mathbf{M}_{A}\right)_{\text {sa }}\right)$.

Lemma E.4. Let $A$ and $B$ be EJC-algebras. If $\delta$ is any *-derivation of $\mathbf{M}_{A}$ fixing $A$, then $\delta \otimes \mathbf{1}$ is a *-derivation of $\mathbf{M}_{A} \otimes \mathbf{M}_{B}$ fixing $A \odot B$.

Proof: Let $\mathbf{M}$ and $\mathbf{N}$ be $*$-algebras, and let $a, b \in \mathbf{M}$ and $x, y \in \mathbf{N}$. Then

$$
(a \otimes x) \bullet(b \otimes y)=\frac{1}{2}(a b \otimes x y+b a \otimes y x) .
$$

If $\delta$ is a $*$-derivation of $\mathbf{M}$, then it is straighforward to check that $\delta \otimes \mathbf{1}$ is a $*$-derivation of $\mathbf{M} \otimes \mathbf{N}$, and that for all $a, b \in \mathbf{M}$ and $x, y \in \mathbf{N}$,

$$
(\delta \otimes \mathbf{1})((a \otimes x) \bullet(b \otimes y))=(a \otimes x) \bullet(\delta(b) \otimes y)+(\delta(a) \otimes x) \bullet(b \otimes y) .
$$

In particular, if $A \subseteq \mathbf{M}$ and $\delta(A) \subseteq A$, it follows that $(\delta \otimes \mathbf{1})(A \otimes B) \subseteq(A \otimes B) \bullet(A \otimes B)$ for any $B \subseteq \mathbf{N}$. It follows easily that, where $A$ and $B$ are EJCs and $\mathbf{M}=\mathbf{M}_{A}$ and $\mathbf{N}=\mathbf{M}_{B}, \delta \otimes \mathbf{1}$ preserves $A \odot B .{ }^{31}$

Proposition E.5. If $\phi$ and $\psi$ are order-automorphisms in $G(A)$ and $G(B)$, respectively, then $\phi \otimes \psi: A \otimes B \rightarrow A \otimes B$ extends to an order-automorphism in $G(A \odot B)$.

Proof: By Corollary E.3, we can assume that $\phi$ is an order-automorphism of $M_{s a}$ fixing $A$. Since $\phi \in G(A)$, it occurs as part of a one-parameter group $\phi(t)=e^{t \delta}$ of orderautomorphisms, say as $\phi=\phi(1)=e^{\delta}$, where $\delta$ is an order-derivation of $A$. By Lemma E.1, $\delta$ extends to an order derivation of $\mathbf{M}$ fixing $A$. It follows that

$$
\phi \otimes \mathbf{1}=e^{t \delta} \otimes \mathbf{1}=\sum_{n=0}^{\infty} \frac{t^{n}}{n !} \delta^{n} \otimes \mathbf{1}=\sum_{n=0}^{\infty} \frac{t^{n}}{n !}(\delta \otimes \mathbf{1})^{n}=e^{t(\delta \otimes \mathbf{1})} .
$$

By Lemma E.4, $\delta \otimes \mathbf{1}$ fixes $A \odot B$; thus, so does the series at right, whence, so does $\phi \otimes 1$. It follows that if $\phi$ is an order-automorphism of $\left(M_{A}\right)_{s a}$ fixing $A$, so $\phi \otimes \mathbf{1}$ is an order-automorphism of $A \otimes B$ fixing $J(A \otimes B)=A \odot B$. Hence, if $\phi$ and $\psi$ are orderautomorphisms of $M_{A}$ and $M_{B}$, respectively fixing $A$ and $B$, then $\phi \otimes \psi=(\phi \otimes \mathbf{1}) \circ(\mathbf{1} \otimes \psi)$ fixes $A \odot B$.

\footnotetext{
${ }^{31}$ The details: let $\delta$ be a $*$-derivation on a $*$-algebra $\mathbf{M}$, and let $X \subseteq M_{s a}$ with $\delta(X) \subseteq X$. Let $Y=\{a \in J(X) \mid \delta(a) \in J(X)\}$. Evidently $X \subseteq Y$. Now if $a, b \in Y$ and $t \in \mathbb{R}$, then $\delta(t a+b)=$ $t \delta(a)+\delta(b) \in J(X)$, so $J(X)$ is a subspace of $M$. If $a, b \in Y$ then $\delta(a \bullet b)=a \bullet \delta(b)+\delta(a) \bullet b \in J(X)$. Thus, $Y$ is a Jordan subalgebra of $\mathbf{M}_{s a}$, containing $X$, and contained in $J(X)$. Ergo, $Y=J(X)$, and $\delta(J(X)) \subseteq J(X)$.
} 